JOURNAL OF THE

AMERICAN MATHEMATICAL SOCIETY

Volume 18, Number 1, Pages 61-120

S 0894-0347(04)00470-9

Article electronically published on October 14, 2004

\title{
MULTIDIMENSIONAL VISCOUS SHOCKS I: DEGENERATE SYMMETRIZERS AND LONG TIME STABILITY
}

\author{
OLIVIER GUÈS, GUY MÉTIVIER, MARK WILLIAMS, AND KEVIN ZUMBRUN
}

\section{Contents}

Part 1. Introduction 62

1. Guide to the paper $\quad 62$

2. Assumptions 67

3. Auxiliary assumption on the shock 72

4. Main results 74

Part 2. Algebraic preparation 75

5. Doubling and conjugation 75

6. Block structure and standard symmetrizers 77

7. Evans function for the doubled boundary problem

Part 3. Zero mass perturbations 89

8. Degenerate symmetrizer for small frequencies $\quad 89$

9. Large frequency estimate 92

10. Linear and nonlinear stability 93

Part 4. Nonzero mass perturbations $\quad 100$

11. Nonlinear stability 100

12. $L^{1}-L^{p}$ estimates, $p \geq 2 \quad 102$

13. Nonlinear stability for $d=2 \quad 112$

References 117

Received by the editors September 18, 2002.

2000 Mathematics Subject Classification. Primary 35L60; Secondary 35B35, 35B45, 35K55, 35L65, 76L05.

Key words and phrases. Viscous shock stability, Evans-Lopatinski condition, Kreiss symmetrizers.

Research was supported in part by European network HYKE grants HPRN-CT-2002-00282 (O.G.) and HPRN-CT-2002-00282 (G.M.) and by NSF grants DMS-0070684 (M.W.) and DMS0070765 (K.Z.).

(C)2004 American Mathematical Society Reverts to public domain 28 years from publication 


\section{Part 1. Introduction}

In this paper we study the long time stability of multidimensional planar viscous shocks with energy estimates. We introduce degenerate symmetrizers as the main new tool for proving the estimates.

\section{Guide to the PAPER}

1.1. Ideal shocks and viscous shocks. Consider a system of conservation laws

$$
u_{t}+\sum_{j=1}^{d} f^{j}(u)_{x_{j}}=0,
$$

where $u, f^{j} \in \mathbb{R}^{n}$, and a planar (ideal) shock $\left(U_{R}, U_{L}, s\right)$ moving in the $x_{1}$ direction with speed $s$. This means that the triple $\left(U_{R}, U_{L}, s\right)$ is constant and satisfies

$$
s[U]-\left[f^{1}(U)\right]=0,
$$

where $[U]=U_{R}-U_{L}$. Condition (1.2), known as the Rankine-Hugoniot condition, is necessary and sufficient for the function $u$ defined by

$$
u= \begin{cases}U_{R}, & x_{1}>s t, \\ U_{L}, & x_{1}<s t\end{cases}
$$

to be a weak solution of (1.1) in $\mathbb{R}_{t, x}^{d+1}$.

Redefining $x_{1}, f^{1}$ as $\tilde{x_{1}}=x_{1}-s t$ and $\tilde{f}^{1}(u)=f^{1}(u)-s u$, we can and will henceforth assume $s=0$.

Consider also a corresponding system of viscous conservation laws

$$
u_{t}+\sum_{j=1}^{d} f^{j}(u)_{x_{j}}=\triangle u,
$$

where

$$
\triangle u=\sum_{j=1}^{d} \partial_{x_{j}}^{2} u,
$$

and a steady state solution $\psi\left(x_{1}\right)$ connecting the endstates $U_{R}, U_{L}$ :

$$
\lim _{x_{1} \rightarrow+\infty} \psi\left(x_{1}\right)=U_{R}, \quad \lim _{x_{1} \rightarrow-\infty} \psi\left(x_{1}\right)=U_{L} .
$$

Note that $\psi$ satisfies the travelling wave ODE

$$
\psi^{\prime}=f^{1}(\psi)-f^{1}\left(U_{L}\right)
$$

It is easy to check that the Rankine-Hugoniot condition is a necessary condition for the existence of such a $\psi$. We refer to $\psi$ variously as a connection, a profile, and a viscous shock.

1.2. Nonlinear stability. We wish to understand the stability of the profile $\psi\left(x_{1}\right)$ under multidimensional perturbations. Let $\mathcal{A}$ denote some set of admissible perturbations to be specified later. 
Definition 1.1. For $v_{0} \in \mathcal{A}$ let $u(x, t)$ be the solution to the system (1.4) with initial data at $t=0$ given by

$$
u_{0}(x)=\psi\left(x_{1}\right)+\epsilon v_{0}(x) .
$$

We say that $\psi$ is nonlinearly stable with respect to perturbations in $\mathcal{A}$ if there exists an $\epsilon_{0}>0$ (depending on $\left|v_{0}\right|_{\mathcal{A}}$ ) such that for $\epsilon \leq \epsilon_{0}$, the solution $u(x, t)$ exists for all time and

$$
\left|u(x, t)-\psi\left(x_{1}\right)\right|_{L^{\infty}(x)} \rightarrow 0 \text { as } t \rightarrow \infty .
$$

1.3. The eigenvalue equation. Let $A_{j}\left(x_{1}\right)=d f^{j}\left(\psi\left(x_{1}\right)\right)$ and $x^{\prime}=\left(x_{2}, \ldots, x_{d}\right)$. (Later, we will switch to a more convenient $(x, y)=\left(x_{1}, x^{\prime}\right)$ notation.) After a transfer of initial data to forcing carried out in Section 2, the key step turns out to be the proof of good estimates for the linear problem

$$
\begin{aligned}
& \text { (a) } u_{t}+\left(A\left(x_{1}\right) u\right)_{x_{1}}+\sum_{j=2}^{d} A_{j}\left(x_{1}\right) \partial_{j} u-\triangle u=f, \\
& \text { (b) }\left.u\right|_{t=0}=0 .
\end{aligned}
$$

Because of the initial condition, if we extend $u$ and $f$ by zero into $t<0$, the extensions satisfy (1.9) on $\mathbb{R}^{1+d}$. We may take the Fourier transform in $\left(t, x^{\prime}\right)$ to obtain the eigenvalue equation for $\hat{u}\left(x_{1}, \lambda, \xi^{\prime}\right)$ :

$$
\hat{u}_{x_{1} x_{1}}-\left(A_{1}\left(x_{1}\right) \hat{u}\right)_{x_{1}}-s\left(x_{1}, \lambda, \xi^{\prime}\right) \hat{u}=\hat{f}\left(x_{1}, \lambda, \xi^{\prime}\right)
$$

where $\left(\tau, \xi^{\prime}\right)$ is dual to $\left(t, x^{\prime}\right), \lambda=i \tau+\gamma$ with $\gamma \geq 0$ and

$$
s\left(x_{1}, \lambda, \xi^{\prime}\right)=\sum_{j=2}^{d} A_{j}\left(x_{1}\right) i \xi_{j}+\lambda I+\left|\xi^{\prime}\right|^{2} I .
$$

The existence of "eigenvalues" $\lambda$ in the unstable half-space $\Re \lambda>0$, that is, values of $\lambda$ for which there exist nontrivial solutions $\hat{u}\left(x_{1}, \lambda, \xi^{\prime}\right)$ of (1.10) (with $\hat{f}=0$ ) decaying at both $\pm \infty$, is easily seen to rule out any useful stability estimate for (1.9) (see Remark 2.4). In Section 2 we recall the definition of the Evans function $\mathcal{D}\left(\lambda, \xi^{\prime}\right)$ corresponding to the viscous profile $\psi$. This function is a Wronskian of solutions to the homogeneous version of 11.10 with the property that eigenvalues of (1.10) in $\Re \lambda>0$ correspond to zeros of $\mathcal{D}\left(\lambda, \xi^{\prime}\right)$.

It is easy to check that $\psi^{\prime}\left(x_{1}\right)$ itself is a solution of the eigenvalue equation when $\left(\lambda, \xi^{\prime}\right)=(0,0)$ (differentiate (1.6) twice), so the Evans function (suitably extended) vanishes at $\left(\lambda, \xi^{\prime}\right)=(0,0)$. One of the main hypotheses of this paper is that $\mathcal{D}$ vanishes to precisely first order at $(0,0)$ and has no other zeros in $\Re \lambda \geq 0$. This is stated precisely as assumption (H4) in Section 2.

The same Evans assumption has already been shown to imply long time stability of viscous profiles in the $1 \mathrm{D}$ case in $[\mathrm{KK}]$ for zero mass perturbations and [Z2, MaZ1, MaZ2, MaZ3, MaZ4, MaZ5, Z3, HZ, Ra for general perturbations, and in the multidimensional case in [Z1 [Z3, Z4] (for general perturbations); see also the important groundwork of [GZ, ZH, ZS] and [K1, K2, KS, LZe, [H1, H2]. A treatment of the scalar multidimensional case (for which the Evans assumption always holds, by the maximum principle) may be found in HoZ2, HoZ3. We say more about the relation of [KK] and [Z1] to this work below.

We also mention the earlier works [Go1, Go2, MN, KMN] in which stability under zero mass perturbations was proved for sufficiently weak (i.e., small amplitude) 
shocks in 1D, $[\mathrm{SX}$ in which stability under general perturbations was proved for weak shocks in 1D, and [Go3 GM] in which the stability of weak planar shock solutions for viscous scalar multidimensional conservation laws was demonstrated. See also the important partial results of [L1] and [L2] for weak shocks in 1D, in which the modern picture of $1 \mathrm{D}$ asymptotic behavior of a perturbed shock profile (verified for strong shocks in Ra ) was first set out, and the treatment of special nonLax shocks in [LX, FL, LZ1, LZ2] (extended to general strong shocks in [HZ, Ra]). There is little contact either in techniques or ideas between the 1D weak shock theory and our present analysis, which is closer to the inviscid theory of Kreiss and Majda K, M1, M2. Our treatment of stability in 2D has some conceptual roots in [Go3, GM], as described further in [Z1], but at a technical level it is again essentially unrelated.

Remark 1.1. Recent work by Freistühler and Szmolyan [FS] and independently by Plaza and Zumbrun [PZ] shows that Evans condition (H4) holds for sufficiently small amplitude Lax shocks, under the mild structural assumptions of symmetrizability plus strict concavity/convexity of the characteristic associated with the shock; see [FM, Met1, [Met2] for the analogous study of small-amplitude inviscid stability. In general, (H4) may fail for large-amplitude or non-Lax shocks; see [ZS, GZ, FZ].

1.4. Reformulation as a doubled boundary problem. Rewrite the second order $n \times n$ eigenvalue equation as a $2 n \times 2 n$ first order system on $\mathbb{R}_{x_{1}}$ depending on frequency $\left(\lambda, \xi^{\prime}\right)$ :

$$
\left(\begin{array}{l}
\hat{u} \\
\hat{v}
\end{array}\right)_{x}=\left(\begin{array}{cc}
A_{1}\left(x_{1}\right) & I \\
s\left(x_{1}, \lambda, \xi^{\prime}\right) & 0
\end{array}\right)\left(\begin{array}{l}
\hat{u} \\
\hat{v}
\end{array}\right)+\left(\begin{array}{c}
0 \\
\hat{f}\left(x_{1}, \lambda, \xi^{\prime}\right)
\end{array}\right)
$$

or

$$
\mathcal{U}_{x_{1}}=\mathcal{G U}+\mathcal{F}
$$

for short.

Notation 1.1. If $f\left(x_{1}\right)$ is any function defined on $\mathbb{R}$, define $f_{ \pm}$for $x_{1} \geq 0$ by

$$
\begin{aligned}
& f_{+}\left(x_{1}\right)=f\left(x_{1}\right), \\
& f_{-}\left(x_{1}\right)=f\left(-x_{1}\right) .
\end{aligned}
$$

Now on $x_{1} \geq 0$ let

$$
\begin{aligned}
& U\left(x, \lambda, \xi^{\prime}\right)=\left(\mathcal{U}_{+}, \mathcal{U}_{-}\right), \\
& G\left(x, \lambda, \xi^{\prime}\right)=\left(\begin{array}{cc}
\mathcal{G}_{+} & 0 \\
0 & -\mathcal{G}_{-}
\end{array}\right), \\
& F=\left(\begin{array}{c}
\mathcal{F}_{+} \\
-\mathcal{F}_{-}
\end{array}\right), \text {and } \\
& \Gamma U=\mathcal{U}_{+}-\mathcal{U}_{-} .
\end{aligned}
$$

Note that $\mathcal{U}\left(x_{1}, \lambda, \xi^{\prime}\right)$ satisfies (1.12) on $\mathbb{R}$ if and only if $U$ satisfies the $4 n \times 4 n$ first order system on $x_{1} \geq 0$ :

$$
\begin{aligned}
& U_{x_{1}}-G\left(x_{1}, \lambda, \xi^{\prime}\right) U=F \text { on } x_{1} \geq 0, \\
& \Gamma U=0 \text { on } x_{1}=0 .
\end{aligned}
$$


The boundary condition in (1.15) just expresses the continuity of $\mathcal{U}$ (in (1.12) ) at $x_{1}=0$. Usually, we will drop the script notation and write $U=\left(U_{+}, U_{-}\right)$.

Having rewritten the eigenvalue equation as a boundary problem on half-space, we are now in a position to use the machinery of Kreiss symmetrizers to prove energy estimates. But the most serious obstacle remains, namely, the dependence of $G$ on $x_{1}$. Trouble is caused, for example, by the fact that as $x_{1}$ varies from $-\infty$ to $+\infty$, one of the eigenvalues of $A_{1}\left(x_{1}\right)$ changes sign (the $k$ th eigenvalue if the inviscid shock is a $k$ shock).

1.5. Conjugation. To deal with the $x_{1}$ dependence, we use a tool introduced in MZ1. Observe that since the $x_{1}$ dependence of $G$ enters only through the profile $\psi\left(x_{1}\right)$, there is a well-defined limiting problem corresponding to (1.15) which is obtained simply by letting $x_{1} \rightarrow+\infty$ in $G$. Call the limit matrix $G\left(\infty, \lambda, \xi^{\prime}\right)$. One can replace (1.15) with a constant coefficient problem by constructing a matrix $W\left(x_{1}, \lambda, \xi^{\prime}\right)$ with the properties

$$
\begin{aligned}
& W\left(x_{1}, \lambda, \xi^{\prime}\right)=I+O\left(e^{-\theta x_{1}}\right), \quad \theta>0, \\
& \partial_{x_{1}} W=G\left(x_{1}\right) W\left(x_{1}\right)-W\left(x_{1}\right) G(\infty) .
\end{aligned}
$$

The substitution $U=W V$ then transforms the problem (1.15) into

$$
\begin{aligned}
& V_{x_{1}}-G\left(\infty, \lambda, \xi^{\prime}\right) V=W^{-1} F, \\
& \tilde{\Gamma}\left(x_{1}, \lambda, \xi^{\prime}\right) V=0 \text { on } x_{1}=0,
\end{aligned}
$$

where $\tilde{\Gamma}\left(x_{1}, \lambda, \xi^{\prime}\right) V=\Gamma W\left(x_{1}, \lambda, \xi^{\prime}\right) V$. Thus, estimates for 1.17) imply estimates for 1.15 .

1.6. Degenerate symmetrizers. Kreiss symmetrizers have long $[\mathrm{K}]$ been used to obtain $L^{2}$ estimates for hyperbolic boundary problems with nonvanishing Lopatinski determinants. In MZ1 the use of such symmetrizers was extended to the Dirichlet problem for "hyperbolic + viscosity" operators and applied to study the stability of multidimensional viscous Dirichlet boundary layers, a situation where the Lopatinski determinant is nonvanishing. Standard symmetrizers adapted to hyperbolic-parabolic operators are recalled in Section 5.

The linear algebraic preparation needed for the construction of both standard and degenerate symmetrizers is given in Part 2.

As we have seen, the Evans function in the viscous shock problem vanishes for zero frequency. When the eigenvalue problem on the whole space is reformulated as a doubled boundary problem on a half space, the vanishing of the Evans function translates into vanishing of the Lopatinski determinant for the boundary problem (1.17).

In Section 7 of this paper we construct degenerate symmetrizers to cope with the degeneracy of the Lopatinski determinant in the viscous shock problem. The Lopatinski determinant is nonvanishing for frequencies bounded away from zero, so most of our efforts are focused on the small frequency region. The critical estimate for (1.17) is the small frequency estimate (8.12), where the norm is an $L^{2}\left(x_{1}\right)$ norm, the functions $U, F$ depend on $\left(x_{1}, \lambda, \xi^{\prime}\right)$, and $\rho=\left|\lambda, \xi^{\prime}\right|$. Note that the estimate is quite singular at $\rho=0$. 
1.7. Zero mass perturbations. Our first application of this estimate is a proof of the long time stability of multidimensional planar shocks under zero mass perturbations in dimensions $\geq 3$. This means we take $v_{0}(x)$ in (1.7) of the form

$$
v_{0}=\operatorname{div} V
$$

for sufficiently well-behaved $V$ (see assumption (H5)).

There is no restriction on the size of the shocks, but they are required to satisfy the Evans assumption (H4). To deal with the singularity at $\rho=0$ in the main estimate (8.12), we are led to introduce mixed norms corresponding to the space $L^{2}\left(x_{1}, L^{1}\left(t, x^{\prime}\right)\right)\left(x_{1}=0\right.$ is the boundary). We are then able to carry out in higher dimensions the strategy used in $\mathrm{KK}$ for handling zero mass perturbations in 1D, with our mixed norm playing the role of their $L^{1}$ norm. The strategy uses the zero mass assumption to write the forcing in divergence form (Remark 2.2). First one solves an auxiliary problem (10.3) and then reduces to considering a problem with $\rho F$ forcing (10.5). Clearly, such forcing is advantageous in the region where $\rho$ is small. A more detailed description of this strategy is given at the beginning of Section 9. The full linear stability estimate for (1.9) in this case is (taking $f=\operatorname{div} \mathcal{F})$

$$
\left|u, u_{t}, u_{x^{\prime}}\right|_{L^{2}(t, x)}+\left|u_{x_{1}}\right|_{L^{2}(t, x)} \leq C\left(|\mathcal{F}|_{L^{2}\left(x_{1}, L^{1}\left(t, x^{\prime}\right)\right)}+|\mathcal{F}|_{L^{2}(t, x)}\right) .
$$

This is (10.1). The zero mass result is Theorem 4.1

1.8. Nonzero mass perturbations. Let $\left|\tau, \xi^{\prime}\right|=r \leq \rho$. Since Lebesgue measure in $d$-dimensional $\left(\tau, \xi^{\prime}\right)$ space is

$$
d \tau d \xi^{\prime}=r^{d-1} d r d \omega
$$

the mixed norm argument used to prove (8.22) works more easily in higher dimensions: $r^{d-1}$ cancels the singularity at $\rho=0$ in the main estimate (8.12). In fact, for $d \geq 5$ more general perturbations not in divergence form can be handled by an argument that is simpler than the one just described. There is no need to consider an auxiliary problem. In place of (1.19) the estimate for (1.9) is now

$$
\left|u, u_{t}, u_{x^{\prime}}\right|_{L^{2}(t, x)}+\left|u_{x_{1}}\right|_{L^{2}(t, x)} \leq C\left(|f|_{L^{2}\left(x_{1}, L^{1}\left(t, x^{\prime}\right)\right)}+|f|_{L^{2}(t, x)}\right) .
$$

Observe that there is a gain of two derivatives in (1.19) but a gain of only one in (1.20). The result for nonzero mass perturbations in $d \geq 5$ is Theorem 4.3 .

1.9. $L^{1}-L^{p}$ estimates. Of course, one wants to prove nonlinear stability for nonzero mass perturbations in all dimensions $d \geq 2$. However, the small frequency estimate of Part 3, (8.12), does not appear adequate to handle the problem of long time stability under nonzero mass perturbations in space dimensions 2,3 , and 4 . Thus, we are led in Part 3 to prove more refined $L^{1}-L^{p}$ estimates $(p \geq 2)$ by a combination of degenerate symmetrizer and duality arguments. These are crucial for the treatment of long time stability, and as far as we know are the first such multidimensional symmetrizer estimates between norms other than $L^{2}$. Previous attempts had been foiled by the presence of glancing modes. Indeed, such modes present the main obstacle to be overcome in Section 11. The bounds we obtain by our symmetrizer estimates match those obtained by direct integration of the pointwise resolvent kernel bounds obtained by explicit computation in [Z1]. 
In Section 11 we define an adjoint doubled boundary problem dual to the original forward problem and observe that $L^{2}-L^{\infty}$ estimates for the dual problem are equivalent to $L^{1}-L^{2}$ estimates for the forward problem. Section 11 is devoted to the proof of $L^{2}-L^{\infty}$ estimates for the dual problem. In order to obtain these estimates we must add the structural assumption (H6) of [Z1] that the glancing set associated with the shock have constant rank; see our (H6) below.

In place of 1.20 we obtain for nonzero mass perturbations in dimensions $d \geq 3$ :

$$
\left|u, u_{t}, u_{x^{\prime}}\right|_{L^{2}(t, x)}+\left|u_{x_{1}}\right|_{L^{2}(t, x)} \leq C\left(|f|_{L^{1}(t, x)}+|f|_{L^{2}(t, x)}\right) .
$$

The corresponding nonlinear stability result in $d \geq 3$ is Theorem 4.4

The passage from the linear stability estimates (1.19), (1.20), (1.21) to the corresponding nonlinear stability results in Theorems 4.1, 4.3, and 4.4 is in each case by an argument similar to the scheme in [KK]. These arguments are given in Sections 9 and 10.

The endgame in dimension two seems to require a special argument similar to the one in [Z1]. This is given in Section 12. The corresponding nonlinear stability result is Theorem 4.5. Here, the inverse Laplace transform is estimated on a parabolic contour $\Re \lambda=-C^{-1}\left(\left|\xi^{\prime}\right|^{2}+|\Im \lambda|^{2}\right)$, rather than the flat contour $\Re \lambda=0$ considered in KK], to take into account the additional decay due to diffusion in the parabolic case. By contrast, the $\mathrm{KK}$ endgame takes into account (and requires) only estimates like those in the hyperbolic case, which for general (nonzero mass) perturbations are sufficient in large enough dimension, but fail in dimensions one and two.

1.10. Assessment. The stability of multidimensional planar shocks has already been carefully studied in [Z1] by construction of Green's functions. [Z1] proves long time stability under nonzero mass perturbations and gives rates of decay in time. Apart from the fact that in Theorems 4.1 and 4.3 we are able to do without the structural assumption (H6) of [Z1], we believe that the main interest of the long time stability results here lies in the new methods used to obtain them, which apart from their independent interest also illuminate and unify previous theory. In particular, Theorem 4.2 gives a natural extension to multidimensions of the zero mass approach of $[\mathrm{KK}]$ and, in combination with Theorem 4.5, clarifies the relation of this approach to the one used in [Z1] to treat general perturbations; Theorem 4.4 shows that the approach of $\mathrm{KK}$ can succeed also for general perturbations in sufficiently high dimensions $d \geq 3$. Moreover, in contrast to the Green's function methods of [Z1], the conjugation and degenerate symmetrizer arguments can be readily extended to curved shocks with the aid of pseudodifferential operators. Indeed, in the sequels GMWZ1, GMWZ2, GMWZ3, we apply such arguments to give a rigorous justification of the small viscosity limit for curved multidimensional shocks.

\section{Assumptions}

\subsection{Assumptions on the equations.}

(H1): $f^{j} \in C^{\infty}\left(\mathbb{R}^{n}, \mathbb{R}^{n}\right)$.

(H2): $\sum_{j=1}^{d} d f^{j}\left(U_{R, L}\right) \xi_{j}$ has simple real eigenvalues for $\xi \in \mathbb{R}^{d} \backslash 0$

(strict hyperbolicity of $U_{R, L}$ ). 
Observe that (H2) implies

$$
\text { the eigenvalues } \beta \text { of }-i \sum_{j=1}^{d} d f^{j}\left(U_{R, L}\right) \xi_{j}-|\xi|^{2} \text { satisfy } \Re \beta=-|\xi|^{2} \text {. }
$$

\subsection{Assumption on the shock.}

(H3) $\left(U_{R}, U_{L}, s\right)$ satisfies the Lax shock inequalities La].

Remark 2.1. (1) (H3) implies the eigenvalues of $d f^{1}\left(U_{R, L}\right)$ are nonzero, so $x_{1}=0$ is noncharacteristic. Let $k$ (resp. $l$ ) be the number of positive (resp. negative) eigenvalues of $d f^{1}\left(U_{R}\right)$ (resp. $d f^{1}\left(U_{L}\right)$ ). Then (H3) implies

$$
k+l=n-1 .
$$

(2) The hyperbolicity hypothesis can be weakened, and more general viscosities and types of shocks can be handled by the methods here. In particular, (H2) may be weakened to allow the case of nonstrictly hyperbolic but constant multiplicity systems with stable viscosity matrices treated in [MZ2]; extensions to the variablemultiplicity case are discussed in [MZ2 GMWZ4. Also, (H3) may be weakened to allow overcompressive shocks as in [Z1]; see Remark 10.1] below. (As described in [Z1], undercompressive shocks require a slightly different treatment.) Since most of the difficulties are already present under the above simple assumptions, we will work with these in order to lighten the exposition. We plan to treat more general situations in a future work.

An important consequence of (H3) is:

Proposition 2.1. $\psi$ decays at exponential rate to its endstates. There exist positive constants $C, \beta$ such that

$$
\begin{aligned}
& \left|\psi\left(x_{1}\right)-U_{R}\right| \leq C e^{-\beta x_{1}} \text { for } x_{1}>0, \\
& \left|\psi\left(x_{1}\right)-U_{L}\right| \leq C e^{\beta x_{1}} \text { for } x_{1}<0 .
\end{aligned}
$$

Proof. Since the eigenvalues of $d f^{1}\left(U_{R, L}\right)$ are nonzero, the center manifold of (1.6) at the rest points $U_{R, L}$ is trivial.

2.3. Reduction to a forward problem. Consider the problem

$$
\begin{aligned}
& u_{t}+\sum_{j=1}^{d} f^{j}(u)_{x_{j}}=\Delta u, \\
& \left.u\right|_{t=0}=\psi\left(x_{1}\right)+\epsilon v_{0}(x),
\end{aligned}
$$

and look for $u(x, t)$ of the form

$$
u(x, t)=\psi\left(x_{1}\right)+\epsilon v(x, t) .
$$

Write

$$
f^{j}(\psi+\epsilon v)=f^{j}(\psi)+\epsilon A_{j}\left(x_{1}\right) v+\epsilon^{2} g_{j}(\psi, v, \epsilon), \text { for } j=1, \ldots, d,
$$

where

$$
A_{j}\left(x_{1}\right)=d f^{j}\left(\psi\left(x_{1}\right)\right),
$$

and note that $g_{j}$ is a smooth function of its arguments satisfying

$$
\left|g_{j}(\psi, v, \epsilon)\right| \leq C_{M}|v|^{2} \text { for }|v| \leq M \text {. }
$$


Usually, we will set $A\left(x_{1}\right) \equiv A_{1}\left(x_{1}\right)$. In view of Proposition 2.1] the matrices $A_{j}\left(x_{1}\right)$ decay exponentially to their limiting values at $\pm \infty$.

The problem satisfied by $v(x, t)$ is then

$$
\begin{aligned}
& v_{t}+\left(A\left(x_{1}\right) v\right)_{x_{1}}+\sum_{j=2}^{d} A_{j}\left(x_{1}\right) \partial_{j} v+\epsilon \sum_{j=1}^{d}\left(g_{j}(\psi, v, \epsilon)\right)_{x_{j}}=\Delta v, \\
& \left.v\right|_{t=0}=v_{0} .
\end{aligned}
$$

Next, as in [KK] to obtain a problem with zero initial data we look for $v(x, t)=$ $\tilde{u}+e^{-t} v_{0}(x)$. Drop the tilde on $u$, suppress the harmless $\epsilon$ dependence in $g_{j}$, and write

$$
g_{j}\left(\psi, u+e^{-t} v_{0}\right)=g_{j}\left(\psi, e^{-t} v_{0}\right)+B_{j}(x, t) u+h_{j}(x, t, u)
$$

where

$$
\begin{aligned}
& B_{j}(x, t)=\partial_{u} g_{j}\left(\psi, e^{-t} v_{0}\right), \\
& \left|h_{j}(x, t, u)\right| \leq C_{M}|u|^{2} \text { when }\left|v_{0}, u\right| \leq M .
\end{aligned}
$$

The problem satisfied by $u$ can now be written

$$
\begin{aligned}
& u_{t}+\left(A\left(x_{1}\right) u\right)_{x_{1}}+\sum_{j=2}^{d} A_{j}\left(x_{1}\right) \partial_{j} u+\epsilon \operatorname{div}_{x}(B(x, t) u)+\epsilon \operatorname{div}_{x}(h(x, t, u)) \\
& =\triangle u+e^{-t} v_{0}-\operatorname{div}_{x} \mathcal{A}(x, t)-\epsilon \operatorname{div}_{x}\left(g\left(\psi, e^{-t} v_{0}\right)\right)+\operatorname{div}_{x}\left(e^{-t} \nabla_{x} v_{0}\right), \\
& \left.u\right|_{t=0}=0
\end{aligned}
$$

where

$$
\begin{aligned}
& \mathcal{A}(x, t)=\left(A_{1}\left(x_{1}\right) e^{-t} v_{0}, \ldots, A_{d}\left(x_{1}\right) e^{-t} v_{0}\right), \\
& B(x, t) u=\left(B_{1}(x, t) u, \ldots, B_{d}(x, t) u\right), \text { etc. }
\end{aligned}
$$

Remark 2.2. Note that if $v_{0}=\operatorname{div}_{x} V$ for some $V=\left(V_{1}, \ldots, V_{d}\right)$, the problem (2.12) takes the following form with conservative forcing:

$$
\begin{aligned}
& u_{t}+\left(A\left(x_{1}\right) u\right)_{x_{1}}+\sum_{j=2}^{d} A_{j}\left(x_{1}\right) \partial_{j} u+\epsilon \operatorname{div}_{x}(B(x, t) u)+\epsilon \operatorname{div}_{x}(h(x, t, u)) \\
& =\triangle u+\operatorname{div}_{x} \mathcal{F}(x, t), \\
& \left.u\right|_{t=0}=0,
\end{aligned}
$$

where

$$
\mathcal{F}=e^{-t} V-\mathcal{A}-\epsilon g\left(\psi, e^{-t} v_{0}\right)+e^{-t} \nabla_{x} v_{0} .
$$

In Part 2 we obtain energy estimates for the following linear problem corresponding to (2.12):

$$
\begin{aligned}
& \text { (a) } u_{t}+\left(A\left(x_{1}\right) u\right)_{x_{1}}+\sum_{j=2}^{d} A_{j}\left(x_{1}\right) \partial_{j} u-\triangle u=f, \\
& \text { (b) }\left.u\right|_{t=0}=0
\end{aligned}
$$


2.4. Evans function. We will work mostly in frequency variables so we change notation, replacing $\left(x_{1}, x_{2}, \ldots, x_{d}\right)$ by $\left(x, y_{2}, \ldots, y_{d}\right)$ and letting $(\tau, \eta)$ be dual to $(t, y)$. We will take the Laplace transform in $t$ and set $\lambda=i \tau+\gamma$, where $\gamma \geq 0$.

Extend $u$ and $f$ in (2.16) by zero in $t<0$ and take Fourier-Laplace transforms in $(t, y)$ to get the eigenvalue equation:

$$
\hat{u}_{x x}-(A(x) \hat{u})_{x}-s(x, \lambda, \eta) \hat{u}=\hat{f}(x, \lambda, \eta)
$$

where

$$
s(x, \lambda, \eta)=\sum_{j=2}^{d} A_{j}(x) i \eta_{j}+\lambda I+|\eta|^{2} I .
$$

Next rewrite this as a $2 n \times 2 n$ first order system on $\mathbb{R}$ depending on frequency

$$
\left(\begin{array}{l}
\hat{u} \\
\hat{v}
\end{array}\right)_{x}=\left(\begin{array}{cc}
A(x) & I \\
s(x, \lambda, \eta) & 0
\end{array}\right)\left(\begin{array}{l}
\hat{u} \\
\hat{v}
\end{array}\right)+\left(\begin{array}{c}
0 \\
\hat{f}(x, \lambda, \eta)
\end{array}\right)
$$

or

$$
\mathcal{U}_{x}=\mathcal{G U}+\mathcal{F}
$$

for short.

Notation 2.1. (1) Set $\zeta=(\tau, \gamma, \eta)$. We will sometimes write (with slight abuse) $\zeta=(\lambda, \eta)$ and $f(\lambda, \eta)=f(\zeta)$

(2) Introduce polar coordinates

$$
\zeta=\rho \hat{\zeta}, \text { where } \hat{\zeta}=(\hat{\tau}, \hat{\gamma}, \hat{\eta}) \text { and } \hat{\zeta} \in S^{d} .
$$

We will always take $\gamma \geq 0$, so define $S_{+}^{d}=S^{d} \cap\{\hat{\gamma} \geq 0\}$.

Remark 2.3. Observe that smooth functions $f(\zeta)$ of $\zeta \in \mathbb{R}^{d+1}$ can be rewritten as smooth functions $f(\hat{\zeta}, \rho)$ with $(\hat{\zeta}, \rho) \in S^{d} \times \overline{\mathbb{R}}_{+}$. However, when $f(\hat{\zeta}, 0)$ is not constant on $S^{d}$, the function $f(\zeta)$ corresponding to $f(\hat{\zeta}, \rho)$ is not continuous at $\zeta=0$.

In order to define the Evans function we recall the following lemma from [ZS]:

Lemma 2.1. For $\eta \in \mathbb{R}^{d-1}, \Re \lambda>0$, there exist bases of solutions

$$
\left\{\mathcal{U}_{1}^{R}, \cdots, \mathcal{U}_{n}^{R}\right\},\left\{\mathcal{U}_{1}^{L}, \cdots, \mathcal{U}_{n}^{L}\right\}
$$

of (2.19) with $\mathcal{F}=0$, spanning the stable/unstable manifolds at $x=+\infty /-\infty$, respectively, such that

$$
\left.\mathcal{D}(\lambda, \eta) \equiv \operatorname{det}\left(\mathcal{U}_{1}^{R}, \cdots, \mathcal{U}_{n}^{R}, \mathcal{U}_{1}^{L}, \cdots, \mathcal{U}_{n}^{L}\right)\right|_{x=0}
$$

is analytic in $(\lambda, \eta)$ and continuously extendible to $\Re \lambda=0$.

Definition 2.1. $\mathcal{D}$ is called the Evans-Lopatinski determinant (or Evans function for short) for the problem (2.19). Here and henceforth we always normalize the columns appearing in (2.22) so that they of are of size $\sim 1$ for $\rho$ near 0 .

Remark 2.4. (1) Note that nonvanishing of $\mathcal{D}$ in $\Re \lambda>0$ is necessary even for linearized stability. Linear dependence implies existence of a solution $\mathcal{U}=(\hat{u}, \hat{v})$ to the homogeneous problem decaying at both $\pm \infty$, and thus of an exponentially unstable solution $\hat{w}(x, t, \eta)=e^{\lambda t} \hat{u}(x, \lambda, \eta)$ of [2.16) (a) with $f=0$.

(2) In $\rho>0$ we may write $D(\zeta)=D(\hat{\zeta}, \rho)$. In Lemma 5.1 in [ZS] it is shown that $D(\hat{\zeta}, \rho)$ is analytic in $\{\hat{\gamma}>0, \rho>0\}$. In view of Remark 7.1 below, $\mathcal{D}$ and $\mathcal{D}_{\rho}$ 
are continuously extendible to $\{\hat{\gamma} \geq 0, \rho \geq 0\}$. In addition, Proposition 5.3 of [ZS] implies

$$
D(\hat{\zeta}, \rho)=C \beta \Delta(\hat{\zeta}) \rho+o(\rho)
$$

as $\rho \rightarrow 0$, for some $C \neq 0$. Here $\beta$ is nonvanishing if and only if the stable/unstable manifolds for $U_{R} / U_{L}$ of the travelling wave ODE (1.6) are transverse at the connection $\psi$, while $\Delta(\hat{\zeta})$ is the Lopatinski-Kreiss-Majda determinant for the ideal shock problem linearized at $\left(U_{R}, U_{L}, s\right)$.

The computation giving (2.23) shows that

$$
D(\hat{\zeta}, \rho) \in C\left(S_{+}^{d}, C^{1}\left(\overline{\mathbb{R}}_{+}\right)\right) .
$$

(3) The vanishing of $D(\hat{\zeta}, 0)$ reflects the fact that at $\rho=0$ the homogeneous version of (2.19) has the solution $\mathcal{U}=(\phi, 0)$, where $\phi=\psi^{\prime}$ (differentiate (1.6) twice). This solution is fast decaying at both $\pm \infty$. It will be convenient later to normalize

$$
\mathcal{U}_{1}^{R}(x, \hat{\zeta}, 0)=\mathcal{U}_{n}^{L}(x, \hat{\zeta}, 0)=(\phi(x), 0)
$$

(4) Lemma 2.1 follows from the Gap Lemma of [GZ and Proposition 5.1 below.

\subsection{Assumption on the viscous profile.}

(H4) $D(\hat{\zeta}, \rho)$ vanishes to precisely first order at $\rho=0$ (where it must vanish) for all $\hat{\zeta} \in S_{+}^{d}$ and has no other zeros in $S_{+}^{d} \times \overline{\mathbb{R}}_{+}$.

Remark 2.5. In view of the above remarks $D(\hat{\zeta}, \rho)$ vanishes to precisely first order at $\rho=0$ if and only if both $\beta \neq 0$ and $\Delta(\hat{\zeta}) \neq 0$ on $S_{+}^{d}$, i.e., the viscous profile is a transversal connection of the travelling-wave ODE and the ideal shock $\left(U_{R}, U_{L}, s\right)$ satisfies Majda's uniform Lopatinski condition.

\subsection{Admissible perturbations.}

Notation 2.2. Consider $v(x, y)$ defined for $(x, y) \in \mathbb{R}^{d}$.

(1) $\langle v\rangle_{2 ; p}^{2}=\sum_{|\alpha| \leq p}\left|\partial_{(x, y)}^{\alpha} v(x, y)\right|_{L^{2}(x, y)}^{2}$.

(2) $\langle v\rangle_{(2,1) ; p}^{2}=\sum_{|\alpha| \leq p}\left|\partial_{(x, y)}^{\alpha} v(x, y)\right|_{L^{2}\left(x, L^{1}(y)\right)}^{2}$.

(3) $\langle v\rangle_{1 ; p}=\sum_{|\alpha| \leq p}\left|\partial_{(x, y)}^{\alpha} v(x, y)\right|_{L^{1}(x, y)}$.

Remark 2.6. We caution the reader not to confuse the above notation with the commonly used $W^{k, p}$ notation for Sobolev spaces, where $k$ is an order of differentiation and $p$ is an $L^{p}$ exponent.

In each of our three main theorems we will use perturbations from one of the following admissible classes. 
Definition 2.2. For $p \in\{0,1,2, \ldots\}$ define the sets of perturbations $\mathcal{A}_{p}^{I}, \mathcal{A}_{p}^{I I}$, $\mathcal{A}_{p}^{I I I}, \mathcal{A}_{p}^{I V}, \mathcal{A}_{p}^{V}$ by

$$
\begin{aligned}
& \mathcal{A}_{p}^{I}=\left\{v_{0}(x, y)=\operatorname{div}_{(x, y)} V, \text { where } V=\left(V_{1}, \ldots, V_{d}\right),\right. \\
& \text { satisfies } \left.\langle V\rangle_{2 ; p+2}+\langle V\rangle_{(2,1) ; 2}<\infty\right\} \text {, } \\
& \mathcal{A}_{p}^{I I}=\left\{v_{0}(x, y):\left\langle v_{0}\right\rangle_{2 ; p+2}+\left\langle v_{0}\right\rangle_{(2,1) ; 2}<\infty\right\}, \\
& \mathcal{A}_{p}^{I I I}=\left\{v_{0}(x, y):\left\langle v_{0}\right\rangle_{2 ; p+2}+\left\langle v_{0}\right\rangle_{1 ; 2}<\infty\right\} \text {, } \\
& \mathcal{A}_{p}^{I V}=\left\{v_{0}(x, y)=\operatorname{div}_{(x, y)} V \text {, where } V=\left(V_{1}, \ldots, V_{d}\right)\right. \\
& \text { satisfies } \left.\langle V\rangle_{2 ; p+2}+\langle V\rangle_{1 ; 2}<\infty\right\} \text {, } \\
& \mathcal{A}^{V}=\left\{v_{0}(x, y):\left\langle v_{0}\right\rangle_{\infty ; 0}+\left\langle v_{0}\right\rangle_{1 ; 0}<\infty\right\} .
\end{aligned}
$$

2.7. Assumption on the perturbation $v_{0}$. Each theorem will make one of the following assumptions:

$$
\begin{aligned}
& (\mathrm{H} 5)^{I} \quad v_{0} \in \mathcal{A}_{p}^{I} \text { for some } p>\frac{d}{2} . \\
& (\mathrm{H} 5)^{I I} \quad v_{0} \in \mathcal{A}_{p}^{I I} \text { for some } p>\frac{d}{2} . \\
& (\mathrm{H} 5)^{I I I} \quad v_{0} \in \mathcal{A}_{p}^{I I I} \text { for some } p>\frac{d}{2} . \\
& (\mathrm{H} 5)^{I V} \quad v_{0} \in \mathcal{A}_{p}^{I V} \text { for some } p>\frac{d}{2} . \\
& (\mathrm{H} 5)^{V} \quad v_{0} \in \mathcal{A}^{V} .
\end{aligned}
$$

\section{Auxiliary assumption on the ShOCK}

In parts of our treatment of nonzero mass perturbations, we shall have to augment the above assumptions with an additional structural assumption (H6) as in [Z1] (also called $(\mathrm{H} 6)$ there). Let $(\xi, \tau, \eta)$ denote (real) variables dual to $(x, t, y)$.

Notation 3.1. Let $A( \pm \infty)=\lim _{x \rightarrow \pm \infty} A(x)$ and define $A_{j}( \pm \infty), j=2, \ldots, d$, similarly. Let the matrix symbols of the linearized invisicid limiting operators be

$$
L_{ \pm}(\xi, \tau, \eta)=i \tau I+A( \pm \infty) i \xi+\sum_{j=2}^{d} A_{j}( \pm \infty) i \eta_{j}
$$

and the corresponding scalar symbols be

$$
p_{ \pm}(\xi, \tau, \eta)=\operatorname{det} L_{ \pm}(\xi, \tau, \eta) .
$$

Definition 3.1. Define the glancing set $\mathcal{G}$ to be the set of $(\tau, \eta) \in \mathbb{R}^{d} \backslash 0$ such that for at least one choice of sign the equation $p_{ \pm}(\xi, \tau, \eta)=0$ has a real root $\xi$ of multiplicity $\geq 2$.

Clearly, at any point $\left(\tau_{0}, \eta_{0}\right) \in \mathcal{G}$ at least one real root $\xi$ of $p_{ \pm}(\xi, \tau, \eta)=0$ has a branch singularity. (The degree of singularity with respect to $\tau$ ( $\eta$ held fixed) is equal to the integer $s$ in (3.5) below.)

The hyperbolicity assumption (H2) implies there exist real functions $\tau_{1}^{ \pm}(\xi, \eta), \ldots$, $\tau_{n}^{ \pm}(\xi, \eta)$, smooth and homogeneous of degree one in $(\xi, \eta) \neq 0$, such that

$$
\begin{aligned}
& \tau_{1}^{ \pm}<\cdots<\tau_{n}^{ \pm} \text {and } \\
& p_{ \pm}(\tau, \xi, \eta)=i^{n}\left(\tau-\tau_{1}^{ \pm}(\xi, \eta)\right) \cdots\left(\tau-\tau_{n}^{ \pm}(\xi, \eta)\right) .
\end{aligned}
$$


If $\left(\tau_{0}, \eta_{0}\right) \in \mathcal{G}$, there exist $\xi_{0}$ and for at least one choice of sign a $\tau_{j}^{ \pm}$(with $j$ uniquely determined by the choice of \pm and $\left.\left(\xi_{0}, \tau_{0}, \eta_{0}\right)\right)$ such that (dropping \pm )

$$
\begin{aligned}
& \tau_{0}=\tau_{j}\left(\xi_{0}, \eta_{0}\right) \text { and } \\
& \partial_{\xi} \tau_{j}\left(\xi_{0}, \eta_{0}\right)=0 .
\end{aligned}
$$

Moreover, the multiplicity of $\xi_{0}$ as a root of $p\left(\xi_{0}, \tau_{0}, \eta_{0}\right)=0$, and thus the degree of singularity (with respect to $\tau$ ) of the associated branch point, is equal to $s$ $(2 \leq s \leq n)$ if and only if

$$
\begin{aligned}
& \partial_{\xi}^{k} \tau_{j}\left(\xi_{0}, \eta_{0}\right)=0, \text { for } k=1, \ldots, s-1, \text { but } \\
& \partial_{\xi}^{s} \tau_{j}\left(\xi_{0}, \eta_{0}\right) \neq 0 .
\end{aligned}
$$

Note that this implies at the same time that $\partial_{\xi} \tau_{j}\left(\cdot, \eta_{0}\right)$ has no roots nearby $\xi_{0}$ other than $\xi_{0}$ itself.

Clearly, (3.5) and the implicit function theorem imply that for any such $\left(\tau_{0}, \xi_{0}, \eta_{0}\right)$ and function $\tau_{j}$, there exists a function $\xi(\eta)$ such that locally near $\left(\xi_{0}, \eta_{0}\right)$

$$
\partial_{\xi}^{s-1} \tau_{j}(\xi, \eta)=0 \text { precisely when } \xi=\xi(\eta) .
$$

Note that $\xi(\eta)$ is smooth and homogeneous of degree one away from $\eta=0$. We can now state the auxiliary assumption (H6):

(H6) For any $\left(\tau_{0}, \eta_{0}\right) \in \mathcal{G}$, corresponding root $\xi_{0}$ of multiplicity $s$, and functions $\tau_{j}$ and $\xi(\eta)$ as above, we have

$$
\partial_{\xi}^{k} \tau_{j}(\xi(\eta), \eta)=0 \text { for } k=1, \ldots, s-1 \text { and } \eta \text { near } \eta_{0} .
$$

In other words $\xi_{0}$ persists as a root $\xi(\eta)$ of multiplicity $s$ of

$$
p\left(\xi(\eta), \tau_{j}(\xi(\eta), \eta), \eta\right)=0
$$

for $\eta$ near $\eta_{0}$, and (by the remark below (3.5)) there are no other nearby roots of multiplicity $>1$.

A compactness argument using the fact that $\mathcal{G}$ is a closed conic set shows that under the assumption (H6) all such branch singularities are confined to a finite union of surfaces

$$
\tau=\tau_{j, l}(\eta) \equiv \tau_{j}\left(\xi_{l}(\eta), \eta\right)
$$

on which the singularity (with respect to $\tau$ ) has order equal to $s_{l}$, the multiplicity of the root $\xi_{l}(\eta)$; this is the version of (H6) stated in [Z1]. We will usually relabel and replace the double index $j, l$ by a single index as in $\tau=\tau_{k}(\eta)$. Note that graphs $\tau_{k}$ may well intersect.

Remark 3.1. (1) The statements of this subsection require only slight modification when the assumption of strict hyperbolicity (H2) is relaxed to the following more general hypothesis of [Z1], [MZ1]:

$\left(\mathrm{H} 2^{\prime}\right): \sum_{j=1}^{d} d f^{j}\left(U_{R, L}\right) \xi_{j}$ has semisimple real eigenvalues of constant multiplicity for $\xi \in \mathbb{R}^{d} \backslash 0$ (nonstrict hyperbolicity with constant multiplicity).

In this case the multiplicity of $\xi_{0}$ as a root of $p\left(\xi_{0}, \tau_{0}, \eta_{0}\right)=0$ is some integer multiple of $s$ as in (3.5)).

(2) Condition (H6) is automatic in the cases $d=1,2$ and also in any dimension for rotationally invariant problems. In 1D the glancing set is empty. In the $2 \mathrm{D}$ case the homogeneity of $\tau_{j}$ and its derivatives implies that the ray through $\left(\xi_{0}, \eta_{0}\right)$ is the graph of $\xi(\eta)$ and that (H6) holds there. (H6) also clearly holds if no real root 
$\xi$ of $p(\xi, \tau, \eta)=0$ has multiplicity $>2$, in particular in the case that all eigenvalues $\tau_{j}(\xi, \eta)$ are linear or convex/concave in their dependence on $\xi$.

(3) In the equations of gas- and magnetohydrodynamics (MHD), all characteristics are linear combinations of $(\xi, \eta)$ and $|\xi, \eta|$, hence the above results show that (H6) is valid whenever the constant multiplicity assumption (H2') applies. Thus, we see that (H6), though mathematically restrictive, nonetheless allows important physical applications.

(4) The word glancing is used in Definition 3.1 since null bicharacteristics of $p$ through points $(\xi, \tau, \eta)$ with $\xi$ a root of multiplicity $\geq 2$ run parallel to $x=0$.

(5) In [Z1], there were made besides (H6) two additional auxiliary assumptions (H5) (different from our (H5) here) and (H7); however, these hold automatically in the case of uniform stability considered here (uniform inviscid stability, in the language of the reference). Thus, within the context under consideration, we make here exactly the same assumptions as were made in [Z1], and we will obtain the same results, though by quite different techniques.

\section{Main Results}

Recall the definition of nonlinear stability of the viscous profile $\psi$ with respect to a family of perturbations (Definition 1.1).

Theorem 4.1 (Zero mass, $d \geq 3$ ). Assume (H1), (H2), (H3), (H4), and (H5) ${ }^{I}$, where the number of space dimensions is $d \geq 3$. Then the viscous profile $\psi$ is nonlinearly stable with respect to $\mathcal{A}_{p}^{I}$.

Theorem 4.2 (Zero mass, $d \geq 1$ ). Assume (H1), (H2), (H3), (H4), (H5) ${ }^{I V}$, and (H6), where the number of space dimensions is $d \geq 1$. Then the viscous profile $\psi$ is nonlinearly stable with respect to $\mathcal{A}_{p}^{I V}$.

Theorem 4.3 (Nonzero mass, $d \geq 5$ ). Assume (H1), (H2), (H3), (H4), and $(H 5)^{I I}$, where the number of space dimensions is $d \geq 5$. Then the viscous profile $\psi$ is nonlinearly stable with respect to $\mathcal{A}_{p}^{I I}$.

Theorem 4.4 (Nonzero mass, $d \geq 3$ ). Assume (H1), (H2), (H3), (H4), (H5) ${ }^{I I I}$, and (H6), where the number of space dimensions is $d \geq 3$. Then the viscous profile $\psi$ is nonlinearly stable with respect to $\mathcal{A}_{p}^{I I I}$.

Theorem 4.5 (Nonzero mass, $d \geq 2$ ). Assume (H1), (H2), (H3), (H4), (H5) ${ }^{V}$, and (H6), where the number of space dimensions is $d \geq 2$. Then the viscous profile $\psi$ is nonlinearly stable with respect to $\mathcal{A}^{V}$. Moreover, the perturbation $u$ decays in $L^{p}, p \geq 2$, at the rate $|u|_{p}(t) \leq C(p, d)(1+t)^{-\frac{d-1}{2}\left(1-\frac{1}{p}\right)}$ of a $(d-1)$-dimensional heat kernel, where $C(p, d)$ is monotone increasing in $p$, finite for $p<\infty$, and uniformly bounded for $d \geq 3$.

Remark 4.1. (1) Clearly, Theorem 4.5 implies Theorem 4.4; however, we shall prove them by rather different arguments, and so we maintain the distinction for easy referencing.

(2) The nonzero mass case for $d=1$ is treated by estimation of Green's functions in $[\mathrm{ZH}, \mathrm{Z2}$.

(3) We can drop (H6) in the above theorems when $d=1$ or 2 (Remark 3.1(2)).

(4) (H1) can be weakened to $f^{j} \in C^{\frac{[d]}{2}+5}\left(\mathbb{R}^{n}, \mathbb{R}^{n}\right)$ for Theorems 4.144.4 and $f^{j} \in C^{2}$ for Theorem 4.5 


\section{Part 2. Algebraic preparation}

\section{Doubling And COnJugation}

As explained in the introduction, we want to rewrite the $2 n \times 2 n$ system 2.19 ) on $\mathbb{R}$ as an equivalent $4 n \times 4 n$ "doubled" boundary problem on $x \geq 0$. We have (with $(x, y)$ in place of $\left(x_{1}, x^{\prime}\right)$ )

$$
\begin{aligned}
& U_{x}-G(x, \lambda, \eta) U=F, \\
& \Gamma U=0 \text { on } x=0,
\end{aligned}
$$

where in the \pm notation (1.1),

$$
\begin{aligned}
& U(x, \lambda, \eta)=\left(\mathcal{U}_{+}, \mathcal{U}_{-}\right), \\
& G(x, \lambda, \eta)=\left(\begin{array}{cc}
\mathcal{G}_{+} & 0 \\
0 & -\mathcal{G}_{-}
\end{array}\right), \\
& F=\left(\begin{array}{c}
\mathcal{F}_{+} \\
-\mathcal{F}_{-}
\end{array}\right), \text {and } \\
& \Gamma U=\mathcal{U}_{+}-\mathcal{U}_{-} .
\end{aligned}
$$

Recall, for $x \geq 0$

$$
\mathcal{G}_{ \pm}(x, \lambda, \eta)=\left(\begin{array}{cc}
A( \pm x) & I \\
s( \pm x, \lambda, \eta) & 0
\end{array}\right)
$$

Henceforth, we will drop the script notation and write $U=\left(U_{+}, U_{-}\right)$.

Remark 5.1. Both here and in the sequel GMWZ1 there are several advantages to working with the doubled boundary problem. Instead of having two distinct limiting problems (as $x \rightarrow \pm \infty$ ), after doubling we have just one (5.13). This will allow us to conjugate the original problem on the whole line to a single constant coefficient problem on $x \geq 0$. The doubled boundary formulation allows one readily to construct high order approximate solutions for the small viscosity problem with prepared data $(\mathrm{GW}])$. Perhaps most important, we are now in a position to use the machinery of Kreiss-type symmetrizers to prove energy estimates.

The limiting constant coefficient problem plays an essential role in our analysis, and we must first understand the spectral properties of the limiting matrix:

$$
G(\infty, \lambda, \eta)=\left(\begin{array}{cc}
\mathcal{G}_{+}(\infty, \lambda, \eta) & 0 \\
0 & -\mathcal{G}_{-}(\infty, \lambda, \eta)
\end{array}\right)
$$

where

$$
\mathcal{G}_{ \pm}(\infty, \lambda, \eta)=\lim _{x \rightarrow+\infty} \mathcal{G}_{ \pm}(x, \lambda, \eta)=\left(\begin{array}{cc}
A( \pm \infty) & I \\
s( \pm \infty, \lambda, \eta) & 0
\end{array}\right) .
$$

Proposition 5.1 (Spectral properties of $\mathcal{G}(\infty, \lambda, \eta)$, [Z1], [ZS]).

(1) When $\rho>0$ and $\gamma \geq 0, G(\infty, \lambda, \eta)$ has $2 n$ eigenvalues counted with multiplicities in $\Re \mu>0$ and $2 n$ eigenvalues in $\Re \mu<0$.

(2) $G(\infty, 0,0)$ has 0 as a semisimple eigenvalue of multiplicity $2 n$. The nonvanishing eigenvalues (fast modes) are those of $A(+\infty)$ ( $k$ positive, $n-k$ negative) and $-A(-\infty)$ (l positive, $n-l$ negative).

(3) Consider the multiple zero eigenvalue of $G(\infty, \hat{\zeta}, 0)$ (polar coordinates). For $\hat{\gamma}>\delta>0$, this eigenvalue splits for $\rho>0$ small into $k+l=n-1$ slow decaying 
modes

$$
\mu=c_{\delta} \rho+O\left(\rho^{2}\right) \text { where } \Re c_{\delta}<0
$$

and $(n-k)+(n-l)=n+1$ slow growing modes $\left(\Re c_{\delta}>0\right)$.

Here "decaying" and "growing" refer to the corresponding exponential solutions $e^{\mu x} v$.

Proof. (1) We focus on $\mathcal{G}_{+}(\infty, \lambda, \eta)$; a parallel argument handles $-\mathcal{G}_{-}(\infty, \lambda, \eta)$.

Note that $\mu$ is an eigenvalue of $\mathcal{G}_{+}(\infty, \lambda, \eta)$ if and only if

$$
\left[\mu^{2}-|\eta|^{2}-\mu A(+\infty)-i \sum_{2}^{d} A_{j}(+\infty) \eta_{j}-\lambda\right] v=0
$$

for some nonzero $v$. Setting $\mu=i \eta_{1}, \eta_{1} \in \mathbb{R}$ yields

$$
\operatorname{det}\left[-\left|\eta_{1}, \eta\right|^{2}-i \sum_{1}^{d} A_{j}(+\infty) \eta_{j}-\lambda\right]=0
$$

which by (2.1) has no solution with $\Re \lambda \geq 0$, except for $\left(\eta_{1}, \eta\right)=0, \lambda=0$. Thus, there are no eigenvalues with $\Re \mu=0$ when $\rho>0, \gamma \geq 0$, and the number of eigenvalues in each of $\Re \mu>0$ and $\Re \mu<0$ is constant then. We may choose $\tau=0, \eta=0$ and $\gamma$ large to obtain an obvious count.

(2) This is clear since $\mathcal{G}_{+}(\infty, 0,0)=\left(\begin{array}{cc}A(+\infty) & I \\ 0 & 0\end{array}\right)$.

(3) Consider the characteristic equation in polar coordinates (drop the hats)

$$
\left[\mu^{2}-\rho^{2}|\eta|^{2}-\mu A(+\infty)-i \rho \sum_{2}^{d} A_{j}(+\infty) \eta_{j}-\rho \lambda\right] v=0
$$

and posit the expansions

$$
\mu=c \rho+O\left(\rho^{2}\right), \quad v=r+O(\rho) .
$$

Compare terms of order $\rho$ to obtain

$$
\begin{aligned}
& \left(c A(+\infty)+i \sum_{2}^{d} A_{j}(+\infty) \eta_{j}+\lambda\right) r=0, \text { or } \\
& {\left[c+\left(i \sum_{2}^{d} A_{j}(+\infty) \eta_{j}+\lambda\right) A(+\infty)^{-1}\right] A(+\infty) r=0 .}
\end{aligned}
$$

Thus, $c$ is an eigenvalue of $-\left(i \sum_{2}^{d} A_{j}(+\infty) \eta_{j}+\lambda\right) A(+\infty)^{-1}$, which by hyperbolicity has no center manifold for $\gamma>0$. So the stable/unstable roots $\Re c<0 / \Re c>0$ separate to first order in $\rho$. They may be counted by setting $\eta=0$ and using the fact that $A(+\infty)$ has $k$ positive eigenvalues.

The conjugation argument is based on the following lemma [MZ1]:

Lemma 5.1. Let $\Omega=\{(\lambda, \eta):|\lambda, \eta| \leq C, \gamma \geq 0\}$. There is a matrix $W(x, \lambda, \eta)$ defined and smooth on $[0, \infty) \times \Omega$ such that

(a) $W^{-1}$ is uniformly bounded and there is a $\theta>0$ such that

$$
W(x, \lambda, \eta)=I+O\left(e^{-\theta x}\right) .
$$


(b) $W$ satisfies

$$
\partial_{x} W=G(x) W(x)-W(x) G(\infty) .
$$

The proof involves an application of the Gap Lemma [GZ to the operator $\operatorname{ad} G(\infty)=[G(\infty), \cdot]$.

The substitution $U=W V$ transforms the equation (5.1) into

$$
\begin{aligned}
& V_{x}-G(\infty, \lambda, \eta) V=W^{-1} F, \\
& \tilde{\Gamma}(x, \lambda, \eta) V=0 \text { on } x=0,
\end{aligned}
$$

where $\tilde{\Gamma}(x, \lambda, \eta) V=\Gamma W(x, \lambda, \eta) V$. Thus, estimates for (5.13) imply estimates for (5.1).

We will refer to $W$ as the $M Z$ conjugator.

\section{BlOCK STRUCTURE AND STANDARD SYMMETRIZERS}

6.1. Block structure. In this subsection we present some results for the viscous shock problem whose proofs are essentially identical to results in [MZ1 for the viscous Dirichlet problem. We will recall or sketch some proofs and otherwise refer the reader to MZ1. $G(\infty)$ is the limiting $4 n \times 4 n$ constant coefficient matrix defined in (5.4). Recall $\zeta=(\tau, \gamma, \eta)$.

Lemma 6.1. There is a $C^{\infty}$ invertible matrix $T(\zeta)$ defined on a neighborhood of $\zeta=0$ such that $T^{-1} G(\infty) T$ has the block diagonal form

$$
T^{-1} G(\infty) T=\left(\begin{array}{cccc}
P_{R} & 0 & 0 & 0 \\
0 & H_{R} & 0 & 0 \\
0 & 0 & P_{L} & 0 \\
0 & 0 & 0 & H_{L}
\end{array}\right) \equiv G_{1}(\infty),
$$

where $H_{R}, H_{L}, P_{R}$, and $P_{L}$ are $C^{\infty}$ functions of $\zeta$ satisfying

$$
\begin{aligned}
& H_{R}(0)=0, H_{L}(0)=0, P_{R}(0)=A(+\infty), P_{L}(0)=-A(-\infty), \\
& H_{R}(\zeta)=-s(+\infty, \zeta) A(+\infty)^{-1}+O\left(|\zeta|^{2}\right), \\
& H_{L}(\zeta)=s(-\infty, \zeta) A(-\infty)^{-1}+O\left(|\zeta|^{2}\right),
\end{aligned}
$$

and

$$
T(0)=\left(\begin{array}{cccc}
I & -A(+\infty)^{-1} & 0 & 0 \\
0 & I & 0 & 0 \\
0 & 0 & I & A(-\infty)^{-1} \\
0 & 0 & 0 & I
\end{array}\right)
$$

The eigenvalues of $P_{R}(\zeta)$ and $P_{L}(\zeta)$ satisfy $|\Re \mu|>C>0$ on some neighborhood of $\zeta=0$.

Proof. We give the argument for the $\mathcal{G}_{+}(\infty, \zeta)$ block in (5.4), the other block being treated similarly.

Proposition 5.1 (including its proof) shows that for small $|\zeta|$, the eigenvalues of $\mathcal{G}_{+}(\infty, \zeta)$ may be grouped into $n$ fast modes and $n$ slow modes (fast and slow having been defined in that proposition). The nonvanishing (i.e., fast) eigenvalues 
are those of $A(+\infty)$, so there is a smooth family of matrices $T_{R}(\zeta)$ defined for $\zeta$ small such that

$$
T_{R}^{-1} \mathcal{G}_{+}(\infty, \zeta) T_{R}=\left(\begin{array}{cc}
P_{R} & 0 \\
0 & H_{R}
\end{array}\right)
$$

where $H_{R}(0)=0, P_{R}(0)=A(+\infty)$. Here $T_{R}$ is of course not uniquely determined, but it may be chosen such that

$$
T_{R}(0)=\left(\begin{array}{cc}
I & -A(+\infty)^{-1} \\
0 & I
\end{array}\right) .
$$

This together with a direct perturbation computation shows that the eigenvalues of $\mathcal{G}_{+}(\infty, \zeta)$ close to 0 correspond to a matrix of the form $H_{R}(\zeta)$ given in (6.2).

The eigenvalues of $P_{R}(\zeta)$ have the stated property since the eigenvalues of $A(+\infty)$ are nonvanishing.

Remark 6.1. (1) Observe that the matrix $-s(+\infty, \zeta) A(+\infty)^{-1}$ appeared already in the last paragraph of the proof of Proposition 5.1.

(2) Our expressions for $T(0)$ and $H_{R}(\zeta)$ differ slightly from the corresponding expressions in Lemma 2.7 of [MZ1]. For example, $A(+\infty)^{-1}$ occurs to the right of $-s(+\infty, \zeta)$ in (6.2) instead of to the left as in MZ1. This is because our reduction to a first order system leads to $\mathcal{G}(x, \lambda, \eta)$ as in (2.18), while the corresponding matrix in [MZ1] (in our notation) is

$$
\left(\begin{array}{ll}
0 & I \\
s & A
\end{array}\right)
$$

In the following proposition we use the polar coordinate notation introduced in (2.20). Set

$$
H_{R}(\zeta)=\rho \hat{H}_{R}(\hat{\zeta}, \rho)
$$

and do similiarly for $H_{L}(\zeta)$. Conjugation by a constant coefficient matrix $T_{1}$ (with only zeros and ones) changes $G_{1}$ in (6.1) to $T_{1}^{-1} G_{1} T_{1}=$

$$
G_{2}(\infty, \zeta)=\left(\begin{array}{cccc}
P_{R} & 0 & 0 & 0 \\
0 & P_{L} & 0 & 0 \\
0 & 0 & H_{R} & 0 \\
0 & 0 & 0 & H_{L}
\end{array}\right) .
$$

Proposition 6.1 (Block structure). For all $\underline{\hat{\zeta}}$ with $\underline{\hat{\gamma}} \geq 0$ there is a neighborhood $\omega$ of $(\underline{\hat{\zeta}}, 0)$ in $S^{d} \times \overline{\mathbb{R}}_{+}$and there are $C^{\infty}$ matrices $T_{2}(\overline{\hat{\zeta}}, \rho)$ on $\omega$ such that $T_{2}^{-1} G_{2} T_{2}$ has the block diagonal structure

$$
T_{2}^{-1} G_{2} T_{2}=\left[\begin{array}{ccc}
P_{+}(\zeta) & 0 & 0 \\
0 & P_{-}(\zeta) & 0 \\
0 & 0 & H_{B}(\hat{\zeta}, \rho)
\end{array}\right] \equiv G_{B}(\infty) .
$$

Here the eigenvalues of $P_{+}$(resp. $P_{-}$) belong to a compact set in $\Re \mu>0$ (resp. $\Re \mu<0$ ) and in addition

$$
\Re P_{+}=\frac{1}{2}\left(P_{+}+P_{+}^{*}\right) \geq c I \text { and }-\Re P_{-} \geq c I \text { on } \omega
$$

for some $c>0$. 
We have $H_{B}(\hat{\zeta}, \rho)=\rho \hat{H}_{B}(\hat{\zeta}, \rho)$ with

$$
\hat{H}_{B}(\hat{\zeta}, \rho)=\left[\begin{array}{ccc}
Q_{1} & \cdots & 0 \\
\vdots & \ddots & \vdots \\
0 & \cdots & Q_{p}
\end{array}\right](\hat{\zeta}, \rho) .
$$

The blocks $Q_{k}$ are $\nu_{k} \times \nu_{k}$ matrices which satisfy one of the following conditions:

(i) $\Re Q_{k}$ is positive definite.

(ii) $\Re Q_{k}$ is negative definite.

(iii) $\nu_{k}=1, \Re Q_{k}=0$ when $\hat{\gamma}=\rho=0$, and $\partial_{\hat{\gamma}}\left(\Re Q_{k}\right) \partial_{\rho}\left(\Re Q_{k}\right)>0$.

(iv) $\nu_{k}>1, Q_{k}$ has purely imaginary coefficients when $\hat{\gamma}=\rho=0$, there is $\mu_{k} \in \mathbb{R}$ such that

$$
Q_{k}(\hat{\zeta}, 0)=i\left[\begin{array}{cccc}
\mu_{k} & 1 & 0 & \\
0 & \mu_{k} & \ddots & 0 \\
& \ddots & \ddots & 1 \\
& & \cdots & \mu_{k}
\end{array}\right],
$$

and the lower left corner a of $Q_{k}$ satisfies $\partial_{\hat{\gamma}}(\Re a) \partial_{\rho}(\Re a)>0$.

Moreover, the matrix $T_{2}$ can be taken of the form

$$
T_{2}(\hat{\zeta}, \rho)=\left(\begin{array}{cc}
T_{P}(\zeta) & 0 \\
0 & T_{H}(\hat{\zeta}, \rho)
\end{array}\right),
$$

for $C^{\infty}$ functions $T_{P}$ and $T_{H}$ (recall Remark 2.3).

Sketch of proof. The ability to choose $T_{P}(\zeta)$ conjugating the $\left(P_{R}, P_{L}\right)$ block in $G_{2}$ to the $\left(P_{+}, P_{-}\right)$block in $G_{B}$ follows directly from the nonvanishing of the eigenvalues of $P_{R}, P_{L}$ as described in Proposition 6.1.

The blocks $H_{R}$ and $H_{L}$ are conjugated separately to block structure as in [MZ1]. Thus, there is a $k_{0}$ such that the blocks $Q_{1}, \ldots, Q_{k_{0}}$ in $\hat{H}_{B}$ correspond to $H_{R}$, while blocks $Q_{k_{0}+1}, \ldots, Q_{p}$ correspond to $H_{L}$.

The argument in [MZ1] is a modification of the classic perturbation argument of Kreiss $[\mathbf{K}]$, the difference being that now the perturbation is performed with respect to the parameters $\hat{\gamma}$ and $\rho$, instead of just $\hat{\gamma}$ as in [K]. A key point, here as in [MZ1, is that the assumptions on the original parabolic system, in particular (2.1), imply that the derivatives appearing in (iii) and (iv) above are nonzero and of the same sign. In [K] there was one derivative to consider, $\partial_{\hat{\gamma}} \Re Q_{k}$ (resp. $\partial_{\hat{\gamma}} \Re a$ ), and this was nonzero as a consequence of his strict hyperbolicity assumption. The sign condition in (iii) and (iv) allows one to construct symmetrizers by a small modification of the ansatz used in $[\mathrm{K}]$. (An extra term is added to the $k$ th block of the symmetrizer corresponding to the extra $\rho$ parameter.)

Definition 6.1. Blocks satisfying condition (iv) in the above theorem will be referred to as glancing blocks. These correspond to coalescing eigenvalues.

6.2. Decompositions of $\mathbb{C}^{4 n}$. The conjugation of $G(\infty, \zeta)$ to block structure induces decompositions of $\mathbb{C}^{4 n}$ that are important in the construction of the symmetrizer.

Definition 6.2. For $\zeta \neq 0$ let $\mathbb{F}_{ \pm}(\zeta)$ denote the direct sum of the generalized eigenspaces of $G(\infty, \zeta)$ corresponding to eigenvalues $\mu$ with $\pm \Re \mu>0$. 
By Proposition 5.1 the spaces $\mathbb{F}_{ \pm}(\zeta)$ each have dimension $2 n$ and

$$
\mathbb{C}^{4 n}=\mathbb{F}_{+}(\zeta) \oplus \mathbb{F}_{-}(\zeta),
$$

but the projections are generally not locally uniformly bounded with respect to $\zeta$. Indeed, if the basepoint $X_{0}=(\hat{\hat{\zeta}}, 0)$ in Proposition 6.1 is such that $\hat{H}_{B}\left(X_{0}\right)$ has one or more glancing blocks, the projections do blow up near $X_{0}$.

$\mathbb{F}_{ \pm}(\zeta)$ do not vary continuously near $\zeta=0$, so it is better to write $\mathbb{F}_{ \pm}(\hat{\zeta}, \rho)$, where $\hat{\zeta} \in S^{d}$ and (initially) $\rho>0$. In [Z1] it is shown that these spaces vary smoothly (even analytically) in $\{\rho>0, \hat{\gamma}>0\}$. In addition, they extend continuously to $\{\rho \geq 0, \hat{\gamma} \geq 0\}$ (this can be seen by arguing as in [CP], Chapter 7). For $\rho>0$, $\mathbb{F}_{ \pm}(\hat{\zeta}, \rho)$ are the spaces of boundary values at $x=0$ of growing (resp. decaying) solutions of

$$
U_{x}-G(\infty, \zeta) U=0 \text { on } x \geq 0 .
$$

For $T, T_{1}, T_{2}$ as defined earlier in this section, set $\mathcal{T}=T T_{1} T_{2}$ and observe that the block form (6.8) of $G_{B}(\infty)=\mathcal{T}^{-1} G(\infty) \mathcal{T}$ corresponds to a partition of the vectors $U=\mathcal{T}^{-1} V=\left(u_{+}, u_{-}, u_{1}, \ldots, u_{p}\right)$. Denote by $\alpha_{j}$ the number of eigenvalues of $Q_{j}$ with $\Re \mu<0$ for $\hat{\gamma}>0$ (or $\rho>0$ ), and write

$$
u_{j}=\left(u_{j-}, u_{j+}\right)
$$

where $u_{j-}$ consists of the first $\alpha_{j}$ components of $u_{j}$.

Next set

$$
\begin{aligned}
& U_{P+}=\left(u_{+}, 0,0, \ldots, 0\right), \\
& U_{P-}=\left(0, u_{-}, 0, \ldots, 0\right), \\
& U_{H+}=\left(0,0,\left(0, u_{1+}\right), \ldots,\left(0, u_{p+}\right)\right), \\
& U_{H-}=\left(0,0,\left(u_{1-}, 0\right), \ldots,\left(u_{p-}, 0\right)\right),
\end{aligned}
$$

and write

$$
\begin{aligned}
& U=U_{P_{+}}+U_{P_{-}}+U_{H_{+}}+U_{H_{-}}, \\
& U_{ \pm}=U_{P_{ \pm}}+U_{H_{ \pm}}, \\
& U_{P}=U_{P_{+}}+U_{P_{-},} \\
& U_{H}=U_{H_{+}}+U_{H_{-}} .
\end{aligned}
$$

Corresponding to (6.15) we have the decomposition

$$
\mathbb{C}^{4 n}=E_{P_{+}} \oplus E_{P_{-}} \oplus E_{H_{+}} \oplus E_{H_{-}},
$$

where $E_{P+}$ is the subspace of all vectors of the form $\left(u_{+}, 0,0, \ldots, 0\right)$, etc.

Proposition 5.1 shows these subspaces have dimensions

$$
\begin{aligned}
& \operatorname{dim} E_{P_{+}}=k+l=n-1, \\
& \operatorname{dim} E_{P_{-}}=(n-k)+(n-l)=n+1, \\
& \operatorname{dim} E_{H_{+}}=(n-k)+(n-l)=n+1, \\
& \operatorname{dim} E_{H_{-}}=k+l=n-1 .
\end{aligned}
$$

Applying $\mathcal{T}(\hat{\zeta}, \rho)$ to 6.17), we obtain the smooth decomposition with uniformly bounded projections

$$
\mathbb{C}^{4 n}=F_{P_{+}}(\hat{\zeta}, \rho) \oplus F_{P_{-}}(\hat{\zeta}, \rho) \oplus F_{H_{+}}(\hat{\zeta}, \rho) \oplus F_{H_{-}}(\hat{\zeta}, \rho),
$$

where $F_{P+}=\mathcal{T}(\hat{\zeta}, \rho) E_{P+}$, etc. 
Recall that $\mathcal{T}$ is $C^{\infty}$ and defined locally near some basepoint $X_{0}=(\hat{\hat{\zeta}}, 0) \in$ $S_{+}^{d} \times \overline{\mathbb{R}}_{+}$. Block by block analysis (see $[\mathrm{CP}$, Chapter 7, Remark 3.6) using the special form (6.11) of $\mathcal{Q}\left(X_{0}\right)$ shows that

$$
F_{P-}\left(X_{0}\right) \oplus F_{H_{-}}\left(X_{0}\right)=\mathbb{F}_{-}\left(X_{0}\right)
$$

where $\mathbb{F}_{-}(\hat{\zeta}, \rho)$ is the continuous extension to $S_{+}^{d} \times \overline{\mathbb{R}}_{+}$of the space introduced in Definition 6.2

Remark 6.2. (1) We stress that the analogue of (6.20) is not true for $\mathbb{F}_{+}\left(X_{0}\right)$. It is clear that such a property would be inconsistent with the fact that the projections in (6.19) are uniformly bounded near $X_{0}$, while those in (6.13) are generally not.

(2) If the basepoint $X_{0}$ is such that none of the blocks $\mathcal{Q}_{k}\left(X_{0}\right)$ in (6.10) are glancing, then (6.20) remains true for $(\hat{\zeta}, \rho)$ near $X_{0}$. Otherwise (6.20) is not necessarily true for nearby points distinct from $X_{0}$.

More precisely, we can decompose $F_{H-}(\hat{\zeta}, \rho)$ by blocks in the obvious way

$$
F_{H-}(\hat{\zeta}, \rho)=\bigoplus_{j=1}^{p} F_{H_{j-}}(\hat{\zeta}, \rho) .
$$

Here

$$
F_{H_{j-}}(\hat{\zeta}, \rho)=\mathcal{T}(\hat{\zeta}, \rho) E_{H_{j-}},
$$

where $E_{H_{j-}}$ is the subspace of $E_{H_{-}}$consisting of all vectors of the form $(0,0,0, \ldots$, $\left.\left(u_{j-}, 0\right), 0, \ldots\right)$. We have

$$
F_{H_{j-}}\left(X_{0}\right) \subset \mathbb{F}_{-}\left(X_{0}\right),
$$

but the same is not necessarily true for nearby points different from $X_{0}$ when the $\mathcal{Q}_{j}$ block is glancing.

In constructing degenerate symmetrizers we sometimes need a decomposition for which properties like (6.23) hold in a full neighborhood of the basepoint. For this we need a further conjugation, this time by a matrix $T_{3}(\hat{\zeta}, \rho)$ that is generally not $C^{\infty}$ but merely continuous. The following lemma is essentially Lemma 2.6 of $[\mathrm{K}]$.

Lemma 6.2. Let $\mathcal{Q}_{k}$ in (6.10) be a glancing block of size $\nu_{k}$. There exists a unitary matrix $T_{\mathcal{Q}_{k}}(\hat{\zeta}, \rho)$, defined in a neighborhood of $X_{0}$ and with uniformly bounded inverse, such that $T_{\mathcal{Q}_{k}}\left(X_{0}\right)=I$ and

$$
T_{\mathcal{Q}_{k}}^{-1} \mathcal{Q}_{k} T_{\mathcal{Q}_{k}}(\hat{\zeta}, \rho)=\left(\begin{array}{ccccc}
\kappa_{k 1} & * & \cdots & * & * \\
0 & \kappa_{k 2} & * & \cdots & * \\
\vdots & \vdots & \ddots & \ddots & \vdots \\
0 & \cdots & \cdots & \kappa_{k \nu_{k}-1} & * \\
0 & \cdots & \cdots & 0 & \kappa_{k \nu_{k}}
\end{array}\right) .
$$

$T_{\mathcal{Q}_{k}}$ can be chosen so that for $\hat{\gamma}>0$ the eigenvalues $\kappa_{k j}$ with $\Re \kappa_{k j}<0$ stand in the first rows. $T_{\mathcal{Q}_{k}}$ is $C^{\infty}$ in $\rho>0$ but just continuous up to $\rho=0$.

Definition 6.3. Define $T_{3}$ to be a block diagonal matrix with the same number and size of blocks as $G_{B}(\infty)$, where each glancing block $\mathcal{Q}_{k}$ of $G_{B}$ is replaced by $T_{\mathcal{Q}_{k}}$ and each nonglancing block of $G_{B}$ is replaced by an identity matrix of the same size. 
Using $T_{3}$, we obtain a slight modification of the decomposition (6.17)

$$
\begin{aligned}
& \mathbb{C}^{4 n}=E_{P_{+}} \oplus E_{P_{-}} \oplus E_{H_{+, c}}(\hat{\zeta}, \rho) \oplus E_{H_{-, c}}(\hat{\zeta}, \rho), \\
& U=U_{P_{+}}+U_{P_{-}}+U_{H_{+, c}}+U_{H_{-, c}},
\end{aligned}
$$

where $E_{H_{ \pm, c}}(\hat{\zeta}, \rho)=T_{3}(\hat{\zeta}, \rho) E_{H_{ \pm}}$. The subscript "c" is a reminder that $T_{3}$ is merely continuous up to $\rho=0$.

Apply $\mathcal{T}=T T_{1} T_{2}$ to this decomposition to obtain a continuous decomposition near 6.19)

$$
\mathbb{C}^{4 n}=F_{P_{+}}(\hat{\zeta}, \rho) \oplus F_{P_{-}}(\hat{\zeta}, \rho) \oplus F_{H_{+, c}}(\hat{\zeta}, \rho) \oplus F_{H_{-, c}}(\hat{\zeta}, \rho)
$$

where $F_{H_{ \pm, c}}(\hat{\zeta}, \rho)=\mathcal{T}(\hat{\zeta}, \rho) E_{H_{ \pm, c}}(\hat{\zeta}, \rho)$. Here again the projections are uniformly bounded.

In place of (6.20) we now have

$$
F_{P-}(\hat{\zeta}, \rho) \oplus F_{H_{-, c}}(\hat{\zeta}, \rho)=\mathbb{F}_{-}(\hat{\zeta}, \rho)
$$

for $(\hat{\zeta}, \rho)$ in a neighborhood of $X_{0}$.

Corresponding to (6.23) we have

$$
F_{H_{j-, c}}(\hat{\zeta}, \rho) \subset \mathbb{F}_{-}(\hat{\zeta}, \rho)
$$

near $X_{0}$, where

$$
F_{H_{j-, c}}(\hat{\zeta}, \rho)=\mathcal{T}(\hat{\zeta}, \rho) T_{3}(\hat{\zeta}, \rho) E_{H_{j-}} .
$$

Definition 6.4. Let $E_{H_{j-}}$ be as in (6.22) and similarly define $E_{H_{j+}}$. Denote the images of these spaces under $T_{3}(\hat{\zeta}, \rho)$ by $E_{H_{j-, c}}$ and $E_{H_{j+, c}}$, respectively. Thus, we have decompositions

$$
\begin{gathered}
E_{H_{ \pm}}=\bigoplus_{j=1}^{p} E_{H_{j \pm}}, \\
E_{H_{ \pm, c}}=\bigoplus_{j=1}^{p} E_{H_{j \pm, c}}, \\
E_{H_{j}}=E_{H_{j+}} \oplus E_{H_{j-}}=E_{H_{j+, c}}(\hat{\zeta}, \rho) \oplus E_{H_{j-, c}}(\hat{\zeta}, \rho),
\end{gathered}
$$

and with obvious notation

$$
\begin{aligned}
& U_{H_{j}}=U_{H_{j+}}+U_{H_{j-}}, \\
& U_{H_{j}}=U_{H_{j+, c}}+U_{H_{j-, c}} .
\end{aligned}
$$

6.3. Standard symmetrizers. The $n \times n$ second order initial value problem we begin with leads to a $4 n \times 4 n$ doubled first order boundary problem on $x \geq 0$.

Recall the doubled boundary problem (5.1)

$$
\begin{aligned}
& U_{x}-G(x, \lambda, \eta) U=F, \\
& \Gamma U=0 \text { on } x=0,
\end{aligned}
$$

where $\Gamma$ is a $2 n \times 4 n$ matrix giving the doubled boundary conditions. 
MZ conjugation using $W(x, \zeta)$ (Lemma [5.1) transforms $G(x, \zeta)$ to the constant coefficient matrix $G(\infty, \zeta)$, and further conjugation using $\mathcal{T}=T T_{1} T_{2}$ leads to $G_{B}(\infty)$ as in Proposition 6.1 In place of (6.33) we must now study

$$
\begin{gathered}
U_{x}-G_{B}(\infty, \hat{\zeta}, \rho) U=F, \\
\Gamma_{1}(\hat{\zeta}, \rho) U=0 \text { on } x=0,
\end{gathered}
$$

where $\Gamma_{1}=\Gamma W \mathcal{T}$.

Here we wish to illustrate the use of Kreiss symmetrizers to prove estimates (especially in the low frequency region) in a simpler situation where $\Gamma_{1}$ is replaced by an artificial boundary condition $\Gamma_{a}$ that satisfies the uniform Lopatinski condition near the basepoint $X_{0}$.

Let $\mathbb{F}_{-}(\hat{\zeta}, \rho)$ be the $2 n$-dimensional continuous extension of the decaying generalized eigenspace for $G(\infty, \zeta)$ defined before, and set

$$
\mathbb{E}_{-, c}(\hat{\zeta}, \rho)=\mathcal{T}^{-1} \mathbb{F}_{-}
$$

which is the same as $E_{P_{-}} \oplus E_{H_{-, c}}(\hat{\zeta}, \rho)$ in the notation of (6.25)).

Definition 6.5. A boundary operator $\Gamma_{a}(\hat{\zeta}, \rho)$ depending continuously on $(\hat{\zeta}, \rho)$ is said to satisfy the uniform Lopatinski condition at $X_{0}=(\underline{\hat{\zeta}}, 0) \in S_{+}^{d} \times \overline{\mathbb{R}}_{+}$if there exists $C>0$ such that

$$
\left|\Gamma_{a}\left(X_{0}\right) u\right| \geq C|u|
$$

for $u \in \mathbb{E}_{-, c}\left(X_{0}\right)$.

Remark 6.3. (1) By continuity of $\Gamma_{a}$ and $\mathbb{E}_{-, c}$, if $\Gamma_{a}$ satisfies the uniform Lopatinski condition at $X_{0}$, it satisfies (6.36) uniformly in a neighborhood of $X_{0}$.

(2) Let

$$
\mathbb{E}_{-}=E_{P_{-}} \oplus E_{H_{-}}
$$

the summands on the right being as in (6.17). Since $T_{3}\left(X_{0}\right)=I, T_{3}$ is continuous at $X_{0}$, and $\mathbb{E}_{-, c}=T_{3} \mathbb{E}_{-}$, it follows that if $\Gamma_{a}$ satisfies the uniform Lopatinski condition at $X_{0}$, we also have

$$
\left|\Gamma_{a}(\hat{\zeta}, \rho) u\right| \geq C|u|
$$

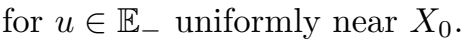

(3) $\Gamma_{1}$ in 6.34 fails to satisfy the uniform Lopatinski condition at $X_{0}$ (see Corollary 7.1). This degeneracy forces us to be careful in Part 2 (Lemma 7.1 e.g.) about the distinction between $\mathbb{E}_{-, c}$ and $\mathbb{E}_{-}$, since it prevents us from simply arguing by continuity as above to justify interchanging these two spaces.

The symmetrizer for the problem

$$
\begin{aligned}
& U_{x}-G_{B}(\infty) U=F, \\
& \Gamma_{a} U=g \text { on } x=0
\end{aligned}
$$


is a $4 n \times 4 n$ matrix constructed by blocks in a neighborhood of $X_{0}$

$$
S(\hat{\zeta}, \rho)=\left(\begin{array}{ccccc}
S_{+}(\zeta) & & & & \\
& S_{-}(\zeta) & & & \\
& & S_{1}(\hat{\zeta}, \rho) & & \\
& & & \ddots & \\
& & & & S_{p}(\hat{\zeta}, \rho)
\end{array}\right),
$$

where the $S_{ \pm}, S_{j}$ are $C^{\infty}$ functions of their arguments. We will sometimes write

$$
S=\left(\begin{array}{ll}
S_{P} & \\
& S_{H}
\end{array}\right),
$$

where in fact $S_{P}$ can be taken to be simply

$$
S_{P}=\left(\begin{array}{cc}
C I & \\
& -I
\end{array}\right)
$$

for some large $C>0$.

In the following discussion $U=U(x, \zeta),\langle$,$\rangle denotes the inner product in \mathbb{C}^{4 n}$,

$$
(U(x, \zeta), V(x, \zeta)) \equiv \int_{0}^{\infty}\langle U(x, \zeta), V(x, \zeta)\rangle d x
$$

and

$$
\begin{aligned}
& |U|_{2}=|U(x, \zeta)|_{L^{2}(x)}, \\
& |U|=|U(0, \zeta)| .
\end{aligned}
$$

In [MZ1] the $S_{j}$ are constructed so that $S=S^{*}$, with interior estimates

$$
\begin{aligned}
& \left(\operatorname{Re} S G_{B}(\infty) U_{P}, U_{P}\right) \geq C\left|U_{P}\right|_{2}^{2}, \\
& \left(\operatorname{Re} S G_{B}(\infty) U_{H_{j}}, U_{H_{j}}\right) \geq\left(\gamma+\rho^{2}\right)\left|U_{H_{j}}\right|_{2}^{2},
\end{aligned}
$$

as well as boundary estimates

$$
\begin{array}{ll}
\text { (a) } & \left(S U_{P}, U_{P}\right) \geq C\left|U_{P_{+}}\right|^{2}-\left|U_{P_{-}}\right|^{2}, \\
\text { (b) } \quad\left(S U_{H_{j}}, U_{H_{j}}\right) \geq C\left|U_{H_{j+}}\right|^{2}-\left|U_{H_{j-}}\right|^{2},
\end{array}
$$

both holding uniformly near the basepoint $X_{0}$.

Assuming $\Gamma_{a}$ satisfies the uniform Lopatinski condition at $X_{0}$, we have

$$
\left|U_{-}\right|^{2} \leq C\left|\Gamma_{a} U_{-}\right|^{2} \leq C\left(\left|\Gamma_{a} U\right|^{2}+\left|U_{+}\right|^{2}\right)
$$

at $X_{0}$ and in fact uniformly near $X_{0}$ in view of Remark 6.3(2).

Using the previous two estimates, we obtain

$$
\begin{aligned}
& (S U, U) \geq C\left|U_{+}\right|^{2}-\left|U_{-}\right|^{2}=C\left|U_{+}\right|^{2}+\left|U_{-}\right|^{2}-2\left|U_{-}\right|^{2} \\
& \geq C\left|U_{+}\right|^{2}+\left|U_{-}\right|^{2}-C_{1}\left(\left|\Gamma_{a} U\right|^{2}+\left|U_{+}\right|^{2}\right) \\
& \geq C_{2}\left|U_{+}\right|^{2}+\left|U_{-}\right|^{2}-C_{1}\left|\Gamma_{a} U\right|^{2},
\end{aligned}
$$

provided $C$ was big enough.

From (6.45), (6.48), and the identity

$$
-\langle S U(0), U(0)\rangle=\int_{0}^{\infty} \partial_{x}\langle S U, U\rangle d x=\left(2 \Re S G_{B} U, U\right)+2 \Re(S F, U),
$$


we obtain the [MZ1]-type estimate

$$
\begin{aligned}
& \left(\left|U_{P}\right|_{2}^{2}+\left(\gamma+\rho^{2}\right)\left|U_{H}\right|_{2}^{2}\right)+|U|^{2} \\
& \quad \leq C\left(\left|F_{P}\right|_{2}^{2}+\frac{1}{\left(\gamma+\rho^{2}\right)}\left|F_{H}\right|_{2}^{2}\right)+C\left|\Gamma_{a} U\right|^{2},
\end{aligned}
$$

uniformly near $X_{0}$. Here we have used

$$
|(S F, U)| \leq\left(C_{\delta}\left|F_{P}\right|_{2}^{2}+\delta\left|U_{P}\right|_{2}^{2}\right)+\left(\frac{C_{\delta}}{\left(\gamma+\rho^{2}\right)}\left|F_{H}\right|_{2}^{2}+\delta\left(\gamma+\rho^{2}\right)\left|U_{H}\right|_{2}^{2}\right) .
$$

\section{EVANS FUNCTION FOR THE DOUbled BOUNDARY PROBLEM}

In this section we will show how the first order vanishing of the Evans function $\mathcal{D}(\zeta)$ for the $2 n \times 2 n$ system (2.19) on $\mathbb{R}$ implies a degenerate Lopatinski condition for the $4 n \times 4 n$ doubled boundary problem (5.1)

$$
\begin{aligned}
& U_{x}-G(x, \zeta) U=F, \\
& \Gamma U=0 \text { on } x=0,
\end{aligned}
$$

where $U=\left(U_{+}, U_{-}\right)$and $\Gamma U=U_{+}-U_{-}$. We should mention that whenever $U$ represents a solution to (7.1), the notation $U_{ \pm}$is that of (5.2), so

$$
U_{+}=\left(U_{1}, \ldots, U_{2 n}\right), \quad U_{-}=\left(U_{2 n+1}, \ldots, U_{4 n}\right) .
$$

The $U_{ \pm}$notation of 6.16 is reserved for solutions $U$ to the problem $\left(\partial_{x}-G_{B}\right)$.

The Evans function for (7.1), $\mathbb{D}(\hat{\zeta}, \rho)$, is a determinant that measures the degree of linear dependency between two $2 n$-dimensional subspaces of $\mathbb{C}^{4 n}$, namely, $\operatorname{ker} \Gamma$ and $\mathcal{E}_{-}(\hat{\zeta}, \rho)$. The latter space is defined for $\hat{\gamma}>0, \rho>0$ as the space of boundary values at $x=0$ of decaying solutions to the homogeneous problem

$$
U_{x}-G(x, \zeta) U=0 .
$$

Like $\mathbb{F}_{-}(\hat{\zeta}, \rho)$ (recall Definition 6.2), $\mathcal{E}_{-}(\hat{\zeta}, \rho)$ has a continuous extension to $\hat{\gamma} \geq 0$, $\rho \geq 0$. In fact it is easy to check that we have

$$
\mathcal{E}_{-}(\hat{\zeta}, \rho)=W(0, \hat{\zeta}, \rho) \mathbb{F}_{-}(\hat{\zeta}, \rho),
$$

where $W$ is the MZ conjugator of Lemma 5.1 .

Remark 7.1. The individual functions $\mathcal{U}_{j}^{R, L}(x, \widehat{\zeta}, \rho)$ appearing in the definition of $\mathcal{D}(\zeta)$ are locally analytic in $(\widehat{\zeta}, \rho)$ on $\{\widehat{\gamma}>0, \rho>0\}$. This is a consequence of a standard contraction mapping argument $\mathrm{Co}$ together with the corresponding fact for solutions to the systems obtained from (2.19) by taking limits as $x \rightarrow \pm \infty$. This argument also shows that the individual solutions corresponding to fast decaying modes extend analytically to $\{\widehat{\gamma} \geq 0, \rho \geq 0\}$. The fast decaying solutions are independent of $\widehat{\zeta}$ at $\rho=0$ and so extend smoothly as functions of $\zeta$ as well.

Since the subspace $\mathcal{E}_{-}(\widehat{\zeta}, \rho)$ has a continuous extension to $\{\widehat{\gamma} \geq 0, \rho \geq 0\}$, we can if necessary redefine the individual solutions $\mathcal{U}_{j}^{R, L}$ corresponding to slowly decaying modes so that they have continuous extensions to $\widehat{\gamma^{\prime}} \geq 0, \rho \geq 0$. Henceforth, we assume this has been done.

Using Notation 1.1, we set for $x \geq 0$

$$
\begin{aligned}
& \mathcal{U}_{j+}^{R}(x, \hat{\zeta}, \rho)=\mathcal{U}_{j}^{R}(x, \hat{\zeta}, \rho), \\
& \mathcal{U}_{j-}^{L}(x, \hat{\zeta}, \rho)=\mathcal{U}_{j}^{L}(-x, \hat{\zeta}, \rho)
\end{aligned}
$$


Let $\phi(x)$ as before be the derivative of the profile $\psi$. Let $e_{1} \in \mathbb{C}^{2 n}$ be the unit vector

$$
e_{1}=\frac{(\phi(0), 0)}{|\phi(0)|}
$$

and extend to an orthonormal basis $e_{1}, \ldots, e_{2 n}$ of $\mathbb{C}^{2 n}$.

Definition 7.1. (1) Define the Evans function for the doubled boundary problem $\left(\partial_{x}-G, \Gamma\right)(17.1)$ as the $4 n \times 4 n$ determinant

$$
\mathbb{D}(\hat{\zeta}, \rho)=\left.\operatorname{det}\left(\begin{array}{ccccccccc}
e_{1} & \cdots & e_{2 n} & \mathcal{U}_{1+}^{R} & \cdots & \mathcal{U}_{n+}^{R} & 0 & \cdots & 0 \\
e_{1} & \cdots & e_{2 n} & 0 & \cdots & 0 & \mathcal{U}_{1-}^{L} & \cdots & \mathcal{U}_{n-}^{L}
\end{array}\right)\right|_{x=0} .
$$

(2) Recalling the normalization (2.25), we set

$$
\mathcal{E}_{-, \phi}(\hat{\zeta}, \rho)=\left.\operatorname{span}\left(\begin{array}{l}
\mathcal{U}_{1+}^{R} \\
\mathcal{U}_{n-}^{L}
\end{array}\right)\right|_{(0, \hat{\zeta}, \rho)} .
$$

For $\epsilon>0$ fixed denote by $\mathcal{E}_{-, \phi, \epsilon}^{c}(\hat{\zeta}, \rho)$ any complementary subspace in $\mathcal{E}_{-}(\hat{\zeta}, \rho)$ varying continuously with $(\hat{\zeta}, \rho)$ such that

$$
\mathcal{E}_{-}(\hat{\zeta}, \rho)=\mathcal{E}_{-, \phi}(\hat{\zeta}, \rho) \oplus \mathcal{E}_{-, \phi, \epsilon}^{c}(\hat{\zeta}, \rho)
$$

with uniformly bounded projections for $0 \leq \rho \leq \epsilon$.

Proposition 7.1. (1) Let $\mathcal{D}(\hat{\zeta}, \rho)$ be the Evans function defined in Lemma [2.1. Then

$$
\mathcal{D}(\hat{\zeta}, \rho)=(-1)^{n} \mathbb{D}(\hat{\zeta}, \rho) .
$$

(2) Under the Evans assumption (H4), we have the following.

(a) For any choice of $0<\delta<R$ there is a constant $C_{\delta, R}$ such that when $\delta \leq$ $\rho \leq R$,

$$
|\Gamma u| \geq C_{\delta, R}|u| \text { for } u \in \mathcal{E}_{-}(\hat{\zeta}, \rho) .
$$

(b) There exist positive constants $C_{1}, C_{2}, \delta$ such that

$$
C_{1} \rho|u| \leq|\Gamma u| \leq C_{2} \rho|u| \text { for } u \in \mathcal{E}_{-, \phi}(\hat{\zeta}, \rho)
$$

for $0 \leq \rho \leq \delta$.

(c) For $\mathcal{E}_{-, \phi, \epsilon}^{c}(\hat{\zeta}, \rho)$ as in (7.8) there exists $C>0$ such that

$$
|\Gamma u| \geq C|u| \text { for } u \in \mathcal{E}_{-, \phi, \epsilon}^{c}(\hat{\zeta}, \rho)
$$

for $0 \leq \rho \leq \epsilon$.

(d) For any choice of $R>0$ there is a constant $C_{R}$ such that for $0 \leq \rho \leq R$,

$$
|\Gamma u| \geq C_{R} \rho|u| \text { for } u \in \mathcal{E}_{-}(\hat{\zeta}, \rho) .
$$

Proof. (1) Let us denote the matrix in (77.6) by $\mathcal{M}$. Perform the row operation of subtracting the first row of $\mathcal{M}$ (which has $2 n$ components) from the second to see (7.9).

(2)(a) The assumption (H4) implies $\Gamma u$ is nonvanishing for nonzero $u \in \mathcal{E}_{-}(\hat{\zeta}, \rho)$ when $\rho>0$. The existence of $C_{\delta, R}$ thus follows by continuity and compactness. 
(2)(b) Perform a column operation to replace the last column of $\mathcal{M}$ by $\left(\begin{array}{l}\mathcal{U}_{1+}^{R} \\ \mathcal{U}_{n-}^{L}\end{array}\right)$, and call the resulting matrix $\mathcal{M}_{1}$. Observe that since these fast modes depend analytically on $\rho$, we have

$$
\left(\begin{array}{l}
\mathcal{U}_{1+}^{R} \\
\mathcal{U}_{n-}^{L}
\end{array}\right)(0, \hat{\zeta}, \rho)=\left(\begin{array}{l}
(\phi(0), 0) \\
(\phi(0), 0)
\end{array}\right)+\left(\begin{array}{l}
c_{1}(\hat{\zeta}) \\
c_{2}(\hat{\zeta})
\end{array}\right) \rho+O\left(\rho^{2}\right) .
$$

Recall the definition of $e_{1}$ and use linearity of the determinant in the last column to see that if the coefficient $c_{1}-c_{2}$ were to vanish for some $\hat{\zeta}$, then (H4) would be violated. Since

$$
\Gamma\left(\begin{array}{l}
\mathcal{U}_{1+}^{R} \\
\mathcal{U}_{n-}^{L}
\end{array}\right)=\mathcal{U}_{1+}^{R}-\mathcal{U}_{n-}^{L},
$$

this gives (7.11).

(2)(c) Let $v_{1}(\hat{\zeta}, \rho), \ldots, v_{2 n}(\hat{\zeta}, \rho)$ be the last $2 n$ columns of the matrix $\mathcal{M}_{1}$ defined above. These vectors form a basis for $\mathcal{E}_{-}(\hat{\zeta}, \rho)$. Any vector in $\mathcal{E}_{-, \phi, \epsilon}^{c}(\hat{\zeta}, \rho)$ has the form of a linear combination with coefficients depending continuously on $(\hat{\zeta}, \rho)$

$$
w=\sum_{j=1}^{2 n} c_{j, \epsilon}(\hat{\zeta}, \rho) v_{j} .
$$

Set $c_{\epsilon}^{\prime}=\left(c_{1, \epsilon}, \ldots, c_{2 n-1, \epsilon}\right)$. The condition that the projections in (7.8) are uniformly bounded implies there is an $\epsilon_{0}>0$ such that

$$
\left|c_{\epsilon}^{\prime}(\hat{\zeta}, \rho)\right| \geq \epsilon_{0}\left|c_{2 n, \epsilon}(\hat{\zeta}, \rho)\right|
$$

for $0 \leq \rho \leq \epsilon$.

In view of (H5) we just need to show that $\Gamma w$ is nonvanishing at $\rho=0$ for $w$ as in (7.16), (7.17) with $\left|\left(c_{\epsilon}^{\prime}, c_{2 n, \epsilon}\right)\right|=1$, since (7.12) then follows by continuity and compactness. Suppose $\Gamma w=0$ at $(\hat{\zeta}, 0)$ for some such $w$. Because of (7.17) some $c_{j, \epsilon}$ with $j \leq 2 n-1$, say $c_{1, \epsilon}$, satisfies

$$
\left|c_{1, \epsilon}(\hat{\zeta}, \rho)\right|^{2} \geq \frac{1}{2} \cdot \frac{\min \left(\frac{1}{2}, \frac{\epsilon_{0}^{2}}{2}\right)}{2 n-1}
$$

for $\rho$ near 0 . Since $\Gamma w=0$ at $\rho=0$ and $w(\hat{\zeta}, \rho)$ is continuous, we have

$$
w(\hat{\zeta}, \rho)=\left(\begin{array}{l}
a \\
a
\end{array}\right)(\hat{\zeta})+o(1) .
$$

Write $v_{j}=\left(v_{j+}, v_{j-}\right)$, use column operations to replace $v_{1}$ in $\mathcal{M}_{1}$ by $w$, and call the resulting matrix $\mathcal{M}_{2}=$

$$
\left(\begin{array}{llllllll}
e_{1} & \cdots & e_{2 n} & a(\hat{\zeta})+o(1) & v_{2+} & \cdots & v_{2 n-1,+} & (\phi(0), 0)+O(\rho) \\
e_{1} & \cdots & e_{2 n} & a(\hat{\zeta})+o(1) & v_{2-} & \cdots & v_{2 n-1,-} & (\phi(0), 0)+O(\rho)
\end{array}\right) .
$$

(7.18) implies $\left|\operatorname{det} \mathcal{M}_{2}(\hat{\zeta}, \rho)\right| \geq C\left|\operatorname{det} \mathcal{M}_{1}(\hat{\zeta}, \rho)\right|$ for some $C>0$ uniformly near $(\hat{\zeta}, 0)$. But

$$
\operatorname{det} \mathcal{M}_{2}(\hat{\zeta}, \rho)=O(\rho) o(1) \text { as } \rho \rightarrow 0 .
$$

This contradicts the assumed vanishing of $\operatorname{det} \mathcal{M}=\operatorname{det} \mathcal{M}_{1}$ to exactly first order at $\rho=0$. 
(2)(d) For any fixed $(\hat{\zeta}, \rho)$ let $u^{*}=\left(\begin{array}{l}u_{+}(\hat{\zeta}, \rho) \\ u_{-}(\hat{\zeta}, \rho)\end{array}\right) \in \mathcal{E}_{-}(\hat{\zeta}, \rho)$ be an element where the minimum

$$
\min _{|u|=1, u \in \mathcal{E}_{-}(\hat{\zeta}, \rho)}|\Gamma u|
$$

is attained. At the cost of modifying $\mathbb{D}(\hat{\zeta}, \rho)$ by a nonvanishing factor $\alpha(\hat{\zeta}, \rho)$ of size $\sim 1$, we can redefine the last $2 n$ columns of $\mathcal{M}$ so that $u^{*}$ appears in (say) the $(2 n+1)$ st column of $\mathcal{M}$. Next perform column operations to replace $u^{*}$ by

$$
\left(\begin{array}{c}
u_{+}(\hat{\zeta}, \rho)-u_{-}(\hat{\zeta}, \rho) \\
0
\end{array}\right)=\left(\begin{array}{c}
\Gamma u^{*} \\
0
\end{array}\right) .
$$

This shows that $|\mathbb{D}(\hat{\zeta}, \rho)| \leq C\left|\Gamma u^{*}\right|$, so (H4) implies the result.

Next we need to rephrase these estimates in terms of the coordinates for the problem $\left(\partial_{x}-G_{B}, \Gamma_{1}\right)$ (6.30). Recall the decompositions of $U \in \mathbb{C}^{4 n}$ given in (6.16) and (6.25)

$$
\begin{aligned}
& U=U_{P_{+}}+U_{P_{-}}+U_{H_{+}}+U_{H_{-}}, \\
& U=U_{P_{+}}+U_{P_{-}}+U_{H_{+}, c}+U_{H_{-, c}},
\end{aligned}
$$

and set

$$
\begin{aligned}
& U_{-}=U_{P_{-}}+U_{H_{-}} \in \mathbb{E}_{-}, \\
& U_{-, c}=U_{P_{-}}+U_{H_{-, c}} \in \mathbb{E}_{-, c}(\hat{\zeta}, \rho) .
\end{aligned}
$$

Define the one-dimensional subspace $E_{P_{1-}}(\hat{\zeta}, \rho)$ of $E_{P-}$ by

$$
\mathcal{E}_{-, \phi}=W \mathcal{T} E_{P_{1-}},
$$

and for $\epsilon>0$ fixed, choose a smoothly varying complementary subspace $E_{P_{2-, \epsilon}}$ such that

$$
\begin{aligned}
& E_{P_{-}}=E_{P_{1-}}(\hat{\zeta}, \rho) \oplus E_{P_{2-, \epsilon}}(\hat{\zeta}, \rho), \\
& U_{P_{-}}=U_{P_{1-}}+U_{P_{2-, \epsilon}}
\end{aligned}
$$

with uniformly bounded projections for $0 \leq \rho \leq \epsilon$. Then

$$
\mathcal{E}_{-, \phi, \epsilon}^{c} \equiv W \mathcal{T}\left(E_{P_{2-, \epsilon}} \oplus E_{H_{-, c}}\right)
$$

is a choice that works in (7.8).

The next corollary is then an immediate consequence of Proposition 7.1 Here and henceforth, we will often suppress in the notation the dependence of operators and spaces on $(\hat{\zeta}, \rho)$. Recall $\Gamma_{1}=\Gamma W \mathcal{T}$.

Corollary 7.1. There exist positive constants $C_{1}, \ldots, C_{4}$ and $\delta_{0}$ such that for $0 \leq$ $\rho \leq \delta_{0}$

$$
\begin{aligned}
& \text { (a) }\left|C_{1} \rho\right| U_{P_{1,-}}|\leq| \Gamma_{1} U_{P_{1,-}}\left|\leq C_{2} \rho\right| U_{P_{1,-}} \mid \\
& \text { (b) }\left|\Gamma_{1}\left(U_{H_{-, c}}+U_{P_{2,-}, \epsilon}\right)\right| \geq C_{3}\left(\left|U_{H_{-, c}}\right|+\left|U_{P_{2,-}, \epsilon}\right|\right), \\
& \text { (c) }\left|\Gamma_{1} U_{-, c}\right| \geq C_{4} \rho\left|U_{-, c}\right| .
\end{aligned}
$$

These estimates hold uniformly near the basepoint $X_{0}$.

Part (a) of the corollary shows that $\Gamma_{1}$ fails to satisfy the uniform Lopatinski condition near $X_{0}$. The following lemma, which gives a more precise version of (7.29) (c), is essential for the construction of degenerate symmetrizers. 
Lemma 7.1. There exists a constant $\delta>0$ such that for $\rho$ sufficiently small we have

$$
\left|\Gamma_{1} U_{-, c}\right| \geq \delta\left(\left|U_{H_{-, c}}\right|+\rho\left|U_{P_{-}}\right|\right)
$$

uniformly near $X_{0}$.

Proof. We will deduce (7.30) from the stronger inequality (7.31) below. We have in view of (7.29) (a), (b)

$$
\begin{gathered}
\left|\Gamma_{1} U_{-, c}\right|=\left|\Gamma_{1} U_{H_{-, c}}+\Gamma_{1} U_{P_{1,-}}+\Gamma_{1} U_{P_{2,-}, \epsilon}\right| \\
\geq C\left(\left|U_{H_{-, c}}\right|+\left|U_{P_{2,-}, \epsilon}\right|\right)-C \rho\left|U_{P_{1,-}}\right| .
\end{gathered}
$$

Adding a sufficiently small multiple of this inequality to the inequality (7.29) (c)

$$
\left|\Gamma_{1} U_{-, c}\right| \geq C \rho\left|U_{-, c}\right|=C \rho\left(\left|U_{H_{-, c}}\right|+\left|U_{P_{1,-}}\right|+\left|U_{P_{2,-},}\right|\right),
$$

we obtain for $\rho$ small

$$
\left|\Gamma_{1} U_{-, c}\right| \geq \delta\left(\left|U_{H_{-, c}}\right|+\rho\left|U_{P_{1,-}}\right|+\left|U_{P_{2,-}, \epsilon}\right|\right),
$$

which implies (7.30).

\section{Part 3. Zero mass perturbations}

\section{Degenerate Symmetrizer For SMall Frequencies}

We are now in a position to construct a degenerate symmetrizer for the problem

$$
\begin{array}{r}
U_{x}-G_{B}(\infty, \zeta) U=F, \\
\Gamma_{1} U=g \text { on } x=0,
\end{array}
$$

where $\Gamma_{1}=\Gamma W \mathcal{T}$. We will use the same notation for pairings and norms as in the earlier discussion of the problem $\left(\partial_{x}-G_{B}, \Gamma_{a}\right)$, and we shall focus mainly on the new points.

As before we construct a symmetrizer $S=S^{*}$ of the form (6.40) for $G_{B}(\infty)$ working block by block. Let

$$
S=\left(\begin{array}{cc}
S_{P} & 0 \\
0 & S_{H}
\end{array}\right)
$$

The main difference here is that we take the $S_{P}$ block to be degenerate

$$
S_{P}=\left(\begin{array}{cc}
C I & 0 \\
0 & -\rho^{2}
\end{array}\right),
$$

where the two subblocks have sizes $n-1$ and $n+1$, respectively (recall (6.18)).

The construction of the $S_{H}$ block proceeds just as before, except that now in place of (6.46) (b) we need

$$
\left(S U_{H_{j}}, U_{H_{j}}\right) \geq C\left|U_{H_{j+,}}\right|^{2}-\left|U_{H_{j-, c}}\right|^{2}
$$

uniformly near the basepoint $X_{0}$. Here $U_{H_{j \pm, c}}$ is as in (6.32). This can be arranged by the usual procedure (see Chapter 7 in $[\mathrm{CP}]$ or $[\mathrm{K}]$ ). Summing (8.4) gives

$$
\left(S U_{H}, U_{H}\right) \geq C\left|U_{H_{+, c}}\right|^{2}-\left|U_{H_{-, c}}\right|^{2} .
$$

Thus, we obtain interior estimates

$$
\begin{aligned}
& \left(\operatorname{Re} S G_{B}(\infty) U_{P}, U_{P}\right) \geq C\left|U_{P_{+}}\right|_{2}^{2}+\rho^{2}\left|U_{P_{-}}\right|_{2}^{2}, \\
& \left(\operatorname{Re} S G_{B}(\infty) U_{H}, U_{H}\right) \geq\left(\gamma+\rho^{2}\right)\left|U_{H}\right|_{2}^{2},
\end{aligned}
$$


as well as boundary estimates

$$
\begin{aligned}
& \left(S U_{P}, U_{P}\right) \geq C\left|U_{P_{+}}\right|^{2}-\rho^{2}\left|U_{P_{-}}\right|^{2}, \\
& \left(S U_{H}, U_{H}\right) \geq C\left|U_{H_{+, c}}\right|^{2}-\left|U_{H_{-,}}\right|^{2},
\end{aligned}
$$

uniformly near $X_{0}$.

Now, Lemma 7.1 implies

$$
\left|U_{H_{-, c}}\right|^{2}+\rho^{2}\left|U_{P_{-}}\right|^{2} \leq C\left|\Gamma_{1} U_{-, c}\right|^{2} \leq C\left(\left|\Gamma_{1} U\right|^{2}+\left|U_{+, c}\right|^{2}\right),
$$

where $U_{+, c}=U_{P_{+}}+U_{H_{+, c}}$. Using (8.7) and (8.8), we obtain for $\rho$ small

$$
\begin{aligned}
& (S U, U) \geq C\left|U_{+, c}\right|^{2}-\left(\left|U_{H_{-, c}}\right|^{2}+\rho^{2}\left|U_{P_{-}}\right|^{2}\right) \\
& =C\left|U_{+, c}\right|^{2}+\left(\left|U_{H_{-,}}\right|^{2}+\rho^{2}\left|U_{P_{-}}\right|^{2}\right)-2\left(\left|U_{H_{-,}}\right|^{2}+\rho^{2}\left|U_{P_{-}}\right|^{2}\right) \\
& \geq C\left|U_{+, c}\right|^{2}+\left|U_{H_{-, c}}\right|^{2}+\rho^{2}\left|U_{P_{-}}\right|^{2}-C_{1}\left(\left|\Gamma_{1} U\right|^{2}+\left|U_{+, c}\right|^{2}\right) \\
& \geq C_{2}\left|U_{+, c}\right|^{2}+\left|U_{H_{-, c}}\right|^{2}+\rho^{2}\left|U_{P_{-}}\right|^{2}-C_{1}\left|\Gamma_{1} U\right|^{2} \\
& \geq C_{3}\left(\left|U_{+}\right|^{2}+\left|U_{H_{-}}\right|^{2}\right)+\rho^{2}\left|U_{P_{-}}\right|^{2}-C_{1}\left|\Gamma_{1} U\right|^{2}
\end{aligned}
$$

provided $C$ was big enough.

In addition we have

$$
\begin{gathered}
|(S F, U)| \leq\left|\left(S F_{P_{+}}, U_{P_{+}}\right)\right|+\left|\left(S F_{P_{-}}, U_{P_{-}}\right)\right|+\left|\left(S F_{H}, U_{H}\right)\right| \\
\leq\left(C_{\delta}\left|F_{P_{+}}\right|_{2}^{2}+\delta\left|U_{P_{+}}\right|_{2}^{2}\right)+\rho^{2}\left(C_{\delta}\left|F_{P_{-}}\right|_{2}^{2}+\delta\left|U_{P_{-}}\right|_{2}^{2}\right) \\
+\left(\frac{C_{\delta}}{\left(\gamma+\rho^{2}\right)}\left|F_{H}\right|_{2}^{2}+\delta\left(\gamma+\rho^{2}\right)\left|U_{H}\right|_{2}^{2}\right) .
\end{gathered}
$$

Plugging these estimates into the usual symmetrizer argument (recall (6.49)), we obtain after absorbing terms in the usual way the key small frequency estimate

$$
\begin{aligned}
& \left(\left|U_{P_{+}}\right|_{2}^{2}+\rho^{2}\left|U_{P_{-}}\right|_{2}^{2}+\left(\gamma+\rho^{2}\right)\left|U_{H}\right|_{2}^{2}\right) \\
& \quad+\left(\left|U_{+}\right|^{2}+\left|U_{H_{-}}\right|^{2}+\rho^{2}\left|U_{P_{-}}\right|^{2}\right) \\
& \leq C\left(\left|F_{P_{+}}\right|_{2}^{2}+\rho^{2}\left|F_{P_{-}}\right|_{2}^{2}+\frac{1}{\left(\gamma+\rho^{2}\right)}\left|F_{H}\right|_{2}^{2}\right)+C\left|\Gamma_{1} U\right|^{2}
\end{aligned}
$$

uniformly near $X_{0}$.

Assuming $\Gamma_{1} U=0$ as in (6.30), we deduce immediately from (8.11) our main estimate with $F$ as forcing

$$
|U|_{2}^{2} \leq C \frac{|F|_{2}^{2}}{\rho^{2}\left(\gamma+\rho^{2}\right)} .
$$

In particular with $\rho F$ forcing we obtain

$$
|U|_{2}^{2} \leq C \frac{|F|_{2}^{2}}{\left(\gamma+\rho^{2}\right)}
$$

In the mid-frequency region an argument identical to that for the nondegenerate problem (6.39) gives

$$
|U|_{2}^{2} \leq C|F|_{2}^{2}
$$

In this region the same estimate on traces as in (6.50) also holds. 
Remark 8.1. (1) Direct calculation using the explicit pointwise bounds on the resolvent kernel obtained in [Z1] reveals that bounds (8.11) and (8.14) are sharp.

(2) Using (7.31) in place of (7.30), we obtain instead of (8.9) the more precise estimate

$$
\left(\left(S+\Gamma_{1}^{*} \Gamma_{1}\right) U, U\right) \geq C\left(\left|U_{+}\right|^{2}+\left|U_{H_{-}}\right|^{2}+\left|U_{P_{2,-},}\right|^{2}\right)+\rho^{2}\left|U_{P_{1,-}}\right|^{2} .
$$

This refinement is put to important use in GMWZ1.

Remark 8.2. In view of the close relation between small-viscosity and low-frequency limits (see, e.g., discussion in Section 1.3 of [Z1] or Section 12 of [GMWZ1]), it is interesting to compare the small-frequency bounds (8.11) to the standard inviscid bounds of, e.g., [M1, Met2], which involve an additional variable $\varphi\left(x^{\prime}, t\right)$ recording shock location. Formally replacing this term $\varphi$ in the estimate for the inviscid problem with a viscous layer $\varphi\left(x^{\prime}, t\right) \psi^{\prime}(x) \sim \psi\left(x+\varphi\left(x^{\prime}, t\right)\right)-\psi(x)$, we obtain a slightly sharpened version of (8.11) which could have been obtained by segregating degenerate decaying parabolic modes in our analysis. However, we cannot make conclusions in the other direction by the present approach; that is, our bounds are consistent with but do not (quite) imply the inviscid ones. To recover the inviscid from viscous bounds would require the additional step of tracking viscous shock location (see [GMWZ2]).

8.1. Mixed norm estimate. Note that (8.13) implies both

$$
\begin{aligned}
& |U|_{2}^{2} \leq \frac{C}{\rho^{2}}|F|_{2}^{2} \text { and } \\
& \gamma|U|_{2}^{2} \leq C|F|_{2}^{2} .
\end{aligned}
$$

Let us work with the first now. Recall $|U|_{2}$ is the $L^{2}(x)$ norm, and define $V$ and $H$ by $U=\hat{V}(x, \tau, \gamma, \eta), F=\hat{H}, \gamma>0$. Suppose now that $d \geq 3$ and that $U$ and $F$ are supported in $\rho<\delta$.

(8.16) gives

$$
|U|_{2}^{2} \leq \frac{C}{|\tau, \eta|^{2}}|F|_{2}^{2}
$$

Integrate (8.17) $d \tau d \eta$ (dimension of $(\tau, \eta)$ space is $\geq 3$ ) to get

$$
\left|e^{-\gamma t} V\right|_{L^{2}(x, t, y)}^{2} \leq \int \frac{C}{|\tau, \eta|^{2}}|\hat{H}(x, \tau, \gamma, \eta)|_{L^{2}(x)}^{2} d \tau d \eta
$$

But

$$
|\hat{H}(x, \tau, \gamma, \eta)| \leq C|H(x, t, y)|_{L^{1}(t, y)},
$$

so

$$
\begin{aligned}
& |\hat{H}(x, \tau, \gamma, \eta)|_{L^{2}(x)}^{2} \leq C \int|H(x, t, y)|_{L^{1}(t, y)}^{2} d x \\
& \equiv|H|_{L^{2}\left(x, L^{1}(t, y)\right)}^{2} .
\end{aligned}
$$

Plug this into (8.18) to get

$$
\left|e^{-\gamma t} V\right|_{L^{2}(x, t, y)}^{2} \leq \int_{|\tau, \eta|<\delta} \frac{C}{|\tau, \eta|^{2}}|H|_{L^{2}\left(x, L^{1}(t, y)\right)}^{2} d \tau d \eta \leq C|H|_{L^{2}\left(x, L^{1}(t, y)\right)}^{2} .
$$

Let $\gamma \rightarrow 0$ to get

$$
|V|_{L^{2}(x, t, y)}^{2} \leq C|H|_{L^{2}\left(x, L^{1}(t, y)\right)}^{2} .
$$


8.2. Summary. Tracing back $U(\underline{8.1}) \rightarrow \mathcal{U}(\underline{2.19}) \rightarrow\left(\begin{array}{l}\hat{u} \\ \hat{v}\end{array}\right)(\underline{2.18})$, we have proved the following estimates for $L^{2}$ solutions $\hat{u}(x, \lambda, \eta)$ of

$$
\hat{u}_{x x}-(A(x) \hat{u})_{x}-s(x, \lambda, \eta) \hat{u}=\rho \hat{f}(x, \lambda, \eta) .
$$

Proposition 8.1. Let $\delta>0$ and $M>>\delta$.

(a) For $\hat{u}$ as in (8.23) and $\delta>0$ sufficiently small, we have

$$
|\hat{u}|_{2}^{2}+\left|\hat{u}_{x}\right|_{2}^{2} \leq C \frac{|\hat{f}|_{2}^{2}}{\left(\gamma+\rho^{2}\right)} \text { for } \rho=|\lambda, \eta| \leq \delta .
$$

(b) For $\delta \leq \rho \leq M$

$$
|\hat{u}|_{2}^{2}+\left|\hat{u}_{x}\right|_{2}^{2} \leq C|\hat{f}|_{2}^{2} .
$$

Observe that since $\rho$ is bounded in Proposition 8.1 we are free to multiply the left sides of the inequalities (8.24) and (8.25) by $\rho^{K}$ for any $K \geq 0$. Thus, estimate (8.22) and Proposition 8.1 imply the following proposition.

Let $\chi_{S}(\tau, \gamma, \eta), \chi_{M}$, and $\chi_{L}$ be smooth cutoffs supported respectively in $\rho \leq \delta$, $\delta / 2 \leq \rho \leq M$, and $M-1 \leq \rho$, such that

$$
\chi_{S}+\chi_{M}+\chi_{L}=1 .
$$

When we write $\chi(D) u$, we mean the function whose Fourier-Laplace transform is

$$
\chi(\tau, \gamma, \eta) \hat{u}(x, \tau, \gamma, \eta) .
$$

Here $\hat{u}$ is the Fourier transform of $e^{-\gamma t} \tilde{u}$, where $\tilde{u}$ is the extension of $u$ by 0 into $t<0$.

Proposition 8.2. (a) Suppose $d \geq 3$. For $u(x, t, y)$ as in (8.23) we have

$$
\left|\chi_{S}(D)\left(u, u_{y}\right)\right|_{L^{2}(x, t, y)}+\left|\chi_{S}(D) u_{x}\right|_{L^{2}(x, t, y)} \leq C|f|_{L^{2}\left(x, L^{1}(t, y)\right)} .
$$

(b) For $d \geq 1$

$$
\left|\chi_{M}(D)\left(u, u_{y}\right)\right|_{L^{2}(x, t, y)}+\left|\chi_{M}(D) u_{x}\right|_{L^{2}(x, t, y)} \leq C|f|_{L^{2}(x, t, y)} .
$$

\section{LARGE FREQUenCy ESTIMATE}

In the large frequency region, $\rho F$ forcing gives a worse estimate than $F$ forcing, and just plugging $\rho F$ into 6.50 is not helpful here (nor do the constants $C$ in the estimate remain uniformly bounded). Instead, one must take advantage of the fact that behavior for $\rho$ large is dominated by parabolic effects to obtain estimates by a different technique.

In [MZ1, this was carried out by a rescaling argument combined with appropriate symmetrizers, to which we could appeal here as well. Instead, we give an alternative argument similar to one in [KKP, based on direct integration by parts against the second order equation, which recovers the same results. We do not use a symmetrizer (more correctly, we take $S=I$ ); just pair with $\hat{u}(x, \lambda, \eta)$ and integrate by parts in the second order eigenvalue equation

$$
\hat{u}_{x x}-(A(x) \hat{u})_{x}-s(x, \lambda, \eta) \hat{u}=\hat{F}_{x}+i \eta \hat{G} .
$$


(Of course, the function $F$ in (9.1) is different from that in the previous section.) Here $\lambda=i \tau+\gamma$ and

$$
s(x, \lambda, \eta)=\sum_{j=2}^{d} A_{j}(x) i \eta_{j}+\lambda I+|\eta|^{2} I .
$$

Dropping hats, in the usual way one gets

$$
\left(\lambda+|\eta|^{2}\right)|u|_{2}^{2}+\left|u_{x}\right|_{2}^{2}=H\left(u, u_{x}, F, G\right)
$$

where

$$
|H| \leq C|u|_{2}\left|u_{x}\right|_{2}+|F|_{2}\left|u_{x}\right|_{2}+|G|_{2}|\eta||u|_{2}+C|\eta||u|_{2}^{2} .
$$

Take the modulus of each side of (9.2) (note $\gamma>0$ ), and absorb $\left|u_{x}\right|_{2}^{2}$ and $|\eta|^{2}|u|_{2}^{2}$ from the right to give, for some new constant,

$$
\left(|\tau|+\gamma+|\eta|^{2}\right)|u|_{2}^{2}+\left|u_{x}\right|_{2}^{2} \leq C\left(|u|_{2}^{2}+|F|_{2}^{2}+|G|_{2}^{2}\right) .
$$

By taking $\rho$ big and absorbing $C|u|_{2}^{2}$, we obtain

$$
\left(|\tau|+\gamma+|\eta|^{2}\right)|u|_{2}^{2}+\left|u_{x}\right|_{2}^{2} \leq C|F, G|_{2}^{2}, \text { for } \gamma>0 .
$$

Putting hats back and summarizing, we have shown that

$$
\left(|\tau|+\gamma+|\eta|^{2}\right)|\hat{u}|_{2}^{2}+\left|\hat{u}_{x}\right|_{2}^{2} \leq C|\hat{F}, \hat{G}|_{2}^{2}, \text { for } \gamma>0
$$

when $\hat{u}$ is supported in $\rho \geq M$ for $M$ sufficiently large. Thus, letting $\gamma \rightarrow 0$ and applying Parseval's formula, we get an estimate with no exponential weights

$$
\left|u, u_{y}, u_{t}\right|_{L^{2}(x, t, y)}+\left|u_{x}\right|_{L^{2}(x, t, y)} \leq C|F, G|_{L^{2}(x, t, y)} .
$$

Since cutoffs $\chi_{L}$ commute right through the eigenvalue equation (9.1), we have proved the following proposition.

Proposition 9.1. Suppose u satisfies

$$
\begin{aligned}
& \text { (a) } u_{t}+(A(x) u)_{x}+\sum_{j=2}^{d} A_{j}(x) \partial_{j} u-\triangle u=F_{x}+\operatorname{div}_{y} G, \\
& \text { (b) }\left.u\right|_{t=0}=0
\end{aligned}
$$

If $M$ is sufficiently large, then for $\chi_{L}$ supported in $\rho \geq M$,

$$
\left|\chi_{L}(D)\left(u, u_{y}, u_{t}\right)\right|_{L^{2}(x, t, y)}+\left|\chi_{L}(D) u_{x}\right|_{L^{2}(x, t, y)} \leq C|F, G|_{L^{2}(x, t, y)} .
$$

Here $u, F$, and $G$ in (9.8) (a) have been extended by zero into $t<0$ and, because of the initial condition $\left.u\right|_{t=0}=0$, the extensions satisfy (9.8) (a) for all $t$.

\section{LINEAR AND NONLINEAR STABILITY}

Proof of Theorem 4.1. The proof of this theorem will extend over the next few subsections. 
10.1. Strategy. Our first goal in this section is to prove the following estimate for solutions $u$ to (9.8) in space dimensions $d \geq 3$ :

$$
\left|u, u_{t}, u_{y}\right|_{L^{2}(x, t, y)}+\left|u_{x}\right|_{L^{2}(x, t, y)} \leq C\left(|F, G|_{L^{2}\left(x, L^{1}(t, y)\right)}+|F, G|_{L^{2}(x, t, y)}\right) .
$$

Clearly, it remains to treat only $\chi_{S}(D) u$ and $\chi_{M}(D) u$. Accordingly, for the rest of this discussion, we restrict attention to $|(\tau, \gamma, \eta)|$ uniformly bounded.

As in [KK] let $\hat{u}=\hat{w}+\hat{u}_{1}$, where $\hat{w}(x, \lambda, \eta)$ satisfies

$$
\hat{w}_{x x}-(A(x) \hat{w})_{x}=\hat{F}_{x}, \quad|\hat{w}|_{2}<\infty .
$$

This piece integrates to

$$
\hat{w}_{x}-A(x) w=\hat{F}(x, \lambda, \eta) .
$$

This is the auxiliary problem for which we obtain a solution satisfying the following $L^{2}$ :

$$
|\hat{w}|_{L^{2}(x)} \leq C|\hat{F}|_{L^{2}(x)} .
$$

Next, $\hat{u}_{1}=\hat{u}-\hat{w}$ satisfies

$$
\hat{u}_{1 x x}-\left(A(x) \hat{u}_{1}\right)_{x}-s(x, \lambda, \eta) \hat{u}_{1}=s \hat{w}+i \eta \cdot \hat{G}, \quad\left|\hat{u}_{1}\right|_{2}<\infty .
$$

The right side of 10.5 qualifies as " $\rho F$ " forcing, so we may apply Proposition 8.1 and the estimate (10.4) to establish

$$
|\hat{u}|_{L^{2}(x)} \leq C\left(\left|\hat{u}_{1}\right|_{L^{2}(x)}+|\hat{w}|_{L^{2}(x)}\right) \leq \frac{C\left(|\hat{F}|_{L^{2}(x)}+|\hat{G}|_{L^{2}(x)}\right)}{\left(\gamma+\rho^{2}\right)} .
$$

By the calculation (8.21) 8.22 , this yields

Proposition 10.1. (a) Suppose $d \geq 3$. For $u_{1}(x, t, y)$ as in (10.5) we have

$$
\left|\chi_{S}(D)\left(u_{1}, u_{1 y}\right)\right|_{L^{2}(x, t, y)}+\left|\chi_{S}(D) u_{1 x}\right|_{L^{2}(x, t, y)} \leq C|F, G|_{L^{2}\left(x, L^{1}(t, y)\right)} .
$$

(b) For $d \geq 1$

$$
\left|\chi_{M}(D)\left(u_{1}, u_{1 y}\right)\right|_{L^{2}(x, t, y)}+\left|\chi_{M}(D) u_{1 x}\right|_{L^{2}(x, t, y)} \leq C|F, G|_{L^{2}(x, t, y)} .
$$

To complete the proof of (10.1), we just need to show (10.4).

10.2. Auxiliary problem. In the problem (10.3) the matrix $A(x)$ is independent of frequency. To prove the $L^{2}$ estimate (10.4), we consider the $n \times n$ system on $\mathbb{R}$

$$
w_{x}-A(x) w=f(x),
$$

and we show that there is a solution satisfying

$$
|w|_{L^{2}(x)} \leq C|f|_{L^{2}(x)} .
$$

This implies there is a solution to 10.3 satisfying

$$
|\hat{w}(x, \lambda, \eta)|_{L^{2}(x)} \leq C|\hat{F}(x, \lambda, \eta)|_{L^{2}(x)},
$$

with $C$ independent of frequency, which will give (10.4).

Consider the doubled $2 n \times 2 n$ boundary problem on $x \geq 0$ equivalent to (10.9)

$$
\begin{aligned}
& \text { (a) } \quad W_{x}-\mathcal{A}(x) W=\mathcal{F}(x), \\
& \text { (b) } B W=0 \text { on } x=0,
\end{aligned}
$$


where

$$
\begin{aligned}
& W(x)=\left(w_{+}(x), w_{-}(x)\right), \\
& \mathcal{A}(x)=\left(\begin{array}{cc}
A_{+}(x) & 0 \\
0 & -A_{-}(x)
\end{array}\right), \\
& \mathcal{F}(x)=\left(f_{+}(x),-f_{-}(x)\right), \text { and } \\
& B W=w_{+}-w_{-} .
\end{aligned}
$$

Let $\mathcal{E}_{-}(0)$ be the space of boundary values of decaying solutions of 10.12 (a) when $\mathcal{F}=0$. Hypothesis (H3) together with classical ODE results ([Co]) implies

$$
\operatorname{dim} \mathcal{E}_{-}(0)=(n-k)+(n-l)=n+1 .
$$

On the other hand $\operatorname{ker} B$ has dimension $n$. A basis for it is

$$
\left\{E_{1}, \ldots, E_{n}\right\} \text {, where } E_{j}=\left(e_{j}, e_{j}\right),
$$

and the $e_{j}$ are the standard basis vectors of $\mathbb{C}^{n}$.

Hypothesis (H4) implies $\operatorname{ker} B$ and $\mathcal{E}_{-}(0)$ have a one-dimensional intersection spanned by

$$
P=(\phi(0), \phi(0))
$$

We now define an augmented boundary condition $\tilde{B}$ with the property that

$$
\mathbb{C}^{2 n}=\operatorname{ker} \tilde{B} \oplus \mathcal{E}_{-}(0) .
$$

Some component of $\phi(0)$, say the first, is not zero. Let $W_{1}$ denote the first component of $W$. Then we may simply set

$$
\tilde{B} W=\left(W_{1}, w_{+}-w_{-}\right) \in \mathbb{C}^{n+1},
$$

so that (10.17) holds.

Now we can estimate solutions to

$$
\begin{aligned}
& W_{x}-\mathcal{A}(x) W=\mathcal{F}(x), \\
& \tilde{B} W=0 \text { on } x=0,
\end{aligned}
$$

using an idea of [MZ1. Note that any solution of (10.19) is also a solution of (10.12).

Construct as in [MZ1] a conjugator $\mathcal{C}(x)$ on $x \geq 0$ satisfying

$$
\mathcal{C}^{-1} \text { is uniformly bounded, }
$$

$$
\begin{aligned}
& \mathcal{C}(x)=I d+O\left(e^{-\beta x}\right) \text { for some } \beta>0, \\
& \mathcal{C}_{x}(x)=\mathcal{A}(x) \mathcal{C}(x)-\mathcal{C}(x) \mathcal{A}(\infty)
\end{aligned}
$$

Setting $W=\mathcal{C} V$ transforms 10.19 into

$$
\begin{aligned}
& V_{x}-\mathcal{A}(\infty) V=\mathcal{H}, \\
& \tilde{\mathcal{B}} V=0 \text { on } x=0,
\end{aligned}
$$

where $\mathcal{H}=\mathcal{C}^{-1} \mathcal{F}$ and $\tilde{\mathcal{B}}=\tilde{B} \mathcal{C}$. The properties of $\mathcal{C}$ imply immediately that an estimate for (10.21) gives an estimate for (10.19).

Since $\mathcal{C}$ preserves the decomposition (10.17), it is now an easy matter to construct a symmetrizer for (10.21) and to obtain

$$
|V|_{L^{2}(x)} \leq C|\mathcal{H}|_{L^{2}(x)} .
$$


Thus, $W=\mathcal{C} V$ satisfies

$$
|W|_{L^{2}(x)} \leq C|\mathcal{F}|_{L^{2}(x)}
$$

and estimate (10.10) follows.

Remark 10.1. The improved bounds available for divergence forcing are connected with the fact [Z1] that the only quantities conserved by the linearized equations are those afforded by conservation of mass, a property which holds also for overcompressive but not for undercompressive shocks. Recall, in the notation of (2.2), with $k+\ell=n+r, r \geq 2$ corresponds to the overcompressive case, $r=1$ to the Lax case, and $r \leq 0$ to the undercompressive case. Likewise, it is readily seen that the auxiliary equation construction of $[\mathrm{KK}]$ goes through essentially unchanged for the overcompressive case, where the augmented boundary condition $\tilde{B}$ again has $r$ extra constraints (now $>1$ ), to yield the same bounds as in the Lax case. Thus, we may obtain as in [Z1] the same nonlinear stability results in the overcompressive case as in the Lax case. The auxiliary equation construction fails in the undercompressive case, however; indeed, this case is essentially different, as discussed further in [Z1], [Z2].

\subsection{Linear stability.}

Notation 10.1. (1) $|v(t, y)|_{L_{T}^{2}(t, y)}$ is the $L^{2}$ norm on $[0, T] \times \mathbb{R}_{y}^{n-1}$.

(2) $|u|_{2 ; p}^{2}=\sum_{|\alpha| \leq p}\left|\partial_{(x, y)}^{\alpha} u(x, t, y)\right|_{L^{2}(x, t, y)}^{2}$.

(3) $|u|_{(2,1) ; p}^{2}=\sum_{|\alpha| \leq p}\left|\partial_{(x, y)}^{\alpha} u(x, t, y)\right|_{L^{2}\left(x, L^{1}(t, y)\right)}^{2}$

(4) $|u|_{2 ; p, T}^{2}=\sum_{|\alpha| \leq p}\left|\partial_{(x, y)}^{\alpha} u(x, t, y)\right|_{L^{2}\left(x, L_{T}^{2}(t, y)\right)}^{2}$.

(5) $|u|_{(2,1) ; p, T}^{2}=\sum_{|\alpha| \leq p}\left|\partial_{(x, y)}^{\alpha} u(x, t, y)\right|_{L^{2}\left(x, L_{T}^{1}(t, y)\right)}^{2}$.

(6) $|u|_{(\infty, 2) ; p, T}=\sum_{|\alpha| \leq p}\left|\partial_{(x, y)}^{\alpha} u(x, t, y)\right|_{L^{\infty}\left(x, L_{T}^{2}(t, y)\right)}$.

The proof of nonlinear stability depends on the following estimates for solutions of

$$
\begin{aligned}
& \text { (a) } u_{t}+(A(x) u)_{x}+\sum_{j=2}^{d} A_{j}(x) \partial_{j} u-\triangle u=F_{x}+\operatorname{div}_{y} G, \\
& \text { (b) }\left.u\right|_{t=0}=0 .
\end{aligned}
$$

Proposition 10.2 (Main linear estimate).

$$
\begin{aligned}
& \text { (a) }|u|_{2 ; 1}+\left|u_{t}\right|_{2 ; 0} \leq C\left(|F, G|_{(2,1) ; 0}+|F, G|_{2 ; 0}\right) . \\
& \text { (b) }|u|_{2 ; p+1}+\left|u_{t}\right|_{2 ; p} \leq C_{p}\left(|F, G|_{(2,1) ; 0}+|F, G|_{2 ; p}\right) . \\
& \text { (c) }|u|_{2 ; p+1, T}+\left|u_{t}\right|_{2 ; p, T} \leq C_{p}\left(|F, G|_{(2,1) ; 0, T}+|F, G|_{2 ; p, T}\right) .
\end{aligned}
$$

A key point is that $C$ is independent of $T$ and there are no exponential weights in the norms.

Proof. Estimate (a) is (10.1), whose proof has just been completed.

(c) follows from (b) and the fact that the future does not affect the past.

Care is needed in the proof of (b) because our basic estimate is asymmetric; there is a mixed norm on the right but not the left (the argument of [KK] is incomplete here but can be fixed by an argument like the following one). Note that if one just differentiates (10.24) with respect to $x$ and applies (10.25) (a), there is no way to absorb the mixed norm of terms like $\left(A^{\prime}(x) u\right)_{x}$ that get thrown on the right as forcing. 
Recall that $u, F$, and $G$ in (10.24) (a) have been extended by zero into $t<0$ and, because of the initial condition $\left.u\right|_{t=0}=0$, the extensions satisfy (10.24) (a) for all $t$.

1. Let $\chi(\tau, \eta)$ be a smooth cutoff function supported in $|\tau, \eta| \leq C$ for $C$ to be chosen sufficiently large. Since $\chi$ has bounded support in the frequency variables,

$$
\left|\chi(D) \partial_{t}^{k} \partial_{y}^{\alpha}\left(u, u_{x}\right)\right|_{2 ; 0} \leq C\left|u, u_{x}\right|_{2 ; 0},
$$

so we immediately obtain from (10.25) (a)

$$
\left|\chi(D) \partial_{t}^{k} \partial_{y}^{\alpha}\left(u, u_{x}\right)\right|_{2 ; 0} \leq C\left(|F, G|_{(2,1) ; 0}+|F, G|_{2 ; 0}\right) .
$$

2. Note that $\chi(D)$ commutes right through (10.24) (a) and that

$$
\begin{aligned}
& |\chi(D) f(x, t, y)|_{(2,1) ; 0} \leq C|f|_{(2,1) ; 0}, \text { since } \\
& |\chi(D) g(t, y)|_{L^{1}(t, y)} \leq C|g(t, y)|_{L^{1}(t, y)} .
\end{aligned}
$$

The latter inequality is easily seen by writing $\chi(D) g$ as a convolution.

Thus, after solving for $\chi(D) u_{x x}$ using the equation (10.24) (a), we can use the estimates (10.27) and (10.28) to get

$$
\left|\chi(D) \partial_{y}^{\alpha} u_{x x}\right|_{2 ; 0} \leq C\left(|F, G|_{(2,1) ; 0}+|F, G|_{2 ; 1}\right) .
$$

3. Differentiating the equation $(10.24)$ (a) with respect to $x$ and using the estimates (10.27) and (10.29) give for $|\alpha|+l \leq p+1$

$$
\left|\chi(D) \partial_{y}^{\alpha} \partial_{x}^{l} u\right|_{2 ; 0} \leq C\left(|F, G|_{(2,1) ; 0}+|F, G|_{2 ; p}\right)
$$

Here one estimates terms involving $x$ derivatives of order $k$ by using the equation to express them as sums of terms involving $x$ derivatives of order $\leq k-1$ which have already been estimated.

As before we can insert $\partial_{t}^{k}$ in the left sides of (10.29) and (10.30).

4. Next consider the region where $|\tau, \eta|$ is big. Here we need a different argument. In this region we have the pure $L^{2}$ estimates (9.6) and (9.7) with no mixed norms. We can differentiate 10.24) (a) with respect to $x$ or $y$ and apply Proposition 9.1 to the differentiated problem, after observing that conservative forcing is maintained. For example, differentiate (10.24) (a) once with respect to $x$ to get

$$
\begin{aligned}
\left(u_{x}\right)_{t} & +\left(A(x) u_{x}\right)_{x}+\sum_{j=2}^{d} A_{j}(x) \partial_{j} u_{x}-\triangle u_{x} \\
& =F_{x x}+\operatorname{div}_{y} G_{x}-\left(\left(A^{\prime}(x) u\right)_{x}+\sum_{j=2}^{d}\left(A_{j}^{\prime}(x) u\right)_{y_{j}}\right) .
\end{aligned}
$$

Apply Proposition 9.1 to obtain

$$
\left|(1-\chi(D)) u_{x x}\right|_{2 ; 0} \leq C|F, G|_{2 ; 1} .
$$

Continuing in this way, we obtain for $|\alpha|+l \leq p+1$

$$
\left|(1-\chi(D)) \partial_{y}^{\alpha} \partial_{x}^{l} u\right|_{2 ; 0} \leq C|F, G|_{2 ; p} .
$$

5. In the same way we obtain for $|\alpha|+l \leq p$

$$
\left|(1-\chi(D)) \partial_{y}^{\alpha} \partial_{x}^{l} u_{t}\right|_{2 ; 0} \leq C|F, G|_{2 ; p} .
$$

6. Use (10.30), 10.33), and (10.34) to get for $|\alpha|+l \leq p+1$

$$
\left|\partial_{y}^{\alpha} \partial_{x}^{l} u\right|_{2 ; 0} \leq C\left(|F, G|_{(2,1) ; 0}+|F, G|_{2 ; p}\right)
$$


and for $|\alpha|+l \leq p$

$$
\left|\partial_{y}^{\alpha} \partial_{x}^{l} u_{t}\right|_{2 ; 0} \leq C\left(|F, G|_{(2,1) ; 0}+|F, G|_{2 ; p}\right) .
$$

This gives (b).

10.4. Nonlinear long time stability. First, let us rewrite the error equation (2.14) in the $(x, t, y)$ notation as

$$
\begin{aligned}
& u_{t}+(A(x) u)_{x}+\sum_{j=2}^{d} A_{j}(x) \partial_{y_{j}} u+\epsilon \operatorname{div}(B(x, t, y) u)+\epsilon \operatorname{div}(h(x, t, y, u)) \\
& =\triangle u+\operatorname{div}((F, G)(x, t, y)) \\
& \left.u\right|_{t=0}=0 .
\end{aligned}
$$

In order to complete the proof of Theorem 4.1 we need to show that for $\epsilon$ small enough, the solution to (10.37) exists for all time and satisfies

$$
|u(x, t, y)|_{L^{\infty}(x, y)} \rightarrow 0 \text { as } t \rightarrow \infty .
$$

Local existence in time is well known. We use an argument similar to the one in [KK], except that we have the mixed norm in place of their $L^{1}$ norm.

For $p>\frac{d}{2}$ ( $d$ is the number of space dimensions) set

$$
E_{p}=C_{p}\left(|F, G|_{(2,1) ; 0}+|F, G|_{2 ; p}\right) .
$$

Assumption $(\mathrm{H} 5)^{I}$ on $v_{0}$ implies $E_{p}<\infty$.

For $T_{\epsilon}$ sufficiently small the solution of (10.37) satisfies

$$
|u|_{2 ; p+1, T_{\epsilon}}+\left|u_{t}\right|_{2 ; p, T_{\epsilon}} \leq E_{p}+1 .
$$

In fact, let us suppose now that $T_{\epsilon}$ in (10.40) is the maximal $T_{\epsilon}$ for which (10.40) holds. We will show that for $\epsilon$ small enough

$$
T_{\epsilon}=\infty \text {. }
$$

In turn this implies (10.38).

We now consider $\epsilon \operatorname{div}(B(x, t, y) u)+\epsilon \operatorname{div}(h(x, t, y, u))$ as part of the forcing, and apply the main linear estimate to obtain

$$
\begin{aligned}
& |u|_{2 ; p+1, T_{\epsilon}}+\left|u_{t}\right|_{2 ; p, T_{\epsilon}} \\
& \quad \leq C_{p}\left(|(F, G)+\epsilon B u+\epsilon h|_{(2,1) ; 0, T_{\epsilon}}+|(F, G)+\epsilon B u+\epsilon h|_{2 ; p, T_{\epsilon}}\right) \\
& \quad \leq E_{p}+C \epsilon\left(|B u|_{(2,1) ; 0, T_{\epsilon}}+|B u|_{2 ; p, T_{\epsilon}}+|h|_{(2,1) ; 0, T_{\epsilon}}+|h|_{2 ; p, T_{\epsilon}}\right) .
\end{aligned}
$$

Since we have local existence, all we need to do is show that each of the four terms

$$
|B u|_{(2,1) ; 0, T_{\epsilon}},|B u|_{2 ; p, T_{\epsilon}},|h|_{(2,1) ; 0, T_{\epsilon}},|h|_{2 ; p, T_{\epsilon}}
$$

is bounded by some function $f\left(E_{p}\right)$.

Notation 10.2. (a) For $k \in\{1,2,3, \ldots\}$ let $\partial^{k}$ denote the collection of operators $\partial_{(x, y)}^{\alpha}$ with $|\alpha|=k$ ( $\alpha$ is a multi-index). Sometimes $\partial^{k}$ is used to denote a particular member of this collection. Set $\partial^{0} \phi=\phi$.

(b) For $k \in\{1,2,3, \ldots\}$ denote by $\partial^{\langle k\rangle} \phi$ the set of products of the form $\left(\partial^{\alpha_{1}} \phi_{i_{1}}\right) \cdots\left(\partial^{\alpha_{r}} \phi_{i_{r}}\right)$ where $1 \leq r \leq k, \alpha_{1}+\cdots+\alpha_{r}=k, \alpha_{i} \geq 1$. Set $\partial^{\langle 0\rangle} \phi=1$.

(c) Set $|v|_{\infty, T}=|v|_{L^{\infty}\left([0, T] \times \mathbb{R}^{d}\right)},|v|_{\infty}=|v|_{L^{\infty}\left([0, \infty] \times \mathbb{R}^{d}\right)}$. 
(d) $\Phi=\Phi(x, t, y, u)$ will always denote a smooth function of its arguments with the property that $|\Phi| \leq C_{M}$ when $|u| \leq M$. It may change from line to line.

Lemma 10.1 (Moser inequality). For $p \in\{0,1,2,3, \ldots\}$ let $\alpha_{1}+\cdots+\alpha_{r} \leq p, \alpha_{i} \in$ $\{0,1,2,3, \ldots\}$. Suppose $\left|v_{i}\right|_{2 ; p, T}+\left|v_{i}\right|_{\infty, T}<\infty$. Then there exists $C$ independent of $T$ such that

$$
\left|\left(\partial^{\alpha_{1}} v_{1}\right) \cdots\left(\partial^{\alpha_{r}} v_{r}\right)\right|_{2 ; 0, T} \leq C \sum_{i=1}^{r}\left|v_{i}\right|_{2 ; p, T}\left(\prod_{j \neq i}\left|v_{i}\right|_{\infty, T}\right) .
$$

\section{Lemma 10.2.}

$$
|u v|_{(2,1) ; 0, T}^{2} \leq|u|_{(\infty, 2) ; 0, T}^{2}|v|_{2 ; 0, T}^{2} .
$$

Proof. Write down the definition of the left side, apply Cauchy-Schwartz in $(t, y)$, and pull out $|u|_{(\infty, 2) ; 0, T}^{2}$ from the $d x$ integral.

As a final preliminary step, note that assumption (H5) implies

$$
\left|e^{-t} v_{0}(x, y)\right|_{2 ; p}<\infty
$$

so by (10.40), 10.45), and Sobolev inequalities we have

$$
\begin{aligned}
& \text { (a) }|u|_{L^{\infty}\left(\left[0, T_{\epsilon}\right] \times \mathbb{R}^{d}\right)} \leq f\left(E_{p}\right), \\
& \text { (b) }|u|_{(\infty, 2) ; p, T_{\epsilon}} \leq f\left(E_{p}\right),
\end{aligned}
$$$$
\text { (c) }\left|e^{-t} v_{0}\right|_{\infty}<\infty \text {. }
$$

First we show

$$
|h|_{2 ; p, T_{\epsilon}} \leq f\left(E_{p}\right) .
$$

Let $k \leq p$. Recall using (2.10) that $h(x, t, y, u)=H\left(\psi, e^{-t} v_{0}, u\right) u^{2}$ (obvious notation), so $\left|\partial^{k} h\right|_{2 ; 0, T_{\epsilon}}$ is a sum of terms of the form

$$
\left|\Phi \partial^{\langle j\rangle}\left(e^{-t} v_{0}\right) \partial^{\langle l\rangle} u \partial^{m} u \partial^{n} u\right|_{2 ; 0, T_{\epsilon}},
$$

where $l+m+n \leq k$. Use the Moser inequality (with (10.40), (10.45), (10.46) ) to see that $(10.48) \leq f\left(E_{p}\right)$.

This gives (10.47). The treatment of $|B u|_{2 ; p, T_{\epsilon}}$ is similar.

Next we show

$$
|h|_{(2,1) ; 0, T_{\epsilon}} \leq f\left(E_{p}\right) .
$$

Using the same expression for $h$ as before and applying Lemma 10.2 we have

$$
|h|_{(2,1) ; 0, T_{\epsilon}}=\left|H u^{2}\right|_{(2,1) ; 0, T_{\epsilon}} \leq|u|_{(\infty, 2) ; 0, T_{\epsilon}}|H u|_{2 ; 0, T_{\epsilon}} \leq f\left(E_{p}\right),
$$

where the last inequality follows from (10.40) and (10.46). This proves (10.49).

The term $|B u|_{(2,1) ; 0, T_{\epsilon}}$ is, again, similar, so this completes the proof that for $\epsilon$ small enough, $T_{\epsilon}=\infty$. Thus, the proof of Theorem 4.1 is finished.

Proof of Theorem 4.2. Consider again the linear problem (9.8) with divergence form forcing.

1. Linear estimates. In Section 12 (see Corollary 12.1 below) we prove by a different argument that uses (H6) the following estimate for solutions $u$ to (9.8) for all dimensions $d \geq 1$ :

$$
\left|u, u_{y}, u_{t}\right|_{2 ; 0}+\left|u_{x}\right|_{2 ; 0} \leq C\left(|F, G|_{1 ; 0}+|F, G|_{2 ; 0}\right) .
$$


As before, repetition of the proof of Proposition 10.2 gives

$$
|u|_{2 ; p+1, T}+\left|u_{t}\right|_{2 ; p, T} \leq C_{p}\left(|F, G|_{1 ; 0, T}+|F, G|_{2 ; p, T}\right) .
$$

2. Nonlinear estimates. For $p>\frac{d}{2}$ and $F, G$ as in (10.37) set

$$
E_{p}=C_{p}\left(|F, G|_{1 ; 0}+|F, G|_{2 ; p}\right)
$$

and observe that assumption $(\mathrm{H} 5)^{I V}$ on $v_{0}$ implies $E_{p}<\infty$.

For $T_{\epsilon}$ sufficiently small the solution of (10.37) satisfies

$$
|u|_{2 ; p+1, T_{\epsilon}}+\left|u_{t}\right|_{2 ; p, T_{\epsilon}} \leq E_{p}+1 .
$$

Now we have

$$
\begin{aligned}
& |u|_{2 ; p+1, T_{\epsilon}}+\left|u_{t}\right|_{2 ; p, T_{\epsilon}} \\
& \quad \leq E_{p}+C \epsilon\left(|B u|_{1 ; 0, T_{\epsilon}}+|B u|_{2 ; p, T_{\epsilon}}+|h|_{1 ; 0, T_{\epsilon}}+|h|_{2 ; p, T_{\epsilon}}\right) .
\end{aligned}
$$

Consider $|h|_{1 ; 0, T_{\epsilon}}$ for $h=H u^{2}$ (earlier notation). We have by Cauchy-Schwartz

$$
\left|H u^{2}\right|_{1 ; 0, T_{\epsilon}} \leq|H|_{\infty, T_{\epsilon}}|u|_{2 ; 0, T_{\epsilon}}^{2} \leq f\left(E_{p}\right) .
$$

$|B u|_{1 ; 0, T_{\epsilon}}$ is similar and the remaining terms are just as in the proof of Theorem 4.1, so the proof of Theorem 4.2 is finished.

\section{Part 4. Nonzero mass perturbations}

\section{Nonlinear STABILITy}

In this section we prove Theorem 4.3 using the linear estimates from earlier sections. We also prove Theorem 4.4 assuming the $L^{1}-L^{2}$ estimates proved in the next section. The passage from linear to nonlinear stability in both cases is very similar to the argument in Section 9.

Proof of Theorem 4.3.

1. Error equation. We no longer have a perturbation in conservative form, so we must work with the error problem

$$
\begin{aligned}
& \begin{aligned}
u_{t}+(A(x) u)_{x} & +\sum_{j=2}^{d} A_{j}(x) \partial_{j} u+\epsilon \operatorname{div}(B(x, t, y) u)+\epsilon \operatorname{div}(h(x, t, y, u)) \\
& =\triangle u+f
\end{aligned} \\
& \begin{array}{ll}
\left.u\right|_{t=0}=0, &
\end{array}
\end{aligned}
$$

(this is (2.12) in $(x, t, y)$ notation) instead of 2.14). Here $f$ is the particular function appearing in (2.12). Consider also the corresponding linear problem

$$
\begin{aligned}
& u_{t}+(A(x) u)_{x}+\sum_{j=2}^{d} A_{j}(x) \partial_{y_{j}} u-\triangle u=f, \\
& \left.u\right|_{t=0}=0
\end{aligned}
$$

for any $f \in L^{2}\left([0, \infty) \times \mathbb{R}^{d}\right)$. As usual, $u$ and $f$ are extended by zero in $t<0$.

2. Linear estimates. Recall the small frequency estimate (8.12) for general forcing

$$
|U|_{2}^{2} \leq C \frac{|F|_{2}^{2}}{\rho^{2}\left(\gamma+\rho^{2}\right)} \leq C \frac{|F|_{2}^{2}}{\rho^{4}}
$$


where $U(x, \lambda, \eta), F(x, \lambda, \eta)$ are related to $u(x, t, y), f(x, t, y)$ just as they were in the doubled boundary problem (8.1). (Recall that in Section 7 the assumption of " $\rho F$ " forcing is not invoked until (8.13).) Since $d \geq 5$ now, the argument used to obtain the earlier mixed norm estimate gives

$$
|V|_{L^{2}(x, t, y)} \leq C|H|_{L^{2}\left(x, L^{1}(t, y)\right)},
$$

where $U=\hat{V}(x, \lambda, \eta), F=\hat{H}$. There is no need to consider an auxiliary problem. For $u$ as in (11.2) this gives

$$
\begin{array}{r}
\left|\chi_{S}(D)\left(u, u_{y}\right)\right|_{L^{2}(x, t, y)}+\left|\chi_{S}(D) u_{x}\right|_{L^{2}(x, t, y)} \leq C|f|_{L^{2}\left(x, L^{1}(t, y)\right)}, \\
\left|\chi_{M}(D)\left(u, u_{y}\right)\right|_{L^{2}(x, t, y)}+\left|\chi_{M}(D) u_{x}\right|_{L^{2}(x, t, y)} \leq C|f|_{L^{2}(x, t, y)}
\end{array}
$$

where the medium frequency estimate is proved just as before.

For the large frequency estimate take the $L^{2}(x)$ pairing of $\hat{u}(x, \lambda, \eta)$ with the eigenvalue equation

$$
\hat{u}_{x x}-(A(x) \hat{u})_{x}-s(x, \lambda, \eta) \hat{u}=\hat{f}(x, \lambda, \eta)
$$

and argue as in Section 9 to obtain

$$
\left|\chi_{L}(D)\left(u, u_{y}, u_{t}\right)\right|_{L^{2}(x, t, y)}+\left|\chi_{L}(D) u_{x}\right|_{L^{2}(x, t, y)} \leq C|f|_{L^{2}(x, t, y)} .
$$

Adding up, for solutions of (11.2) we have

$$
\left|u, u_{y}, u_{t}\right|_{2 ; 0}+\left|u_{x}\right|_{2 ; 0} \leq C\left(|f|_{(2,1) ; 0}+|f|_{2 ; 0}\right) .
$$

Line by line repetition of the proof of Proposition 10.2 gives the higher derivative estimates:

$$
|u|_{2 ; p+1, T}+\left|u_{t}\right|_{2 ; p, T} \leq C_{p}\left(|f|_{(2,1) ; 0, T}+|f|_{2 ; p, T}\right) .
$$

Note that there is a gain of one derivative in this estimate, while in the estimate of Proposition 10.2 there is a gain of two derivatives since the forcing there is $\operatorname{div}(F, G)$.

3. Nonlinear estimates. We will refer to the corresponding arguments in Section 9 , just indicating the needed changes.

For $p>\frac{d}{2}$ and $f$ as in (11.1) set

$$
E_{p}=C_{p}\left(|f|_{(2,1) ; 0}+|f|_{2 ; p}\right) .
$$

Assumption (H5) ${ }^{I I}$ on $v_{0}$ implies $E_{p}<\infty$.

For $T_{\epsilon}$ sufficiently small the solution of (11.1) satisfies

$$
|u|_{2 ; p+1, T_{\epsilon}}+\left|u_{t}\right|_{2 ; p, T_{\epsilon}} \leq E_{p}+1 .
$$

In place of (10.42) for solutions to (11.1) we now have

$$
\begin{aligned}
& |u|_{2 ; p+1, T_{\epsilon}}+\left|u_{t}\right|_{2 ; p, T_{\epsilon}} \\
& \quad \leq C_{p}\left(|f+\epsilon \operatorname{div}(B u)+\epsilon \operatorname{div}(h)|_{(2,1) ; 0, T_{\epsilon}}+|f+\epsilon \operatorname{div}(B u)+\epsilon \operatorname{div}(h)|_{2 ; p, T_{\epsilon}}\right) \\
& \quad \leq E_{p}+C \epsilon\left(|B u|_{(2,1) ; 1, T_{\epsilon}}+|B u|_{2 ; p+1, T_{\epsilon}}+|h|_{(2,1) ; 1, T_{\epsilon}}+|h|_{2 ; p+1, T_{\epsilon}}\right) .
\end{aligned}
$$

Just as before, the Moser inequalities imply

$$
|B u|_{2 ; p+1, T_{\epsilon}}+|h|_{2 ; p+1, T_{\epsilon}} \leq f\left(E_{p}\right) .
$$

Also, $|h|_{(2,1) ; 1, T_{\epsilon}}$ is a sum of terms of the form

$$
\left|\Phi \partial^{\langle j\rangle}\left(e^{-t} v_{0}\right) \partial^{\langle l\rangle} u \partial^{m} u \partial^{n} u\right|_{(2,1) ; 0, T_{\epsilon}},
$$


where $j+l+m+n \leq 1$. By Lemma 10.2, (11.14) $\leq$

$$
C\left|\partial^{m} u\right|_{(\infty, 2) ; 0, T_{\epsilon}}\left|\partial^{\langle j\rangle}\left(e^{-t} v_{0}\right) \partial^{\langle l\rangle} u \partial^{n} u\right|_{2 ; 0, T_{\epsilon}},
$$

and the second and third factors are $\leq f\left(E_{p}\right)$ by (10.45) and the Moser inequality, respectively. 4.3 .

The term $|B u|_{(2,1) ; 1, T_{\epsilon}}$ is treated similarly, so this concludes the proof of Theorem

Notation 11.1. (1) $|u|_{1 ; p}=\sum_{|\alpha| \leq p}\left|\partial_{(x, y)}^{\alpha} u(x, t, y)\right|_{L^{1}(x, t, y)}$.

(2) $|u|_{1 ; p, T}=\sum_{|\alpha| \leq p}\left|\partial_{(x, y)}^{\alpha} u(x, t, y)\right|_{L^{1}\left(x, L_{T}^{1}(t, y)\right)}$.

Proof of Theorem 4.4. Again, consider the error equation (11.1) and the corresponding linear problem (11.2).

1. Linear estimates. In the next section (see Corollary 12.1 below) we prove the following estimate for solutions $u$ to (11.2):

$$
\left|u, u_{y}, u_{t}\right|_{2 ; 0}+\left|u_{x}\right|_{2 ; 0} \leq C\left(|f|_{1 ; 0}+|f|_{2 ; 0}\right) .
$$

As before, repetition of the proof of Proposition 10.2 gives

$$
|u|_{2 ; p+1, T}+\left|u_{t}\right|_{2 ; p, T} \leq C_{p}\left(|f|_{1 ; 0, T}+|f|_{2 ; p, T}\right) .
$$

2. Nonlinear estimates. For $p>\frac{d}{2}$ and $f$ as in (11.1) set

$$
E_{p}=C_{p}\left(|f|_{1 ; 0}+|f|_{2 ; p}\right)
$$

and observe that assumption (H5) ${ }^{I I I}$ on $v_{0}$ implies $E_{p}<\infty$.

For $T_{\epsilon}$ sufficiently small the solution of (11.1) satisfies

$$
|u|_{2 ; p+1, T_{\epsilon}}+\left|u_{t}\right|_{2 ; p, T_{\epsilon}} \leq E_{p}+1 .
$$

Now we have

$$
\begin{aligned}
& |u|_{2 ; p+1, T_{\epsilon}}+\left|u_{t}\right|_{2 ; p, T_{\epsilon}} \\
& \quad \leq E_{p}+C \epsilon\left(|B u|_{1 ; 1, T_{\epsilon}}+|B u|_{2 ; p+1, T_{\epsilon}}+|h|_{1 ; 1, T_{\epsilon}}+|h|_{2 ; p+1, T_{\epsilon}}\right) .
\end{aligned}
$$

Consider $|h|_{1 ; 1, T_{\epsilon}}$. In place of (11.14) we have

$$
\left|\Phi \partial^{\langle j\rangle}\left(e^{-t} v_{0}\right) \partial^{\langle l\rangle} u \partial^{m} u \partial^{n} u\right|_{1 ; 0, T_{\epsilon}} .
$$

Instead of Lemma 10.2 just use Cauchy-Schwartz to obtain (11.21) $\leq$

$$
C\left|\partial^{m} u\right|_{2 ; 0, T_{\epsilon}}\left|\partial^{\langle j\rangle}\left(e^{-t} v_{0}\right) \partial^{\langle l\rangle} u \partial^{n} u\right|_{2 ; 0, T_{\epsilon}} \leq f\left(E_{p}\right) .
$$

$|B u|_{1 ; 1, T_{\epsilon}}$ is similar and (11.13) holds, so the proof of Theorem4.4 is finished.

$$
\text { 12. } L^{1}-L^{p} \text { estimates, } p \geq 2
$$

Henceforth we revert to the notation $\left(t, x_{1}, x^{\prime}\right)$ in place of $(t, x, y)$ and $\left(\tau, \xi_{1}, \xi^{\prime}\right)$ in place of $(\tau, \xi, \eta)$.

We next establish $L^{1}-L^{p}$ bounds for the conjugated doubled eigenvalue equation, $p \geq 2$. From here on, we assume the auxiliary structural hypothesis $(\mathrm{H} 6)$; that is, we assume that branch singularities of characteristic roots $\xi_{1}$ (considered as functions of $\left.\left(\tau, \xi^{\prime}\right)\right)$ are confined to a finite union of smooth surfaces $\tau=\tau_{j}\left(\xi^{\prime}\right)$ on which the singularity has constant order equal to $s_{j}$, the multiplicity of the corresponding root $\xi_{1}$. 
We work in polar coordinates $\left(\hat{\lambda}, \hat{\xi}^{\prime}, \rho\right)$ with $\hat{\gamma}:=\Re \hat{\lambda}, \hat{\tau}:=\Im \hat{\lambda}$. Departing somewhat from an earlier assumption, we now relax our standard requirement $\hat{\gamma} \geq 0$ to the more general

$$
\hat{\gamma} \geq-\theta \rho\left(|\hat{\tau}|^{2}+\left|\hat{\xi}^{\prime}\right|^{2}\right)
$$

for small frequencies and

$$
\hat{\gamma} \geq-\theta\left(|\hat{\tau}|+\rho\left|\hat{\xi}^{\prime}\right|^{2}\right)
$$

for mid- and high-frequencies, $\theta>0$ sufficiently small. Then the main result, to be established in the remainder of this section, is the following, where $L^{1}, L^{2}$ refer to $L^{1}\left(x_{1}\right), L^{2}\left(x_{1}\right)$ norms.

Proposition 12.1. Assume (H1), (H2), (H3), (H4), (H6), and (12.1). Then, for $F \in L^{1}$ and $\rho>0$ sufficiently small, the solution of the conjugated doubled boundary problem (6.34) satisfies

$$
|U|_{L^{2}}^{2} \leq \frac{C \beta^{2}|F|_{L^{1}}^{2}}{\rho^{2}}
$$

for some $C>0$ uniformly near the basepoint $X_{0}$, where

$$
\beta:=\max _{j \geq 0} \beta_{j}
$$

with $\beta_{0}:=1$ and

$$
\beta_{j}:=\left(\left|\hat{\tau}-\tau_{j}\left(\hat{\xi}^{\prime}\right)\right|+\rho+\hat{\gamma}\right)^{1 / s_{j}-1} .
$$

(Note that $\beta=1$ if the glancing set $\mathcal{G}$ is empty, in particular for $d=1$.)

From (12.3), we obtain readily the linear estimate (11.16) cited in the previous section.

Corollary 12.1. Assume (H1), (H2), (H3), (H4), and (H6). Then, for $d \geq 3$, the solution of the linear problem (11.2) (nonzero mass) satisfies

$$
\left|u, u_{y}, u_{t}\right|_{2 ; 0}+\left|u_{x}\right|_{2 ; 0} \leq C\left(|f|_{1 ; 0}+|f|_{2 ; 0}\right),
$$

while, for $d \geq 1$, the solution of (9.8) (zero mass) satisfies the same bound with $(F, G)$ in place of $f$.

Proof. Nonzero mass. We want to use the $L^{1} \rightarrow L^{2}$ bound (12.3) in exactly the same manner that the first inequality of (8.16) was used to establish the mixed norm estimate of Proposition 8.2. The key to doing so is the observation, which can be checked directly using the explicit form of $\beta_{j}$ given above, that for $\delta>0$

$$
\int_{\left|\tau, \xi^{\prime}\right|<\delta} \frac{\beta^{2}}{\rho^{2}} d \tau d \xi^{\prime}<\infty
$$

for dimension $d \geq 3$. (Some care is needed since $\beta$ is singular.)

Substituting (12.3) line by line for the first inequality of (8.16) in the proof of Proposition 8.2, we thus obtain (8.28) with pure $L^{1}$ norm $|f|_{L^{1}(x, t, y)}$ substituted for the mixed norm $|f|_{L^{2}\left(x, L^{1}(t, y)\right)}$ on the right-hand side. This concludes the treatment of the key small-frequency regime.

The treatment of the mid-frequency range goes exactly as before: since $\rho$ is bounded above and below, there is no difference between general forcing and $\rho$ forcing. The treatment of high frequencies, as noted in Section 8.1, is in fact 
somewhat simpler for general forcing. Combining these observations, we obtain the result.

Note that (12.7) fails for $d=1$ and 2. For this reason different arguments are needed to handle nonzero mass perturbations in these dimensions. The case $d=2$ is treated in Theorem 4.5. For $d=1$ see $[\mathrm{ZH}],[\mathrm{Z2}]$.

Zero mass. For " $\rho F$ "-forcing, we obtain in place of (12.3) the estimate

$$
|U|_{L^{2}}^{2} \leq C \beta^{2}|F|_{L^{1}}^{2}
$$

and $\beta^{2}$ is integrable near the origin for all $d \geq 1$. As in the proof of Theorem 4.1 one needs to consider an auxiliary problem to treat the $F_{x_{1}}$ part of the forcing. The small frequency estimate then follows almost exactly as for Theorem 4.1, with the $L^{1}\left(t, x_{1}, x^{\prime}\right)$ norm now playing the role of the mixed norm; see Lemma 12.6 of Section 12.6 below.

The treatment of mid- and high frequencies goes as before.

In what follows we will occasionally interpolate between $L^{2}$ and $L^{\infty}$ using the following elementary inequalities:

$$
|u|_{L^{p}} \leq|u|_{L^{\infty}}^{1-\frac{2}{p}}|u|_{L^{2}}^{\frac{2}{p}} \leq|u|_{L^{\infty}}+|u|_{L^{2}} .
$$

From (12.3) we obtain immediately the following $L^{1} \rightarrow L^{p}$ bounds, to be used in the next section.

Corollary 12.2. Assume (H1), (H2), (H3), (H4), (H6), and (12.1). Then, for $F \in L^{1}$ and $\rho>0$ sufficiently small, the solution of the conjugated doubled boundary problem (6.34) satisfies

$$
|u|_{L^{p}} \leq \frac{C \beta|F|_{L^{1}}}{\rho}
$$

for all $2 \leq p \leq \infty$, for some $C>0$ uniformly near the basepoint $X_{0}$, where $\beta$ is defined as in Proposition 12.1

Proof. Recall that $|U|$ bounds both $|u|$ and $\left|u_{x_{1}}\right|$. Thus, the result for $p=\infty$ follows from the standard one-dimensional Sobolev inequality

$$
|f|_{\infty} \leq|f|_{2}^{1 / 2}\left|f_{x_{1}}\right|_{2}^{1 / 2}
$$

and the general result $2 \leq p \leq \infty$ by interpolation between $L^{2}$ and $L^{\infty}$ norms.

Our basic strategy in proving Proposition 12.1 will be to establish an $L^{2} \rightarrow L^{\infty}$ bound for the adjoint problem and then to appeal to duality. In deriving adjoint $L^{2} \rightarrow L^{\infty}$ bounds, we use duality in a second way, to first conclude adjoint $L^{2} \rightarrow L^{2}$ bounds from the $L^{2} \rightarrow L^{2}$ bounds of the forward equation (slightly refined). From $L^{2}$ bounds on source and solution, $L^{\infty}$ bounds are then readily obtained by a standard energy estimate/integration by parts.

Remark 12.1. It is worth noting that we do not in this argument apply degenerate symmetrizers to the adjoint equation. Indeed, because of an asymmetry between forward vs. dual equations, our standard degenerate symmetrizer estimate would not recover the sharp bound available by duality. (Specifically, the degeneracy in the boundary condition for the dual equation occurs in hyperbolic modes, though we shall not show it here.) 
12.1. The dual problem. Consider a general boundary problem

$$
\begin{aligned}
& L U:=U_{x_{1}}-G\left(x_{1}, \lambda, \xi^{\prime}\right) U=F, \\
& \Gamma U=0 \text { on } x_{1}=0 .
\end{aligned}
$$

The dual problem is then defined via the $L^{2}$ inner product on $\mathbb{R}^{+}$as

$$
\begin{aligned}
& L^{*} V:=-V_{x_{1}}-G^{*}\left(x_{1}, \lambda, \xi^{\prime}\right) U=G, \\
& \Gamma^{*} V=0 \text { on } x_{1}=0,
\end{aligned}
$$

where the kernel of $\Gamma^{*}$ is the orthogonal complement of the kernel of $\Gamma$, i.e., by the property that

$$
\langle L U, V\rangle=\left\langle U, L^{*} V\right\rangle
$$

for $\Gamma U(0)=\Gamma^{*} V(0)=0$.

A formality is to first establish well-posedness of both problems.

Proposition 12.2. For $\rho>0$, both forward and dual problems have a unique $H^{1}$ solution for any data in $L^{2}$.

Proof. It is sufficient to prove uniqueness, which follows in both cases from the standard (nondegenerate) symmetrizer construction carried out for fixed $\rho \neq 0$. The interior estimates thereby obtained feature constants that may blow up arbitrarily fast in $\rho$ as $\rho \rightarrow 0$; however, this is of no consequence for the present purpose.

Corollary 12.3. The bound of Proposition 12.1 is equivalent to the dual bound

$$
|V|_{L^{\infty}}^{2} \leq \frac{C \beta^{2}}{\rho^{2}}|G|_{2}^{2}
$$

for solutions of the dual conjugated boundary problem, for $G \in L^{2}$.

Proof. We have

$$
|U|_{L^{2}}=\sup _{|G|_{L^{2}}=1}\langle U, G\rangle=\left\langle U, L^{*} V\right\rangle=\langle L U, V\rangle=\langle F, V\rangle \leq|F|_{L^{1}}|V|_{L^{\infty}},
$$

from which we obtain the forward direction

$$
|U|_{L^{2}} /|F|_{L^{1}} \leq|V|_{L^{\infty}} /|G|_{L^{2}}
$$

A reverse calculation yields the backward direction.

12.2. Decomposition of $U_{H \pm}$. To establish (12.15), we will need to sharpen the basic $L^{2} \rightarrow L^{2}$ estimate for the forward equation. To do this, we shall need to decompose the hyperbolic modes $U_{H}$ in decomposition (6.16) as the sum $U_{H}=$ $U_{H_{+}}+U_{H_{-}}$, where

$$
U_{H_{ \pm}}=U_{H_{h \pm}}+U_{H_{e \pm}}+U_{H_{g \pm}} .
$$

Each vector appearing in (12.18) has $4 n$ components, and the decomposition depends on $\left(\lambda, \xi^{\prime}, \rho\right)$. While $U_{H}$ here is the same as the vector $U_{H}$ appearing in (6.16), to avoid confusion it is important to note that the definitions of $U_{H_{ \pm}}$are different now as we explain below.

We shall write

$$
U_{H_{h}}=U_{H_{h+}}+U_{H_{h-}}
$$

and do similarly for $e$ and $g$. The hyperbolic mode $U_{H_{h \pm}}$ has nonvanishing components corresponding (only) to the blocks $Q_{k}$ in (6.10) which are $1 \times 1$ with real part 
vanishing at the basepoint, but with real part $>0($ resp. $<0)$ when $\rho>0$. The elliptic mode $U_{H_{e \pm}}$ has nonvanishing components corresponding to blocks with $\Re Q_{k}$ positive or negative definite at the basepoint. Finally, the glancing mode $U_{H_{g}}$ has nonvanishing components corresponding to blocks of size larger than $1 \times 1$ which are purely imaginary at the basepoint (glancing blocks).

Furthermore, we shall diagonalize the glancing blocks by a $4 n \times 4 n$ matrix $T_{H_{g}}\left(\lambda, \xi^{\prime}, \rho\right)$ :

$$
U_{H_{g}}^{\prime}:=T_{H_{g}}^{-1} U_{H_{g}},
$$

where $U_{H_{g}}:=U_{H_{g+}}+U_{H_{g-}}$. Here $U_{H_{g \pm}}$ are defined as the projections of $U_{H_{g}}$ onto the growing (resp. decaying) eigenspaces of $\hat{H}_{B}$ in (6.10) corresponding to glancing blocks. Call these subspaces $H_{g \pm}$. Clearly, $T_{H_{g}}$ also has a block structure and we may construct it so that in any given block corresponding to a glancing block $\mathcal{Q}_{j}$, the first columns are eigenvectors of $\mathcal{Q}_{j}$ associated (for $\rho>0$ ) to eigenvalues with $\Re \mu<0$. The remaining blocks of $T_{H_{q}}$ are identity matrices.

We denote by

$$
U^{\prime}:=T_{H_{g}}^{-1} U
$$

the full variable with $U_{H_{g}}$ diagonalized and all other components unchanged. By calculations similar to those in [Z1], we obtain the following estimates.

Lemma 12.1. The diagonalizing transformation $T_{H_{g}}$ may be chosen so that

$$
\begin{gathered}
\left|T_{H_{g}}\right| \leq C, \\
\left|T_{H_{g}}^{-1}\right| \leq C \beta,
\end{gathered}
$$

and

$$
\left|T_{H_{g \mid H_{g-}}}^{-1}\right| \leq C \alpha,
$$

where $\beta:=\max _{j} \beta_{j}, \alpha:=\max _{j} \alpha_{j}$, with

$$
\begin{gathered}
\beta_{j}:=\theta_{j}^{1-s_{j}}, \quad \alpha_{j}:=\theta_{j}^{1-\left[\left(s_{j}+1\right) / 2\right]}, \\
\theta_{j}:=\left(\left|\hat{\tau}-\tau_{j}\left(\hat{\xi}^{\prime}\right)\right|+\hat{\gamma}+\rho\right)^{1 / s_{j}},
\end{gathered}
$$

and $T_{H_{g \mid H_{g-}}^{-1}}^{-1}$ denotes the restriction of $T_{H_{g}}^{-1}$ to subspace $H_{g-}$. In particular,

$$
\beta \alpha^{-2} \geq 1 \text {. }
$$

Remark 12.2. The quantities $\beta$ and $\alpha$, and their sharp estimation, we regard as a key to the analysis of long-time stability in multidimensions.

Proof. Clearly, it is sufficient to establish for a single block $\mathcal{Q}_{j}$ of size $s_{j}$ that there exist diagonalizing matrices whose inverses are bounded by $\beta_{j}, \alpha_{j}$, respectively. Let $\underline{\mu}$ denote the multiple pure imaginary eigenvalue appearing in $\mathcal{Q}_{j}$ evaluated at the basepoint $\left(\underline{\hat{\tau}}, \underline{\hat{\xi}^{\prime}}\right)$. From here on, we drop the $j$ subscript.

Set $\sigma=\left(\left|\hat{\tau}-\tau\left(\hat{\xi}^{\prime}\right)\right|+\hat{\gamma}+\rho\right)$ so $\theta=(\sigma+\rho)^{1 / s}$. By a classic matrix perturbation argument (e.g., [Z1], Lemma 4.8) the eigenvalue $\underline{\mu}$ splits for $\sigma+\rho>0$ small into $s$ eigenvalues.

$$
\mu_{k}=\underline{\mu}+\pi_{k}+o\left(|\sigma, \rho|^{1 / s}\right), k=1, \ldots, s .
$$


Here

$$
\begin{aligned}
& \pi_{k}=\epsilon^{k} i(p \sigma-i q \rho)^{1 / s} \text { with } \\
& \epsilon=1^{1 / s}, \\
& p\left(\hat{\xi}^{\prime}\right) \text { and } q\left(\hat{\xi}^{\prime}\right) \text { are real and } \sim 1, \text { and } \operatorname{sgn} p=\operatorname{sgn} q .
\end{aligned}
$$

Moreover, corresponding eigenvectors are given in appropriate coordinates by

$$
\left(1, \pi_{k}, \pi_{k}^{2}, \ldots, \pi_{k}^{s-1}\right)+o\left(|\sigma, \rho|^{1 / s}\right) .
$$

Thus, there exists a matrix $T_{H_{q}}$ of eigenvectors of the $s \times s$ block $Q$ that is approximately given by a Vandermonde matrix with generators distance at least $\theta$ apart related by $s$ roots of unity. (Note: In [Z1], $\gamma$ was constrained as a function of $\tau, \rho$; however, an examination of the argument shows that the analysis remains valid in the more general case (12.1).)

By Kramer's rule, we may therefore estimate $\beta$ as the quotient of two Vandermonde determinants, the numerator of size $s-1$ and the denominator of size $s$, taken from the same set of equally spaced generators. The standard formula for Vandermonde determinants then gives

$$
\beta \sim \theta \theta^{\left(\begin{array}{c}
s-1 \\
2
\end{array}\right)-\left(\begin{array}{l}
s \\
2
\end{array}\right)}=\theta^{1-s}
$$

as claimed.

Denoting by

$$
\left(\begin{array}{l}
t_{1} \\
t_{2}
\end{array}\right)
$$

the matrix consisting of the $k \leq[(s+1) / 2]$ stable eigenvectors of $Q$, i.e., the first $k$ columns of $T_{H_{g}}$, and noting that $t_{1}$ as a Vandermonde matrix is invertible, we find that $H_{g-}$ consists of vectors of form

$$
\left(\begin{array}{c}
w \\
t_{2} t_{1}^{-1} w
\end{array}\right)=\left(\begin{array}{c}
t_{1} \\
t_{2}
\end{array}\right) t_{1}^{-1} w
$$

where $w \in \mathbb{C}^{k}$ is arbitrary.

From $\left|\left(w, t_{2} t_{1}^{-1} w\right)\right| \geq|w|$ and the computation

$$
\begin{aligned}
\left|T_{H_{g}}^{-1}\left(\begin{array}{c}
w \\
t_{2} t_{1}^{-1} w
\end{array}\right)\right| & =\left|\left(\begin{array}{cc}
t_{1} & * \\
t_{2} & *
\end{array}\right)^{-1}\left(\begin{array}{c}
t_{1} \\
t_{2}
\end{array}\right) t_{1}^{-1} w\right| \\
& =\left|\left(\begin{array}{c}
I_{k} \\
0
\end{array}\right) t_{1}^{-1} w\right| \\
& =\left|t_{1}^{-1} w\right|
\end{aligned}
$$

we thus obtain that $\left|T_{H_{g \mid H_{g-}}^{-1}}^{-1}\right| \leq\left|t_{1}^{-1}\right|$.

Observing that $t_{1}$ is a $k \times k$ Vandermonde matrix with generators taken from the same equally spaced set and applying Kramer's rule similarly as before, we obtain

$$
\left|t_{1}^{-1}\right| \leq C \theta^{1-[(s+1) / 2]}
$$

and thus $\alpha=\theta^{1-[(s+1) / 2]}$ as claimed.

We define similar decompositions on the dual variable $V$ and also the forcing terms $F$ and $G$. 
12.3. Interior estimates. We begin by carrying out a basic degenerate symmetrizer estimate for the diagonalized forward problem. Note that the treatment of glancing modes is considerably simpler in diagonalized coordinates and indeed has nothing to do with that of the original Kreiss construction.

Lemma 12.2. For the forward diagonalized problem, we have the interior bound

$$
\left|U^{\prime}\right|_{L^{2}}^{2} \leq C \frac{\left|F^{\prime}\right|_{L^{2}}^{2}}{\rho^{2}\left(\gamma+\rho^{2}\right)}
$$

Proof. In diagonalized coordinates, we must deal with a new degeneracy of order $\alpha^{-1}$ in the glancing modes of the diagonalized boundary condition $\Gamma^{\prime}:=\Gamma T_{H_{g}}$ for the forward problem, as may be seen by the calculation

$$
\left|\Gamma^{\prime} U_{H_{g-}}^{\prime}\right|=\left|\Gamma U_{H_{g-}}\right| \geq C^{-1}\left|U_{H_{g-}}\right| \geq \frac{C^{-1}\left|U_{H_{g-}}^{\prime}\right|}{\left|T_{H_{g \mid H_{g-}}}^{-1}\right|} .
$$

On the other hand, there are no coalescing modes, and so we may dispense with the usual Kreiss construction, treating glancing modes in the same way as hyperbolic and elliptic modes. Precisely, in all except glancing modes, we make the same choice of degenerate symmetrizer followed in previous sections, while in the glancing modes we choose

$$
S_{H_{g}}=\operatorname{diag}\left(S_{H_{g+}}, S_{H_{g-}}\right):=\operatorname{diag}\left(C, \alpha^{-2}\right) .
$$

Evidently, it holds that

$$
\left|\Gamma^{\prime} U_{-}^{\prime}\right| \geq C\left(\delta\left|U_{H_{h-}}^{\prime}\right|+\delta\left|U_{H_{e-}}^{\prime}\right|+\alpha^{-1}\left|U_{H_{g-}}^{\prime}\right|+\rho\left|U_{P-}^{\prime}\right|\right),
$$

analogous to Lemma [7.1, and therefore we again obtain good trace terms in the resulting symmetrizer estimate.

It remains to check that we retain good interior $\left(L^{2}\right)$ bounds. Let $\mu_{k \pm}$ denote the eigenvalue associated with the $k$ th mode of $U_{H_{g}}^{\prime}$. Taking the Taylor expansion of the expression (12.28) for $\pi_{k}$ about $\rho / \sigma=0$ yields

$$
\left|\Re \mu_{k \pm}\right| \geq C^{-1} \rho^{2} \beta
$$

whence we obtain from the fact that $\beta \alpha^{-2} \geq 1$ the lower bound

$$
\alpha^{-2}\left|\Re \mu_{k \pm}\right| \geq C^{-1} \rho^{2},
$$

and thereby the key interior estimate

$$
\left(\operatorname{Re} S G_{B}^{\prime}(\infty) U_{H_{g}}^{\prime}, U_{H_{g}}^{\prime}\right) \geq \alpha^{2} \rho^{2}\left|U_{H_{g+}}^{\prime}\right|_{2}^{2}+\rho^{2}\left|U_{H_{g-}}^{\prime}\right|_{2}^{2} .
$$

That is, we still find that $\Re S G_{B}^{\prime}(\infty) \geq \rho^{2}$ as before, and therefore the rest of the calculation of Section 8 goes through as before to give the claimed estimate.

Remark 12.3. Since $T_{H_{g}}$ diagonalizes the forward problem, $T_{H_{g}}^{-1 *}$ diagonalizes the dual problem.

By duality, this yields

Corollary 12.4. For the dual diagonalized problem, we have the interior bound

$$
\left|V^{\prime}\right|_{L^{2}}^{2} \leq \frac{C\left|G^{\prime}\right|_{L^{2}}^{2}}{\left(\gamma+\rho^{2}\right) \rho^{2}}
$$


In fact, the above estimates can be somewhat refined. Let $U_{H_{g \pm, j}}^{\prime}$ denotes the $j$ th growing/decaying glancing mode and $\mu_{j \pm}$ the associated growth/decay rate (eigenvalue of $G_{B}$ ).

Lemma 12.3. For the forward diagonalized problem, we have the refined interior bounds

$$
\left|U^{\prime}\right|_{2}^{2} \leq C \frac{\left|F_{P}\right|_{2}^{2}+\left(\gamma+\rho^{2}\right)^{-1}\left|F_{H_{h}}\right|_{2}^{2}+\rho^{-1}\left|F_{H_{e}}\right|_{2}^{2}+\sum_{j, \pm}\left|\Re \mu_{j \pm}\right|^{-1}\left|F_{H_{g \pm, j}}\right|_{2}^{2}}{\rho^{2}} .
$$

Proof. Parabolic modes have growth/decay rates with real part bounded in absolute value above and below by order one; elliptic modes have growth/decay rates bounded above and below by order $\rho$; hyperbolic modes have growth/decay rates bounded above and below by order $\left(\gamma+\rho^{2}\right)$. Glancing modes are treated individually in the diagonalized coordinates and have growth/decay rates with absolute value of real part $\left|\Re \mu_{j \pm}\right|$. Using this sharp information in the degenerate symmetrizer estimate described just above, specifically in the application of Young's inequality in step (8.10) of Section 8 we obtain the claimed bound. Note that the worst-case version of (12.43) is (12.2), corresponding to component $F_{H_{h}}$.

Corollary 12.5. For the dual diagonalized problem, we have the interior bounds

$$
\left|V_{P}^{\prime}\right|_{L^{2}}^{2}+\left(\gamma+\rho^{2}\right)\left|V_{H_{h}}^{\prime}\right|_{L^{2}}^{2}+\rho\left|V_{H_{e}}^{\prime}\right|_{L^{2}}^{2}+\sum_{j \pm}\left|\Re \nu_{j \pm}\right|\left|V_{H_{g \pm, j}}^{\prime}\right|_{L^{2}}^{2} \leq \frac{C\left|G^{\prime}\right|_{L^{2}}^{2}}{\rho^{2}}
$$

where $\nu_{j \pm}=-\mu_{j \mp}^{*}$ denote growth/decay rates for the dual problem (eigenvalues of $\left.-G_{B}^{*}\right)$.

Proof. Perform integration by parts, exactly as in the proof of Corollary 12.3, but mode by mode. For example, to obtain the bound

$$
\rho\left|V_{H_{e}}^{\prime}\right|_{L^{2}}^{2} \leq \frac{C\left|G^{\prime}\right|_{L^{2}}^{2}}{\rho^{2}}
$$

we begin with bound

$$
\rho\left|U^{\prime}\right|_{L^{2}}^{2} \leq C \rho^{-2}\left|F_{H_{e}}^{\prime}\right|_{L^{2}}^{2}
$$

for the forward problem $L^{\prime} U^{\prime}=F_{H_{e}}^{\prime}$, and we calculate

$$
\begin{aligned}
\left|V_{H_{e}}^{\prime}\right|_{L^{2}} & =\sup _{\left|F_{H_{e}}^{\prime}\right|=1}\left\langle V_{H_{e}}^{\prime}, F_{H_{e}}^{\prime}\right\rangle=\sup \left\langle V^{\prime}, L^{\prime} U^{\prime}\right\rangle=\sup \left\langle L^{\prime}{ }^{\prime} V^{\prime}, U^{\prime}\right\rangle \\
& =\sup \left|G^{\prime}\right|_{L^{2}}\left|U^{\prime}\right|_{L^{2}} \leq\left|G^{\prime}\right|_{L^{2}} C \rho^{-3 / 2}\left|F_{H_{e}}^{\prime}\right|_{L^{2}} \\
& =C \rho^{-3 / 2}\left|G^{\prime}\right|_{L^{2}} .
\end{aligned}
$$

12.4. $L^{\infty}$ estimates. With these preparations, $L^{\infty}$ estimates are now easily obtained.

Lemma 12.4. For the dual problem, we have the bounds

$$
\left|V^{\prime}\right|_{\infty}^{2} \leq \frac{C\left|G^{\prime}\right|_{L^{2}}^{2}}{\rho^{2}}, \quad|V|_{\infty}^{2} \leq \frac{C \beta^{2}|G|_{L^{2}}^{2}}{\rho^{2}}
$$


Proof. Working in diagonalized coordinates, we may take the real part of the $L^{2}$ inner product of $V^{\prime}$ with equation $\left(L^{\prime}\right)^{*} V^{\prime}=G^{\prime}$ from $x_{0} \geq 0$ to plus infinity to obtain after integration by parts the estimate

$$
\begin{aligned}
& \left|V^{\prime}\left(x_{0}\right)\right|^{2} \leq C\left(\left|V_{P}^{\prime}\right|_{2}^{2}+\left(\gamma+\rho^{2}\right)\left|V_{H_{h}}^{\prime}\right|_{2}^{2}+\rho\left|V_{H_{e}}^{\prime}\right|_{2}^{2}+\sum_{j \pm}\left|\Re \nu_{j}\right|\left|V_{H_{g \pm, j}}^{\prime}\right|_{2}^{2}\right) \\
& +C\left|V^{\prime}\right|_{2}\left|G^{\prime}\right|_{2} .
\end{aligned}
$$

Bounding the first term on the right-hand side using Corollary 12.5] and the second term using Corollary 12.4, we obtain the first asserted bound. The second asserted bound then follows by change of coordinates and the Jacobian bounds of Lemma 12.1 .

This completes the proof of Proposition 12.1.

Remark 12.4. (1) Note that no symmetrizer construction was carried out for the dual problem, to obtain neither interior nor trace estimates; indeed, our degenerate symmetrizer construction applied to the dual problem does not seem to yield the sharp $L^{2}$ bounds we obtain by reference to the forward problem. A review of the argument structure shows that the approach is completely general, in the sense that it will always yield some $L^{2}$ estimate for the dual diagonalized equations and an $L^{\infty}$ bound improving on that bound by a factor equal to the minimum growth rate among all modes.

(2) The resolvent bound derived here agrees with that obtained by integration of the pointwise bounds stated in Proposition 4.5 of [Z1]; however, as noted in [Z1], slightly better bounds were in fact established, and these yield (on integration) the improved bound $|U|_{2}^{2} \leq \frac{C \beta|F|_{1}^{2}}{\rho}$; see Remark 4.35 of [Z1]. As pointed out in the same remark, this improved bound is sharp for square root singularities, $s=2$, but likely not for higher order branch singularities.

(3) It would be very interesting to determine analogous bounds in the situation that (H6) does not hold. Let us denote the resulting factor of singularity as $\tilde{\beta}(d, s)$, depending on dimension $d$ and maximum order of singularity $s$. Simple examples show that $\tilde{\beta}(d, s)>>\beta(s)$; however, we conjecture that $\tilde{\beta}(d, s)^{2}$ nonetheless remains integrable in $R^{d}$, for all fixed $d$ and $s$, as needed for our arguments.

12.5. Mid- and high-frequency estimates. In the next section, we shall need also the following straightforward bounds.

Lemma 12.5. Assume (H1), (H2), (H3), and (H4). Then, for $F \in L^{2}$ and $\rho$ bounded uniformly above and below, and under assumption (12.2), the solution of the conjugated doubled boundary problem (6.34) satisfies

$$
|u|_{2}^{2}+\left|u_{x_{1}}\right|_{2}^{2}+\left|u_{x_{1} x_{1}}\right|_{2}^{2} \leq C|F|_{2}^{2}
$$

for some $C>0$. For $\rho$ sufficiently large, and under assumption (12.2), the solution satisfies (in polar coordinates, suppressing hats)

$$
\left(\rho|\tau|+\rho \gamma+\rho^{2}\left|\xi^{\prime}\right|^{2}\right)^{2}|u|_{2}^{2}+\left(\rho|\tau|+\rho \gamma+\rho^{2}\left|\xi^{\prime}\right|^{2}\right)\left|u_{x_{1}}\right|_{2}^{2}+\left|u_{x_{1} x_{1}}\right|_{2}^{2} \leq C|F|_{2}^{2}
$$

Proof. The $|u|_{2}$ and $\left|u_{x_{1}}\right|_{2}$ bounds follow by essentially the same calculation as in the proof of Proposition 9.1, but substituting general forcing $F$ in place of divergence forcing $F_{x_{1}}+i \eta G$. The $\left|u_{x_{1} x_{1}}\right|_{2}$ bounds can then be obtained directly from the equation (9.1). 
Corollary 12.6. Assume (H1), (H2), (H3), and (H4). Then, for $F \in L^{1}, \rho$ bounded uniformly above and below and under assumption (12.2), the solution of the conjugated doubled boundary problem (6.34) satisfies

$$
|u|_{2}+\left|u_{x_{1}}\right|_{2} \leq C|F|_{1}
$$

for some $C>0$. For $\rho$ sufficiently large, and under assumption (12.2), the solution satisfies (in polar coordinates, suppressing hats)

$$
\left(\rho|\tau|+\rho \gamma+\rho^{2}\left|\xi^{\prime}\right|^{2}\right)^{3 / 4}|u|_{2}+\left(\rho|\tau|+\rho \gamma+\rho^{2}\left|\xi^{\prime}\right|^{2}\right)^{1 / 4}\left|u_{x_{1}}\right|_{2} \leq C|F|_{1} .
$$

Proof. By duality, the bounds (12.52) and (12.53) hold also for the adjoint equation. Applying the one-dimensional Sobolev inequality $|f|_{\infty} \leq|f|_{2}^{1 / 2}\left|f_{x_{1}}\right|_{2}^{1 / 2}$, we thus obtain the adjoint $L^{2} \rightarrow L^{\infty}$ bounds

$$
|v|_{\infty}+\left|v_{x_{1}}\right|_{\infty}+\left|v_{x_{1} x_{1}}\right|_{\infty} \leq C|G|_{2}
$$

for $\rho$ bounded above and below, and

$$
\left(\rho|\tau|+\rho \gamma+\rho^{2}\left|\xi^{\prime}\right|^{2}\right)^{3 / 4}|v|_{\infty}+\left(\rho|\tau|+\rho \gamma+\rho^{2}\left|\xi^{\prime}\right|^{2}\right)^{1 / 4}\left|v_{x_{1}}\right|_{\infty} \leq C|G|_{2}
$$

for $\rho$ sufficiently large, from which the claimed bounds follow by duality.

Corollary 12.7. Assume (H1), (H2), (H3), and (H4). Then, for $F \in L^{1}, \rho$ bounded uniformly above and below, and under assumption (12.2), the solution of the conjugated doubled boundary problem (6.34) satisfies

$$
|u|_{p} \leq C|F|_{1}
$$

for all $2 \leq p \leq \infty$, for some $C>0$. For $\rho$ sufficiently large, and under assumption (12.2), the solution satisfies (in polar coordinates, suppressing hats)

$$
\left(\rho|\tau|+\rho \gamma+\rho^{2}\left|\xi^{\prime}\right|^{2}\right)^{1 / 2+1 / 2 p}|u|_{p} \leq C|F|_{1} .
$$

Proof. As in the proof of Corollary 12.2, this follows immediately by one-dimensional Sobolev inequality and interpolation.

Remark 12.5. Comparison with the explicit resolvent bounds of [Z1] shows that the above estimates are sharp.

12.6. The auxiliary problem. Finally, we point out the following straightforward estimates for auxiliary problem (10.3).

Lemma 12.6. Assuming (H2)-(H3), there exists a solution $\hat{w}$ of auxiliary problem (10.3) satisfying

$$
|\hat{w}|_{L^{p}\left(x_{1}\right)} \leq C|\hat{F}|_{L^{1}\left(x_{1}\right)}
$$

for all $1 \leq p \leq \infty$, with $C>0$ independent of $p$.

Proof. As before, we begin by conjugating to a constant coefficient doubled boundary value problem and imposing the augmented boundary condition $\tilde{B}$. Since the eigenvalues of $A$ are nonzero, real, and distinct, we can further conjugate by a constant matrix to the case that $\mathcal{A}(\infty)$ is diagonal, and $w$ may be decomposed entirely into scalar components $w_{j}$. Integrating the vector $\left(\operatorname{sgn} w_{j}\right)$ against $S$ times the diagonalized equation, where $S$ is the usual symmetrizer, we thus obtain the estimate

$$
C^{-1}\left|\hat{w}_{+}(0)\right|-\left|\tilde{B} \hat{w}_{-}(0)\right|+|\hat{w}|_{L^{1}\left(x_{1}\right)} \leq|\hat{F}|_{L^{1}\left(x_{1}\right)},
$$


yielding the desired estimate for $p=1$. Next, taking the inner product of $\hat{w}$ against the original equation (10.3) and integrating from $x_{0}$ to infinity, we obtain

$$
\left|\hat{w}\left(x_{0}\right)\right|^{2} \leq C\left(|\hat{w}|_{L^{2}\left(x_{1}\right)}^{2}+\langle\hat{w}, \hat{F}\rangle\right),
$$

and therefore, by Hölder's inequality,

$$
|\hat{w}|_{L^{\infty}\left(x_{1}\right)}^{2} \leq C\left(|\hat{w}|_{L^{1}\left(x_{1}\right)}|\hat{w}|_{L^{\infty}\left(x_{1}\right)}+|\hat{w}|_{L^{\infty}\left(x_{1}\right)}|\hat{F}|_{L^{1}\left(x_{1}\right)}\right) .
$$

Dividing both sides by $|\hat{w}|_{L^{\infty}\left(x_{1}\right)}$ and applying bound (12.61), we obtain the result for $p=\infty$. The remaining bounds then follow by interpolation between $p=1$ and $p=\infty$.

\section{NONLINEAR Stability FOR $d=2$}

The nonlinear iteration scheme of Section 11 fails for nonzero mass perturbations in dimension $d=2$. On the one hand the proof fails, since $\beta^{2} / \rho^{2}$ is not integrable then. But this reflects the underlying fact that the linearized response to nonzero mass $L^{1}$ initial data in general decays in $L^{p}, p \geq 2$, no faster than a $d$-dimensional heat kernel. Though not explicitly stated in [Z1], this is a consequence of the bounds therein, which show that far field behavior is dominated by the outgoing portion of a "multidimensional diffusion wave," in the sense of [HoZ1]; examination of the (upper and lower) bounds of HoZ1 in the specific case of compressible NavierStokes equations then yields the result. Likewise, review of the nonlinear iteration scheme of [Z1] shows that this linear response is the dominant part of the solution, and therefore similar bounds hold for the full, nonlinear solution $U$. In particular, $|U|_{2}(t) \sim(1+t)^{-d / 4}$, and thus

$$
|U|_{L^{2}(x, t)}^{2}=\int_{0}^{\infty}|U|_{2}^{2}(t) d t \sim \int_{0}^{\infty}(1+t)^{-d / 2} d t
$$

converges if and only if $d \geq 3$. Since convergence of the iteration scheme implies $|U|_{L^{2}(x, t)}<+\infty$, we find that the scheme cannot work for $d \leq 2$, except for zero mass initial data, where it works for $L^{1} \cap H^{s}$ initial data for all $d \geq 1$, Theorem 4.2 .

In this section, we show that the resolvent bounds we have derived are nonetheless sufficient to yield a nonzero mass stability result for $d \geq 2$, by following a different approach introduced in [Z1]. This argument has the advantage of yielding at the same time rates of decay, thus improving the previous results also for $d \geq 3$; recall, the $[\mathrm{KK}]$-type scheme yields decay with no rate. These rates, however, are not expected to be sharp in the uniformly stable case considered here; see discussion below Proposition 8.1 in [ZS] or in Section 3.3. of [Z1].

13.1. Linear estimates. Define by $\Phi(t)$ the solution operator for the linearized Cauchy problem

$$
\begin{aligned}
& \text { (a) } u_{t}+\left(A\left(x_{1}\right) u\right)_{x_{1}}+\sum_{j=2}^{d} A_{j}\left(x_{1}\right) \partial_{j} u-\triangle u=0, \\
& \text { (b) }\left.u\right|_{t=0}=f .
\end{aligned}
$$

The main step is then to establish the following bounds (Proposition 4.45 of [Z1]). 
Proposition 13.1. Assume (H1), (H2), (H3), (H4), and (H6). Then the bounds

$$
\begin{aligned}
|\Phi(t) f|_{p} & \leq C(1+t)^{-\frac{d-1}{2}\left(1-\frac{1}{p}\right)}\left(\|f\|_{1}+\|f\|_{p}\right), \\
\left|\Phi(t) D_{x_{j}} f\right|_{p} & \leq C t^{-\frac{1}{2}}(1+t)^{-\frac{d-1}{2}\left(1-\frac{1}{p}\right)}\left(\|f\|_{1}+\|f\|_{p}\right)
\end{aligned}
$$

hold for all $2 \leq p \leq \infty$. (Note: Here, all norms are with respect to spatial variables only.)

Proof. Standard short-time theory yields, for $t \leq 1$, the bounds

$$
\begin{gathered}
|\Phi(t) f|_{2} \leq C\|f\|_{2}, \\
\left|\Phi(t) D_{x_{j}} f\right|_{2} \leq C t^{-\frac{1}{2}}\|f\|_{2} .
\end{gathered}
$$

Thus, it is sufficient to establish, for $t \geq 1$, the bounds

$$
\begin{gathered}
|\Phi(t) f|_{p} \leq C t^{-\frac{d-1}{2}\left(1-\frac{1}{p}\right)}\|f\|_{1}, \\
\left|\Phi(t) D_{x_{j}} f\right|_{2} \leq t^{-\frac{1}{2}} t^{-\frac{d-1}{2}\left(1-\frac{1}{p}\right)}\|f\|_{1} .
\end{gathered}
$$

To this end, define contours $\Gamma\left(\xi^{\prime}\right)$ by

$$
\Re \lambda=-\theta_{1}|\Im \lambda|^{2}-\theta_{1}\left|\xi^{\prime}\right|^{2}
$$

for $|\Im \lambda| \leq R$ and

$$
\Re \lambda=-\theta_{1} R|\Im \lambda|-\theta_{1}\left|\xi^{\prime}\right|^{2}
$$

for $|\Im \lambda| \geq R$, with $\theta_{1}$ sufficiently small. Then, standard semigroup theory together with the resolvent bounds previously obtained gives the representation

$$
u(x, t ; y)=\frac{1}{(2 \pi i)^{d}} \int_{\xi^{\prime} \in \mathbb{R}^{d-1}} \oint_{\lambda \in \tilde{\Gamma}\left(\xi^{\prime}\right)} e^{i \xi^{\prime} \cdot x^{\prime}} e^{\lambda t} \hat{u}\left(x_{1}, \xi^{\prime}, \lambda\right) d \lambda d \xi^{\prime}
$$

for the solution $u$ of the linearized Cauchy problem, where $\hat{u}$ denotes the solution of the generalized resolvent equation obtained formally by the Laplace-Fourier transform; see the related Lemma 4.39 of [Z1].

This formal equation is just (using Duhamel's principle to replace Cauchy initial data by homogeneous initial data with forcing $f(x) \delta(t), \delta(\cdot)$ denoting the Dirac delta-function)

$$
\hat{u}_{x_{1} x_{1}}-\left(A_{1}\left(x_{1}\right) \hat{u}\right)_{x_{1}}-s\left(x_{1}, \lambda, \xi^{\prime}\right) \hat{u}=\tilde{f}\left(x_{1}, \xi^{\prime}\right),
$$

where $\tilde{f}$ denotes the Fourier transform of $f,\left(\tau, \xi^{\prime}\right)$ is dual to $\left(t, x^{\prime}\right), \lambda=i \tau+\gamma$ with (12.1), (12.2) and as usual

$$
s\left(x_{1}, \lambda, \xi^{\prime}\right)=\sum_{j=2}^{d} A_{j}\left(x_{1}\right) i \xi_{j}+\lambda I+\left|\xi^{\prime}\right|^{2} I .
$$

Bounding

$$
|\tilde{f}|_{L^{\infty}\left(\xi^{\prime}, L^{1}\left(x_{1}\right)\right)} \leq|f|_{L^{1}\left(x_{1}, x^{\prime}\right)}=|f|_{1}
$$

using Hausdorff-Young's inequality and appealing to the $L^{1} \rightarrow L^{p}$ resolvent estimates of the previous section, we may thus bound

$$
\left|\hat{u}\left(x_{1}, \xi^{\prime}, \lambda\right)\right|_{L^{p}\left(x_{1}\right)} \leq|f|_{1} b\left(\xi^{\prime}, \lambda\right),
$$

where, for $\rho:=\left|\xi^{\prime}\right|+|\lambda|$, and $R>0$ sufficiently large, $b:=C \beta \rho^{-1}$ for $\rho \leq 1 / R$, $b:=C$ for $1 / R \leq \rho \leq R$, and $b:=C\left(|\lambda|+\left|\xi^{\prime}\right|^{2}\right)^{-1 / 2-1 / 2 p}$ for $\rho>R$. 
$L^{2}$ bounds. Using in turn Parseval's identity, Fubini's Theorem, the triangle inequality, and our $L^{1} \rightarrow L^{2}$ resolvent bounds, we may estimate

$$
\begin{aligned}
|u|_{L^{2}\left(x_{1}, x^{\prime}\right)}(t) & =\left(\int_{x_{1}} \int_{\xi^{\prime} \in \mathbb{R}^{d-1}}\left|\oint_{\lambda \in \tilde{\Gamma}\left(\xi^{\prime}\right)} e^{\lambda t} \hat{u}\left(x_{1}, \xi^{\prime}, \lambda\right) d \lambda\right|^{2} d \xi^{\prime} d x_{1}\right)^{1 / 2} \\
& =\left(\int_{\xi^{\prime} \in \mathbb{R}^{d-1}}\left|\oint_{\lambda \in \tilde{\Gamma}\left(\xi^{\prime}\right)} e^{\lambda t} \hat{u}\left(x_{1}, \xi^{\prime}, \lambda\right) d \lambda\right|_{L^{2}\left(x_{1}\right)}^{2} d \xi^{\prime}\right)^{1 / 2} \\
& \leq\left(\left.\left.\int_{\xi^{\prime} \in \mathbb{R}^{d-1}}\left|\oint_{\lambda \in \tilde{\Gamma}\left(\xi^{\prime}\right)}\right| e^{\lambda t}|| \hat{u}\left(x_{1}, \xi^{\prime}, \lambda\right)\right|_{L^{2}\left(x_{1}\right)} d \lambda\right|^{2} d \xi^{\prime}\right)^{1 / 2} \\
\leq & |f|_{1}\left(\int_{\xi^{\prime} \in \mathbb{R}^{d-1}}\left|\oint_{\lambda \in \tilde{\Gamma}\left(\xi^{\prime}\right)} e^{\Re \lambda t} b\left(\xi^{\prime}, \lambda\right) d \lambda\right|^{2} d \xi^{\prime}\right)^{1 / 2},
\end{aligned}
$$

from which we readily obtain the claimed bound on $|\Phi(t) f|_{2}$ using the bounds on $b$ on each of the small-, mid-, and high-frequency regions. For example, on the critical small-frequency region, parametrizing $\Gamma\left(\xi^{\prime}\right)$ by

$$
\lambda\left(\xi^{\prime}, k\right)=i k-\theta_{1}\left(k^{2}+\left|\xi^{\prime}\right|^{2}\right), \quad k \in \mathbb{R},
$$

and observing that in nonpolar coordinates

$$
\begin{aligned}
\rho^{-1} \beta & \leq\left[\left(|k|+\left|\xi^{\prime}\right|\right)^{-1}\left(1+\sum_{j \geq 1}\left(\frac{\left|k-\tau_{j}\left(\xi^{\prime}\right)\right|}{\rho}\right)^{\frac{1}{s_{j}}-1}\right)\right] \\
& \leq\left[\left(|k|+\left|\xi^{\prime}\right|\right)^{-1}\left(1+\sum_{j \geq 1}\left(\frac{\left|k-\tau_{j}\left(\xi^{\prime}\right)\right|}{\rho}\right)^{\varepsilon-1}\right)\right],
\end{aligned}
$$

where $\varepsilon:=\frac{1}{\max _{j} s_{j}}(0<\varepsilon<1$ chosen arbitrarily if there are no singularities), we obtain a contribution bounded by

$$
\begin{gathered}
C|f|_{1}\left(\int_{\xi^{\prime} \in \mathbb{R}^{d-1}}\left|\int_{-\infty}^{+\infty} e^{-\theta\left(k^{2}+\left|\xi^{\prime}\right|^{2}\right) t}(\rho)^{-1} \beta d k\right|^{2} d \xi^{\prime}\right)^{1 / 2} \\
\leq C|f|_{1} \int_{\xi^{\prime} \in \mathbb{R}^{d-1}}\left(\left.\left.e^{-2 \theta\left|\xi^{\prime}\right|^{2} t}\left|\xi^{\prime}\right|^{-2 \varepsilon}\left|\int_{-\infty}^{+\infty} e^{-\theta|k|^{2} t}\right| k\right|^{\varepsilon-1} d k\right|^{2} d \xi^{\prime}\right)^{1 / 2} \\
+C \sum_{j \geq 1}|f|_{1} \int_{\xi^{\prime} \in \mathbb{R}^{d-1}}\left(e^{-2 \theta\left|\xi^{\prime}\right|^{2} t}\left|\xi^{\prime}\right|^{-2 \varepsilon}\left|\int_{-\infty}^{+\infty} e^{-\theta|k|^{2} t}\right| k-\left.\left.\tau_{j}\left(\xi^{\prime}\right)\right|^{\varepsilon-1} d k\right|^{2} d \xi^{\prime}\right)^{1 / 2} \\
\leq C|f|_{1} \int_{\xi^{\prime} \in \mathbb{R}^{d-1}}\left(\left.\left.e^{-2 \theta\left|\xi^{\prime}\right|^{2} t}\left|\xi^{\prime}\right|^{-2 \varepsilon}\left|\int_{-\infty}^{+\infty} e^{-\theta|k|^{2} t}\right| k\right|^{\varepsilon-1} d k\right|^{2} d \xi^{\prime}\right)^{1 / 2} \\
\leq C|f|_{1} t^{-(d-1) / 4}
\end{gathered}
$$

as claimed.

To obtain the claimed bounds on $\left|\Phi(t) D_{x} f\right|_{2}$, we may use again the auxiliary problem (10.3) and the bounds of Lemma 12.6 to obtain for bounded frequencies the improved $L^{1} \rightarrow L^{2}$ bounds available for $\rho$-forcing, and thereby an additional factor of $\rho$ on the critical small-frequency region, which yields an additional factor of $t^{-1 / 2}$ in the estimate just above. On high-frequency regions, the estimate degrades by an algebraic factor in $\xi^{\prime}, \lambda$, but this is harmless for $t \geq 1$. 
$L^{\infty}$ bounds. Similarly, using Hausdorff-Young's inequality, we may estimate

$$
\begin{aligned}
|u|_{L^{\infty}\left(x_{1}, x^{\prime}\right)}(t) & \leq \sup _{x_{1}} \int_{\xi^{\prime} \in \mathbb{R}^{d-1}}\left|\oint_{\lambda \in \tilde{\Gamma}\left(\xi^{\prime}\right)} e^{\lambda t} \hat{u}\left(x_{1}, \xi^{\prime}, \lambda\right) d \lambda\right| d \xi^{\prime} \\
& \leq \int_{\xi^{\prime} \in \mathbb{R}^{d-1}} \oint_{\lambda \in \tilde{\Gamma}\left(\xi^{\prime}\right)}\left|e^{\lambda t}\right|\left|\hat{u}\left(x_{1} \xi^{\prime}, \lambda\right)\right|_{L^{\infty}\left(x_{1}\right)} d \lambda d \xi^{\prime} \\
& \leq|f|_{1} \int_{\xi^{\prime} \in \mathbb{R}^{d-1}} \oint_{\lambda \in \tilde{\Gamma}\left(\xi^{\prime}\right)} e^{\Re \lambda t} b\left(\xi^{\prime}, \lambda\right) d \lambda d \xi^{\prime}
\end{aligned}
$$

to obtain the claimed bound on $|\Phi(t) f|_{\infty}$. For example, on the critical smallfrequency region, parametrizing $\Gamma\left(\xi^{\prime}\right)$ again by

$$
\lambda\left(\xi^{\prime}, k\right)=i k-\theta_{1}\left(k^{2}+\left|\xi^{\prime}\right|^{2}\right), \quad k \in \mathbb{R},
$$

we obtain a contribution bounded by

$$
\begin{aligned}
& C|f|_{1} \int_{\xi^{\prime} \in \mathbb{R}^{d-1}} \int_{-\infty}^{+\infty} e^{-\theta\left(k^{2}+\left|\xi^{\prime}\right|^{2}\right) t} \rho^{-1} \beta d k d \xi^{\prime} \\
& \leq C|f|_{1} \int_{\xi^{\prime} \in \mathbb{R}^{d-1}} e^{-\theta\left|\xi^{\prime}\right|^{2} t}\left|\xi^{\prime}\right|^{-\varepsilon} \int_{-\infty}^{+\infty} e^{-\theta|k|^{2} t}|k|^{\varepsilon-1} d k d \xi^{\prime} \\
& \leq C|f|_{1} t^{-(d-1) / 2}
\end{aligned}
$$

as claimed. The improved bound on $\left|\Phi(t) D_{x} f\right|_{\infty}$ follows as before upon substitution of the improved $L^{1} \rightarrow L^{\infty}$ bounds available for $\rho$-forcing, exactly as in the case $p=2$.

General $2 \leq p \leq \infty$. Finally, the general case follows by interpolation between $L^{2}$ and $L^{\infty}$ norms.

13.2. Nonlinear stability. Nonlinear stability now follows by the argument of Proposition 4.46 of [Z1], for completeness reproduced here.

Proof of Theorem 4.5. Defining

$$
v:=u-\psi,
$$

and taking the Taylor expansion as usual, we obtain the nonlinear perturbation equation

$$
v_{t}-L v=\sum_{j} Q^{j}(v, v)_{x_{j}}
$$

where

$$
Q^{j}(v, v)=O\left(|v|^{2}\right)
$$

so long as $|v|$ remains bounded by some fixed constant. Applying Duhamel's principle and integrating by parts, we can thus express (supressing $x$-dependence)

$$
v(t)=\Phi(t) v(0)+\int_{0}^{t} \Phi(t-s) D_{x_{j}} Q^{j}(s) d s .
$$

Define now

$$
\zeta(t):=\sup _{0 \leq s \leq t, 2 \leq p \leq \infty}\|v(\cdot, s)\|_{L^{p}}(1+s)^{\frac{d-1}{4}} .
$$


We shall establish

Claim.

$$
\zeta(t) \leq C_{2}\left(\zeta_{0}+\zeta(t)^{2}\right)
$$

From this result, it follows by continuous induction that $\zeta(t) \leq 2 C_{2} \zeta_{0}$ for $t \geq 0$, provided $\zeta_{0}<1 / 4 C_{2}$. But definition (13.22) then yields

$$
\|v(\cdot, t)\|_{L^{2}} \leq 2 C_{2} \zeta(1+t)^{-\left(\frac{d-1}{4}\right)}
$$

as claimed. Thus, it remains, first, to establish the claim above, and, second, to extend to $2<p \leq \infty$.

Proof of Claim. Recalling (13.20) and (13.22), we can bound

$$
\begin{aligned}
\left|Q^{j}(v, v)(\cdot, t)\right|_{L^{p}} & \leq|v|_{L^{\infty}}|v|_{L^{p}} \\
& \leq C \zeta(t)^{2}(1+t)^{-\frac{d-1}{2}}
\end{aligned}
$$

for $p \geq 2$ and

$$
\begin{aligned}
\left|Q^{j}(v, v)(\cdot, t)\right|_{L^{1}} & \leq|v|_{L^{2}}^{2} \\
& \leq C \zeta(t)^{2}(1+t)^{-\frac{d-1}{2}},
\end{aligned}
$$

so that

$$
\left|Q^{j}(v, v)(\cdot, t)\right|_{L^{p}}+\left|Q^{j}(v, v)(\cdot, t)\right|_{L^{1}} \leq C \zeta(t)^{2}(1+t)^{-\frac{d-1}{2}} .
$$

The requisite $L^{\infty}$ bounds hold for a short time provided they hold initially, by local existence/regularity theory, and at later times provided that the $L^{\infty}$ bounds of the iteration scheme remain valid. Thus, we can establish the global validity of bounds (13.25) at the same time that we establish the global bound (13.24) on our iteration scheme, using the standard strategy of continuation.

Substituting into (13.21) and using bounds (13.3) and (13.4), we obtain

$$
\begin{aligned}
|v(t)|_{L^{p}} & \leq C \zeta_{0} t^{-\frac{d-1}{2}\left(1-\frac{1}{p}\right)} \\
& +C \zeta(t)^{2} \int_{0}^{t}(1+t-s)^{-\frac{d-1}{2}\left(1-\frac{1}{p}\right)}(t-s)^{-\frac{1}{2}}(1+s)^{-\frac{d-1}{2}} d s \\
& \leq C(p)\left(\zeta_{0}+\zeta(t)^{2}\right)(1+t)^{-\frac{d-1}{2}\left(1-\frac{1}{p}\right)},
\end{aligned}
$$

for all $2 \leq p \leq \infty$, where $C(p)$ is strictly monotone increasing in $p$, with $C(\infty)$ bounded for $d \geq 3$ and $C(\infty)=+\infty$ for $d=2$. (This final inequality follows by a direct calculation; see, e.g., Appendix A5 in [Z1.)

Of course, the integral in the second to last line is monotone decreasing in $p$, and so we may always substitute the less precise bound

$$
|v(t)|_{L^{p}} \leq C(2)\left(\zeta_{0}+\zeta(t)^{2}\right)(1+t)^{-\frac{d-1}{4}} .
$$

Thus, $\zeta(t) \leq C\left(\zeta_{0}+\zeta(t)^{2}\right)$, establishing the claim and the result for $p=2$. Once $\zeta$ is bounded, (13.26) then yields the result for $2<p \leq \infty$ as well.

Remark 13.1. Alternatively, we could have performed higher derivative estimates as in the proof of Proposition 10.2 and carried them along in the analysis to obtain a self-contained argument involving only Sobolev estimates, for initial data in the smaller space $L^{1} \cap H^{d}$. This would require the same regularity $f \in C^{\left[\frac{d}{2}\right]+5}$ as the $\mathrm{KK}$-type argument used in previous sections; by contrast, the present argument requires only $f \in C^{2}$. 
13.3. Relation between $[\mathrm{KK}]$ and $[\mathrm{Z1}]$ analyses. We conclude by a brief further discussion of the relation between [KK] and [Z1] approaches, in light of the above calculations. Clearly, the pointwise resolvent kernel bounds of [Z1] were not the essential point (we have not used them here), but rather the consequent $L^{1} \rightarrow L^{p}$ resolvent bounds and the improved bounds for $\rho$-forcing. These bounds are shared, central features of both the [Z1] and $\mathrm{KK}$ analyses. (However, note: The $\mathrm{KK}$ resolvent analysis is purely one-dimensional, so it does not address the important technical issue of glancing modes; indeed, it is not immediately clear that their method of obtaining resolvent bounds can be generalized to higher dimensions.)

The main difference, then, is in the endgame by which the resolvent bounds are converted to nonlinear estimates. The [KK] approach could be described as "hyperbolic," since it uses an iteration scheme very similar to that of the inviscid case. In particular, the integration of $\lambda$ along the imaginary axis does not reveal the effects of diffusion. As we have demonstrated here, this scheme is applicable for general (nonzero mass) initial data only for dimensions greater than or equal to three. The endgame of [Z1] could be described rather as "parabolic": integration on the parabolic contour $\Gamma\left(\xi^{\prime}\right)$ reveals an additional temporal decay due to diffusion that is essential to the proof of nonlinear stability in dimensions less than or equal to two. (Note: In dimension one, somewhat further care is needed; specifically, translation of the shock must be projected out [Z2, MaZ1, MaZ2, MaZ3, MaZ4. MaZ5, Z3, HZ, Ra.)

With regard to the small viscosity limit, we point out that both choices of contour are consistent with the standard hyperbolic analysis, since the curvature of the parabolic contour is taken proportional to viscosity. A very interesting direction in the small viscosity theory would be to investigate whether there is any advantage to working on such parabolic contours to take into account the beneficial effects of diffusion. It is not clear, however, how to incorporate this into the argument structure of, e.g., [MZ1 GMWZ1].

\section{REFERENCES}

[Co] Coppel, W. A., Stability and asymptotic behavior of differential equations, D.C. Heath, Boston. MR0190463 (32:7875)

[CP] Chazarain, J. and Piriou, A., Introduction to the Theory of Linear Partial Differential Equations, North Holland, Amsterdam, 1982. MR0678605 (83j:35001)

[FM] Francheteau, J. and Métivier, G., Existence de chocs faibles pour des systèmes quasilinéaires hyperboliques multidimensionnels, C.R.AC.Sc. Paris, 327 Série I, 1998, 725728. MR1660029 (99g:35081)

[FL] Freistühler, H. and Liu, T.-P., Nonlinear stability of overcompressive shock waves in a rotationally invariant system of viscous conservation laws, Commun. Math. Phys. 153, 1993, 147-158. MR1213739 (94f:35084)

[FS] Freistühler, H. and Szmolyan, P., Spectral stability of small shock waves, I, Arch. Rat. Mech. Analysis, 164, 2002, 287-309. MR1933630 (2003j:35273)

[FZ] Freistühler, H. and Zumbrun, K., Examples of unstable viscous shock waves. Unpublished note, Institut für Mathematik, RWTH Aachen, February 1998.

[GZ] Gardner, R. and Zumbrun, K., The gap lemma and geometric criteria instability of viscous shock profiles, Comm. Pure Appl. Math. 1998, 797-855. MR.1617251 (99c:35152)

[Go1] Goodman, J., Nonlinear asymptotic stability of viscous shock profiles for conservation laws, Arch. Rational Mech. Analysis 95, 1986, 325-344. MR0853782 (88b:35127)

[Go2] Goodman, J., Remarks on the stability of viscous shock waves, in: Viscous profiles and numerical methods for shock waves (Raleigh, NC, 1990), 66-72, SIAM, Philadelphia, PA, 1991. MR1142641 
[Go3] Goodman, J., Stability of viscous scalar shock fronts in several dimensions, Trans. Amer. Math. Soc. 311, 1989, no. 2, 683-695. MR.0978372 (89j:35059)

[GM] Goodman, J. and Miller, J. R., Long-time behavior of scalar viscous shock fronts in two dimensions, J. Dynam. Differential Equations 11, 1999, no. 2, 255-277. MR1695245 (2000e:35147)

[GMWZ1] Guès, O., Métivier, G., Williams, M., and Zumbrun, K., Multidimensional viscous shocks II: the small viscosity limit, Comm. Pure Appl. Math., 57, 2004, 141-218. MR2012648

[GMWZ2] Guès, O., Métivier, G., Williams, M., and Zumbrun, K., Existence and stability of multidimensional shock fronts in the vanishing viscosity limit, to appear in Arch. Rational Mech. Anal.

[GMWZ3] Guès, O., Métivier, G., Williams, M., and Zumbrun, K., Navier-Stokes regularization of multidimensional Euler shocks, in preparation.

[GMWZ4] Guès, O., Métivier, G., Williams, M., and Zumbrun, K., Stability of viscous shock waves of symmetric systems with variable multiplicities, in preparation.

[GW] Guès, O. and Williams, M., Curved shocks as viscous limits: a boundary problem approach, Indiana Univ. Math. J. 51, 2002, 421-450. MR1909296 (2003h:35166)

[HoZ1] Hoff, D. and Zumbrun, K., Pointwise decay estimates for multidimensional NavierStokes diffusion waves, Z. Angew. Math. Phys. 48, 1997, 597-614. MR1471469 (98i:35145)

[HoZ2] Hoff, D. and Zumbrun, K., Pointwise Green's function bounds for multi-dimensional scalar viscous shock fronts. J. Differential Equations 183, 2002, 368-408. MR1919784 (2003f:35194)

[HoZ3] Hoff, D. and Zumbrun, K., Asymptotic behavior of multi-dimensional scalar viscous shock fronts. Indiana Univ. Math. J. 49, 2000, 427-474. MR1793680 (2001j:35195)

[H1] Howard, P., Pointwise estimates on the Green's function for a scalar linear convectiondiffusion equation, J. Differential Equations 155, 1999, 327-367. MR1698558 (2000e:35077)

[H2] Howard, P., Pointwise Green's function approach to stability for scalar conservation laws, Comm. Pure Appl. Math. 52, 1999, 1295-1313. MR1699970 (2000f:35093)

[HZ] Howard, P. and Zumbrun, K., Stability of undercompressive shock waves, Preprint 2004.

[K1] Kapitula, T., Stability of weak shocks in $\lambda-\omega$ systems, Indiana Univ. Math. J. 40, 1991, no. 4, 1193-1219. MR1142711 (93d:35073)

[K2] Kapitula, T., On the stability of travelling waves in weighted $L^{\infty}$ spaces, J. Diff. Eqs. 112, 1994, no. 1, 179-215. MF.1287557 (95h:35107)

[KS] Kapitula, T. and Sandstede, B., Stability of bright solitary-wave solutions to perturbed nonlinear Schödinger equations, Phys. D 124, 1998, no. 1-3, 58-103. MR1662530 (99h:35199)

[KMN] Kawashima, S., Matsumura, A., and Nishihara, K., Asymptotic behavior of solutions for the equations of a viscous heat-conductive gas, Proc. Japan Acad. Ser. A Math. Sci. 62, 1986, no. 7, 249-252. MR0868811 (87k:35036)

[K] Kreiss, H.-O., Initial boundary value problems for hyperbolic systems, Comm. Pure Appl. Math. 23, 1970, 277-298. MR0437941 (55:10862)

[KK] Kreiss, G. and Kreiss, H.-O., Stability of systems of viscous conservation laws, Comm. Pure Appl. Math. 50, 1998, 1397-1424. MR1639151 (2000c:35156)

[KKP] Kreiss, G., Kreiss, H.-O., and Petersson, N., On the convergence to steady state of solutions of nonlinear hyperbolic-parabolic problems, SIAM J. Num. Analysis, 31, 1994, 1577-1604. MR 1302676 (95h:35028)

[La] Lax, P. D., Hyperbolic systems of conservation laws and the mathematical theory of shock waves, Conf. Board of the Mathematical Sciences Regional Conference Series in Applied Mathematics, No. 11, Society for Industrial and Applied Mathematics, Philadelphia, PA, 1973, v + 48 pp. MR0350216 (50:2709)

[L1] Liu, T.-P., Nonlinear stability of shock waves for viscous conservation laws, Memoirs Amer. Math. Soc., 56, 1985, no. 328. MR0791863|(87a:35127)

[L2] Liu, T.-P., Pointwise convergence to shock waves for viscous conservation laws, Comm. Pure Appl. Math. 50, 1997, no. 11, 1113-1182. MR1470318 (98j:35121) 
[LX] Liu, T.-P. and Xin, Z., Stability of viscous shock waves associated with a system of non-strictly hyperbolic conservation laws, Comm. Pure Appl. Math. 45, 1992, no. 4, 361-388. MR.1161537 (93k:35170)

[LZe] Liu, T.-P. and Zeng, Y., Large time behavior of solutions for general quasilinear hyperbolic-parabolic systems of conservation laws, AMS Memoirs 599, 1997. MR.1357824 (97g:35107)

[LZ1] Liu, T.-P. and Zumbrun, K., Nonlinear stability of an undercompressive shock for complex Burgers equation, Comm. Math. Phys. 168, 1995, no. 1, 163-186. MR1324394 (96f:35109)

[LZ2] Liu, T.-P. and Zumbrun, K., On nonlinear stability of general undercompressive viscous shock waves, Comm. Math. Phys. 174, 1995, no. 2, 319-345. MR1362168 (97g:35110)

[M1] Majda, A., The stability of multidimensional shock fronts, Mem. Amer. Math. Soc. No. 275, AMS, Providence, 1983. MR0683422 (84e:35100)

[M2] Majda, A., The existence of multidimensional shock fronts, Mem. Amer. Math. Soc. No. 281, AMS, Providence, 1983. MR0699241 (85f:35139)

[MaZ1] Mascia, C. and Zumbrun, K., Pointwise Green's function bounds and stability of relaxation shocks, Indiana Univ. Math. J. 51, 2002, no. 4, 773-904. MR1947862 (2003k:35151)

[MaZ2] Mascia, C. and Zumbrun, K., Stability of small-amplitude shock profiles of symmetric hyperbolic-parabolic systems, Comm. Pure Appl. Math. 57, 2004, no. 7, 841-876. MR.2044067

[MaZ3] Mascia, C. and Zumbrun, K., Pointwise Green's function bounds for shock profiles with degenerate viscosity, Arch. Rational Mech. Anal. 169, 2003, no. 3, 177-263. MR2004135 (2004h:35137)

[MaZ4] Mascia, C. and Zumbrun, K., Stability of large-amplitude shock profiles of hyperbolicparabolic systems, Arch. Rational Mech. Anal. 172, 2004, no. 1, 93-131. MR2048568

[MaZ5] Mascia, C. and Zumbrun, K., Stability of large-amplitude shock profiles for general relaxation systems, Preprint 2003.

[MN] Matsumura, A. and Nishihara, K., On the stability of travelling wave solutions of a one-dimensional model system for compressible viscous gas, Japan J. Appl. Math. 2, 1985, no. 1, 17-25. MR0839317 (87j:35335a)

[Met1] Métivier, G., Problemes mixtes nonlineaires et stabilite des chocs multidimensionnels, Sem. Bourbaki 671, 1986, 37-53. MR0936847 (89f:35131)

[Met2] Métivier, G., Stability of multidimensional shocks, Advances in the theory of shock waves, Progress in Nonlinear PDE, 47, Birkhäuser, Boston, 2001. MR1842775 (2002m:35148)

[MZ1] Métivier, G. and Zumbrun, K., Large viscous boundary layers for noncharacteristic nonlinear hyperbolic problems, to appear in Memoirs of the Amer. Math. Soc., available at http://www.maths.univ-rennes1.fr/ metivier/preprints.html

[MZ2] Métivier, G. and Zumbrun, K., Hyperbolic boundary value problems for symmetric systems with variable multiplicities, to appear in J. Diff. Eq.; Preprint 2003.

[PZ] Plaza, R. and Zumbrun, K., An Evans function approach to spectral stability of smallamplitude shock profiles, J. Discrete and Continuous Dynamical Systems 10, 2004, no. 4, 885-924. MR.2073940

[Ra] Raoofi, M., $L^{1}$-asymptotic behavior of perturbed viscous shock profiles, Thesis, Indiana University, 2004.

[R] Rousset, F., Viscous approximation of strong shocks of systems of conservation laws. SIAM J. Math. Anal. 35, 2003, no. 2, 492-519. MF2001110 (2004i:35218)

[SX] Szepessy, A. and Xin, Z., Nonlinear stability of viscous shock waves, Arch. Rat. Mech. Analysis, 122, 1993, 53-103. MR1207241 (93m:35125)

[Z1] Zumbrun, K., Multidimensional stability of planar viscous shock waves, Advances in the theory of shock waves, 304-516. Progress in Nonlinear PDE, 47, Birkhäuser, Boston, 2001. MR.1842778 (2002k:35200)

[Z2] Zumbrun, K., Refined Wave-tracking and Nonlinear Stability of Viscous Lax Shocks, Methods Appl. Anal. 7, 2000, 747-768.

[Z3] Zumbrun, K., Stability of large-amplitude shock waves for compressible Navier-Stokes equations, to appear in Handbook of Mathematical Fluid Mechanics, Elsevier, 2004. 
[Z4] Zumbrun, K., Planar stability criteria for viscous shock waves of systems with real viscosity, to appear in CIME lecture notes series; Preprint 2004.

[ZH] Zumbrun, K. and Howard, P., Pointwise semigroup methods and stability of viscous shock waves, Indiana Univ. Math. J. 47, 1998, 741-871. MR.1665788 (99m:35157)

[ZS] Zumbrun, K. and Serre, D., Viscous and inviscid stability of multidimensional planar shock fronts, Indiana Univ. Math. J. 48, 1999, 937-992. MR.1736972 (2001h:35122)

LatP, Université de Provence, 39 rue Joliot-Curie, 13453 Marseille, France

E-mail address: gues@cmi.univ-mrs.fr

maB, Université de Bordeaux 1, 351 cours de la Libération, 33405 Talence Cedex, FRANCE

E-mail address: Guy.Metivier@math.u-bordeaux.fr

University of North Carolina, Department of Mathematics, CB 3250, Phillips Hall, Chapel Hill, NC 27599

E-mail address: williams@email.unc.edu

Indiana University, Department of Mathematics, Rawles Hall, Bloomington, IN 47405

E-mail address: kzumbrun@indiana.edu 